\title{
KRONECKER WEBS, BIHAMILTONIAN STRUCTURES, AND THE METHOD OF ARGUMENT TRANSLATION
}

\author{
ILYA ZAKHAREVICH
}

\begin{abstract}
We show that manifolds which parameterize values of first integrals of integrable finite-dimensional bihamiltonian systems carry a geometric structure which we call a Kronecker web. We describe two opposite-direction functors between Kronecker webs and integrable bihamiltonian structures, one is left inverse to the other. Conjecturally, these two functors are mutually inverse (for "small" open subsets of the manifolds in question).

The conjecture above is proven here when the bihamiltonian structure allows an anti-involution of a particular form. This implies the conjecture of [11] that on a dense open subset the bihamiltonian structure on $\mathfrak{g}^{*}$ is flat if $\mathfrak{g}$ is semisimple, or if $\mathfrak{g}=\mathfrak{G} \ltimes \operatorname{ad}_{\mathfrak{G}}$ and $\mathfrak{G}$ is semisimple, and for some other Lie algebras of mappings.
\end{abstract}

\section{Contents}

0. Basic notions 2

1. Introduction 6

2. Linear relations and pencils 12

3. Kronecker relations 14

4. Kronecker webs $\quad 15$

5. Pairs of skew-symmetric forms 22

6. Micro-Kronecker bihamiltonian structures 23

7. Lattice of kernels 26

8. Lagrangian foliations 27

9. Two functors 29

10. Conjecture on classification 31

11. Anti-involutions 32

12. Real and complex bihamiltonian structures 35

13. Endomorphisms of bihamiltonian structures and the principal theorem 36

14. Method of argument translation 38

15. Appendix on Kronecker decompositions 48

References $\quad 53$

Date: August 1999 (Revision III: March 2000) Archived as math.SG/9908034 Printed: September 16, 2021. 


\section{BASIC NOTIONS}

We postpone the informal discussion of what is done in this paper until Section 1, and start with introduction of notations and conventions used throughout this text. People familiar with basic notions and terminology of bihamiltonian geometry may jump directly to Section 1, looking up Examples 0.9 and 0.10 on the "when needed" basis.

Many results of this paper may be stated in greater generality, but for simplicity we assume that all the vector spaces we consider here are finite-dimensional ${ }^{1}$ vector spaces over a field $\mathbb{K}$ which is either $\mathbb{R}$ or $\mathbb{C}$. A manifold is a $C^{\infty}$-manifold or a realanalytic manifold in the case $\mathbb{K}=\mathbb{R}$, and an analytic manifold in the case $\mathbb{K}=\mathbb{C}$. We use the word smooth to mean $C^{\infty}$-smooth, real-analytic, or complex-analytic correspondingly.

For a vector space $V$ over $\mathbb{K}$ denote by $V^{*}$ the space of $\mathbb{K}$-linear functionals on $V$. Note that throughout this paper we do not consider semilinear functionals or Hermitian forms on complex vector spaces.

We start by recalling some basic notions and notations of Poisson geometry (see $[2,16,3])$. In what follows if $f$ is a function or a tensor field on $M,\left.f\right|_{m}$ denotes the value of $f$ at $m \in M$.

Remark 0.1. Throughout the paper we use standard idioms of differential geometry. Say the phrase "at generic points" means "at points of an appropriate open dense subset". Similarly, a "small open subset" is used instead of "an appropriate neighborhood of any given point". A local isomorphism between two geometric structures on $M$ and $M^{\prime}$ is an isomorphism of a neighborhood of a given point on $M$ with a neighborhood of a given point on $M^{\prime}$. Two geometric structures on $M$ and on $M^{\prime}$ are locally isomorphic if for any $m \in M$ and $m^{\prime} \in M^{\prime}$ there is a local isomorphism which sends $m$ to $m^{\prime}$.

Definition 0.2. A bracket on a manifold $M$ is a $\mathbb{K}$-bilinear skew-symmetric mapping which sends a pair of smooth functions ${ }^{2} f, g$ on $M$ to a smooth function $\{f, g\}$ on $M$. This mapping is required to satisfy the Leibniz identity $\{f, g h\}=g\{f, h\}+h\{f, g\}$. A bracket is Poisson if it satisfies the Jacobi identity $\{f,\{g, h\}\}=\{\{f, g\}, h\}+$ $\{g,\{f, h\}\}$ (thus defines a structure of a Lie algebra on functions on $M$ ).

A Poisson structure is a manifold $M$ equipped with a Poisson bracket.

Remark 0.3. Leibniz identity implies $\left.\{f, g\}\right|_{m}=0$ if $f$ has a zero of second order at $m \in M$, or if $f \equiv$ const. Thus a bracket is uniquely determined by describing the functions $\left\{f_{i}, f_{j}\right\}$; here $\left\{f_{i}\right\}_{i \in I}$ is an arbitrary collection of functions on $M$ which separates points of $M$. Here we say that a collection $\left\{f_{i}\right\}_{i \in I}$ of smooth functions on

\footnotetext{
${ }^{1}$ With obvious exceptions of vector spaces of functions on manifolds.

${ }^{2}$ In the complex-analytic case one should consider functions on open subsets $U \subset M$ and require that the brackets on these subsets are compatible on intersections.
} 
$M$ separates points of $M$ if for any $m \in M$ the collection $\left\{\left.d f_{i}\right|_{m}\right\}_{i \in I}$ of vectors in $\mathcal{T}_{m}^{*} M$ spans $\mathcal{T}_{m}^{*} M$ as a vector space.

Definition 0.4. Consider a bracket $\{$,$\} on a manifold M$. The associated bivector ${ }^{3}$ field $\eta$ is the section of $\Lambda^{2} \mathcal{T} M$ given by $\left.\{f, g\}\right|_{m}=\left\langle\left.\eta\right|_{m},\left.d f \wedge d g\right|_{m}\right\rangle, m \in M$; here $\langle$,$\rangle denotes the canonical pairing between \Lambda^{2} \mathcal{T}_{m} M$ and $\Omega_{m}^{2} M$.

Given $m_{0} \in M$, the associated pairing (,) in $\mathcal{T}_{m_{0}}^{*} M$ is defined as $(\alpha, \beta)=\left.\{f, g\}\right|_{m_{0}}$ if $\alpha=\left.d f\right|_{m_{0}}, \beta=\left.d g\right|_{m_{0}}$.

Obviously, the associated bivector field uniquely determines the bracket and visa versa. The associated pairing is a skew-symmetric bilinear pairing.

Definition 0.5. Given a skew-symmetric bilinear form (,) in a vector space $V$, put $\operatorname{Ker}(,) \stackrel{\text { def }}{=}\left\{v \in V \mid\left(v, v^{\prime}\right)=0 \forall v^{\prime} \in V\right\}$, and call $\operatorname{dim} \operatorname{Ker}($,$) the corank of ($,$) . The$ rank of (,) is $\operatorname{dim} V-\operatorname{dim} \operatorname{Ker}($,$) .$

The rank of the bracket $\{$,$\} at m \in M$ is $r$ if the associated skew-symmetric bilinear pairing on $\mathcal{T}_{m}^{*} M$ has rank $r$. In this case the corank of the bracket is $\operatorname{dim} M-r$.

A bracket has a constant (co)rank if its rank does not depend on the point $m \in M$. A Poisson bracket is symplectic if the corank is constant and equal to 0 .

The associated tensor field $\eta$ of a bracket on $M$ can be considered as a mapping $\mathrm{H}: \mathcal{T}^{*} M \rightarrow \mathcal{T} M$ (the Hamiltonian mapping of a bracket). If the bracket is symplectic, this mapping is invertible, and the inverse mapping $\mathrm{H}^{-1}: \mathcal{T} M \rightarrow \mathcal{T}^{*} M$ can be considered as a bilinear pairing on $\mathcal{T} M$, or as a tensor field. This tensor field is a section $\omega$ of $\Omega^{2} M$ of corank 0 , called the symplectic 2 -form of the symplectic bracket. In local coordinates the tensors $\eta$ and $\omega$ are given by mutually inverse matrices.

In the other direction, given a section $\omega$ of $\Omega^{2} M$ of corank 0 , setting $\eta=\omega^{-1}$ gives a bracket on $M$. It is easy to check that $\eta$ is Poisson iff ${ }^{4} d \omega=0$.

Example 0.6. Given a manifold $N$, put $M=\mathcal{T}^{*} N$, and let $\pi: M \rightarrow N$ be the natural projection. Given $m \in M$, one can write $m=(n, \nu), n \in N, \nu \in \mathcal{T}_{n}^{*} N$. Apply $\pi^{*}: \mathcal{T}_{n}^{*} N \rightarrow \mathcal{T}_{m}^{*} M$ to $\nu$, and note that $\pi^{*} \nu$ is an element of $\mathcal{T}_{m}^{*} M$ which depends on $m$ only. One can write $\pi^{*} \nu=\alpha(m)$; here $\alpha$ is a canonically defined section of $\Omega^{1} M$.

Local coordinates $\left(n_{1}, \ldots, n_{d}\right)$ on $N$ define local coordinates $\left(n_{1}, \ldots, n_{d}, \nu_{1}, \ldots, \nu_{d}\right)$ on $\mathcal{T}^{*} N$. In these coordinates $\alpha=\sum_{i} \nu_{i} d n_{i}$. Taking $\omega=d \alpha$, one obviously gets $d \omega=0$. In local coordinates $\omega=\sum_{i} d \nu_{i} \wedge d n_{i}$, hence $\omega$ is of corank 0 , consequently defines a (symplectic) Poisson structure $\eta$ on $M=\mathcal{T}^{*} N$.

Recall that any symplectic Poisson structure is locally isomorphic to the structure of Example 0.6.

\footnotetext{
${ }^{3} \mathrm{~A}$ bivector field is a skew-symmetric contravariant tensor of valence 2 .

${ }^{4}$ This condition is linear in $\omega$, as opposed to the quadratic condition (of Jacobi identity) on $\eta$. This linearity makes it much easier to study symplectic Poisson brackets.
} 
Definition 0.7. Call two Poisson brackets $\{,\}_{1}$ and $\{,\}_{2}$ on $M$ compatible if the bracket $\lambda_{1}\{,\}_{1}+\lambda_{2}\{,\}_{2}$ is Poisson for any $\lambda_{1}, \lambda_{2}$.

A bihamiltonian structure is a manifold $M$ with a pair of compatible Poisson brackets.

Given that the Jacobi identity is "quadratic" in $\{$,$\} , one can show that if one$ linear combination $\lambda_{1}\{,\}_{1}+\lambda_{2}\{,\}_{2}$ of two Poisson brackets is Poisson, and $\lambda_{1} \neq 0$, $\lambda_{2} \neq 0$, then any linear combination $\lambda_{1}\{,\}_{1}+\lambda_{2}\{,\}_{2}$ is Poisson. Even if $M$ is a $C^{\infty}$-manifold, the coefficients $\lambda_{1}, \lambda_{2}$ may be taken to be complex numbers. Indeed, if $M$ is a $C^{\infty}$-manifold with a bracket, one may consider the extension of the bracket to the $\mathbb{C}$-vector space of complex-valued functions on $M$. In this case $\lambda_{1}\{,\}_{1}+\lambda_{2}\{,\}_{2}$ is well-defined even for complex values of $\lambda_{1}, \lambda_{2}$. By the remarks above, complex linear combinations of brackets of a bihamiltonian structure are also Poisson. In what follows we can always consider brackets as acting on the spaces of complex-valued functions.

Given a pair of brackets $\{,\}_{1}$ and $\{,\}_{2}$, one obtains two bivector fields $\eta_{1}, \eta_{2}$. Analogously, one obtains two skew-symmetric bilinear pairings $(,)_{1},(,)_{2}$ on $\mathcal{T}_{m}^{*} M$, so that $(\alpha, \beta)_{j}=\left.\{f, g\}_{j}\right|_{m}$ if $\alpha=\left.d f\right|_{m}, \beta=\left.d g\right|_{m}, j=1,2$.

Definition 0.8. A Poisson structure $\{$,$\} on a vector space V$ is translation-invariant if for any parallel translation $\mathfrak{T}: V \rightarrow V$ and any two functions $f, g$ on $V$ one has $\mathfrak{T}^{*}\{f, g\}=\left\{\mathfrak{T}^{*} f, \mathfrak{T}^{*} g\right\}$. A bihamiltonian structure on $V$ is translation-invariant if both Poisson brackets are translation-invariant.

A bihamiltonian structure on a manifold $M$ is flat if it is locally isomorphic to a translation-invariant bihamiltonian structure. In other words, for any $m \in M$ there is a neighborhood $U \ni m$ such that the restriction of the bihamiltonian structure to $U$ is isomorphic to the restriction of an appropriate translation-invariant bihamiltonian structure to an appropriate open subset.

The tensor field $\eta$ of a translation-invariant Poisson bracket on $V$ has constant coefficients in any vector-space coordinate system on $V$. For us one particular example of a translation-invariant bihamiltonian structure is of special interest.

Example 0.9. Consider a vector space $V$ with coordinates $x_{0}, \ldots, x_{2 k-2}$. Define Poisson brackets $\{,\}_{1}$ and $\{,\}_{2}$ on the coordinate functions by

$$
\left\{x_{2 l}, x_{2 l+1}\right\}_{1}=1, \quad\left\{x_{2 l+1}, x_{2 l+2}\right\}_{2}=1, \quad 0 \leq l \leq k-2,
$$

and setting any other bracket of the coordinate functions $x_{0}, \ldots, x_{2 k-2}$ to be zero. This pair of brackets is in fact a translation-invariant bihamiltonian structure.

The following example is the simplest of the classical examples of bihamiltonian structures arising in the theory of integrable systems.

Example 0.10. Given a Lie algebra $\mathfrak{g}$ and an element $c_{1} \in \mathfrak{g}^{*}$, define a bihamiltonian structure on $\mathfrak{g}^{*}$ as in $[25,4]$. An element $X \in \mathfrak{g}$ defines a linear function $f_{X}$ on $\mathfrak{g}^{*}$. 
Due to Remark 0.3, to define a bihamiltonian structure on $\mathfrak{g}^{*}$ it is enough to describe the brackets $\left\{f_{X}, f_{Y}\right\}_{j}, j=1,2, X, Y \in \mathfrak{g}$.

Let $\left\{f_{X}, f_{Y}\right\}_{1}$ be the constant function on $\mathfrak{g}^{*}$ and $\left\{f_{X}, f_{Y}\right\}_{2}$ be the linear function on $\mathfrak{g}^{*}$ given by the formulae

$$
\left\{f_{X}, f_{Y}\right\}_{1}=f_{[X, Y]}\left(c_{1}\right), \quad\left\{f_{X}, f_{Y}\right\}_{2}=f_{[X, Y]} .
$$

The bracket $\{,\}_{2}$ is the natural Lie-Kirillov-Kostant-Souriau Poisson bracket on $\mathfrak{g}^{*}$. The bracket $\{,\}_{1}$ is translation-invariant. The bracket $\{,\}_{2}$ is translation-invariant only if $\mathfrak{g}$ is abelian (and then $\{,\}_{1,2}$ vanish).

In fact, instead of taking $c_{1} \in \mathfrak{g}^{*}$ one can consider any 2-cocycle $c_{2} \in \Lambda^{2} \mathfrak{g}^{*}$, and define $\left\{f_{X}, f_{Y}\right\}_{1}=c_{2}(X, Y)$. The definition above is recovered if one puts $c_{2}=\partial c_{1}$. If $H^{2}(\mathfrak{g}, \mathbb{C})=0$ then these two versions of the definition are equivalent.

Definition 0.11. A smooth function $F$ on a manifold $M$ with a Poisson bracket $\{$, is a Casimir function if $\{F, f\}=0$ for any smooth function $f$ on $M$.

Obviously, any function $\varphi\left(F_{1}, F_{2}, \ldots, F_{k}\right)$ of several Casimir functions is again Casimir.

Definition 0.12. A collection of smooth functions $F_{1}, \ldots, F_{r}$ on $M$ is dependent if $\varphi\left(F_{1}, \ldots F_{r}\right) \equiv 0$ for an appropriate smooth function $\varphi$ such that $\varphi \not \equiv 0$ on any open subset of $\mathbb{K}^{r}$.

Remark 0.13. Consider a manifold $M$ with a Poisson bracket $\{$,$\} . The local classi-$ fication of Poisson structures of constant rank [17, 37] shows that for an arbitrary Poisson bracket there is an open (and in interesting cases dense) subset $U \subset M$ and $k \in \mathbb{Z}_{\geq 0}$ such that on $U$ there are $k$ independent Casimir functions $F_{1}, \ldots, F_{k}$, and any Casimir function on $U$ may be written as a function of $F_{1}, \ldots, F_{k}$ (we do not exclude the case $k=0$ ). The common level sets $F_{1}=C_{1}, \ldots, F_{k}=C_{k}$ form an invariantly defined foliation on $U$, which is called the symplectic foliation. Moreover, locally one can construct additional functions $n_{i}, \nu_{i}, i=1, \ldots, d$, on $U$ such that $\left\{n_{i}, n_{j}\right\}=\left\{\nu_{i}, \nu_{j}\right\}=0,\left\{n_{i}, \nu_{j}\right\}=\delta_{i j}$, and the functions $F_{\bullet}, n_{\bullet}, \nu_{\bullet}$ form a coordinate system on $U$.

This shows that any Poisson structure of constant rank is flat, i.e., locally isomorphic to a translation-invariant Poisson structure. Consequently, any analytic Poisson structure is flat on an open dense subset. Moreover, the leaves of the symplectic foliation of such Poisson structures can be simultaneously equipped with coordinates as in Example 0.6.

Definition 0.14. Consider a foliation $\mathcal{F}$ on $B$. Define the tangent bundle $\mathcal{T} \mathcal{F}$ to $\mathcal{F}$ to be the vector subbundle $E$ of $\mathcal{T} B$ such that $E_{b}$ coincides with $\mathcal{T}_{b} L_{b}$ for any $b \in B$; here $L_{b}$ is the leaf of $\mathcal{F}$ which passes through $b$. Let the normal bundle $\mathcal{N} \mathcal{F} \subset \mathcal{T}^{*} B$ to $\mathcal{F}$ be the orthogonal complement of $\mathcal{T} \mathcal{F}$, and the cotangent bundle $\mathcal{T}^{*} \mathcal{F}$ to $\mathcal{F}$ be the vector bundle dual to $\mathcal{T} \mathcal{F}$. 
The total space of the bundle $\mathcal{T}^{*} \mathcal{F}$ is a union of the total spaces of the cotangent bundles $\mathcal{T}^{*} L$ of the leaves $L$ of the foliation. Since each $\mathcal{T}^{*} L$ carries a natural symplectic Poisson structure (Example 0.6), one obtains

Proposition 0.15. The total space of the cotangent bundle to a foliation carries a natural Poisson structure of constant rank.

Moreover, the foliation on $\mathcal{T}^{*} \mathcal{F}$ specified above is the symplectic foliation of this Poisson structure. Due to Remark 0.13, any Poisson structure of constant rank is locally isomorphic to the Poisson structure on $\mathcal{T}^{*} \mathcal{F}$ of a foliation $\mathcal{F}$ with the appropriate dimension and rank.

\section{INTRODUCTION}

Among other approaches to integrable systems ${ }^{5}$ the so-called bihamiltonian approach is especially interesting from the geometric point of view. In this approach all the properties of an integrable bihamiltonian system are deduced from the bihamiltonian structure on the phase manifold ${ }^{6}$. (The principal tool for this deduction is Lenard scheme, which is outside of the scope of this paper, see $[20,21,10,8,18,11]$.) Since the structure of a bihamiltonian system is nothing more than a pair of tensor fields satisfying some invariantly-defined conditions, this approach puts the integrable system into the standard framework of differential geometry.

A powerful approach to study a particular object of differential geometry is to classify all possible objects up to isomorphism, and to describe the automorphisms of every object. After this what remains is to describe the position of the given object inside the classification. For example, in the case of symplectic structures or Poisson structures of constant rank, the local classification is "trivial": locally there are only some discrete parameters, thus any structure is locally isomorphic to one from a finite list (for a given dimension). This reduces all questions on geometry of symplectic manifolds to questions of global nature. Similarly, all questions on geometry of Poisson manifolds are reduced to questions of global nature, and to questions related to subsets where the rank drops.

Classification in general position. Starting from the end of the $80 \mathrm{~s}$, there were many results on local classifications of bihamiltonian structures "in general position". The spirit of these classification efforts follows the results on classification of the corresponding linearized problem: classification of pairs of skew-symmetric bilinear forms in a vector space. The pairs in general position behave differently depending on the dimension: an even-dimensional pair in general position is a direct

\footnotetext{
${ }^{5}$ An integrable system is a notion which is used in different senses in different situations. For the sake of this introduction, one can understand it as a Liouville integrable system. In other words, it is a system of ODE which can be solved explicitly, and such that the solutions demonstrate no mixing property: trajectories stay in closed submanifolds of "small" dimension.

${ }^{6}$ There is a widespread belief that most (or all) integrable systems which arise in problems of mathematical physics allow a natural bihamiltonian structure.
} 
sums of 2-dimensional blocks, while an odd-dimensional pair in general position is an indecomposable block (a Kronecker block; for details see Section 5).

For a long time the case of general position on even-dimensional manifolds was considered to be the most relevant to the theory of integrable systems. This classification was done in $[33,22,23,24,14]$ (under different assumptions). The case of general position in the odd-dimensional case was analyzed in $[13,14,30,29,31,34,35]$. However, until recently these results had little direct impact on the theory of integrable systems, since it was not known which classical integrable systems are subject to these conditions of general position. To answer this question, one would need to classify a linearized bihamiltonian structure in a generic point of a particular classical integrable system.

Applications to integrable systems. It was a very old idea of I. M. Gelfand that a key to the bihamiltonian geometry might be uncovered by studying the linearized bihamiltonian structure of the periodic KdV system. In the mid-80s (i.e., prior to all of the works mentioned above), the author started a joint work with I. M. Gelfand on this problem. This turned out to be a very hard problem of functional analysis, and the first results [12] were achieved only after several years of intensive work. However, the fruits of this work deserved the effort, as the results of $[12,15]$ were completely orthogonal to the paradigms of the time.

It turned out that the periodic KdV system should be considered as an infinitedimensional analogue of an odd-dimensional bihamiltonian structure in general position. The analogous finite-dimensional pairs of skew-symmetric forms are exactly the Kronecker blocks mentioned above, and the Lenard scheme mentioned above works for any such pair. It was this analogy which fueled us to investigate the geometry of bihamiltonian structures linearizations of which at any point are Kronecker blocks; this we carried out in $[13,14]$.

Already at that time we knew an example of a classical bihamiltonian system which is subject to the classification above: the open Toda lattice (for definition see, for example, [7]). To some extent, Toda lattices are finite-dimensional analogues of the $\mathrm{KdV}$ system, so one could have expected that the periodic Toda lattice might have properties similar to a periodic KdV system. Unfortunately, the periodic Toda lattice is an even-dimensional bihamiltonian system, thus the direct analogy could not work. However, an open Toda lattice is an odd-dimensional bihamiltonian system, and it is relatively easy to check that the linearization at a point in general position is a Kronecker block.

Cases of non-general position. We had one example where Kronecker blocks played a vital role, but this was not enough to change a paradigm. What was needed was to have more examples, in particular, to investigate what happens with the periodic Toda lattice. Again, it took a lot of time until an understanding of the situation emerged in [11]. Near points in general position the periodic Toda lattice turned out to be locally isomorphic to a product of two copies of odd-dimensional bihamiltonian 
manifolds, each of them subject to the classification above. ${ }^{7}$ Additionally, we could demonstrate similar results for linearizations of other classical bihamiltonian systems: the linearizations break down into a direct sum of Kronecker blocks, i.e., into a direct sum of odd-dimensional components.

Flatness. These results show that Toda lattices are subject to the local classification of odd-dimensional bihamiltonian systems of $[13,14]$ (possibly after splitting into a direct product). Let us stress that there are infinitely many non-isomorphic bihamiltonian systems of these types (with parameters being several functions of two variables), thus it is meaningful to ask which system of the classification above is the open Toda lattice (or a factor of the periodic Toda lattice).

Recall that the simplest possible odd-dimensional bihamiltonian structure (in general position) is the structure of Example 0.9. One of the principal results of [11] is that the bihamiltonian structure of the open Toda lattice is locally isomorphic ${ }^{8}$ (on an open dense subset) to the structure of Example 0.9 (here $k$ is the number of "atoms" in the Toda lattice). Moreover, the bihamiltonian structure of the periodic Toda lattice is locally isomorphic (on an open dense subset) to a direct product of two copies of the structure of Example 0.9. (If the periodic Toda lattice has $n$ atoms, then one copy has $k=n$, another $k=1$.)

Extending this observation, one of the conjectures of [11] says that other integrable systems of mathematical physics are also locally isomorphic to a direct product of several copies of Example 0.9. In other words, these systems are flat on an open dense subsets. By the reasons which will be explained shortly, here we call this conjecture the metaconjecture.

There are similarities and differences of this metaconjecture with the result on flatness on an open dense subset of analytic Poisson manifolds. As in the Poisson case, this metaconjecture reduces questions on geometry of these integrable systems to two questions: the description of the behaviour at the points outside the dense open subsets mentioned above (points where the bihamiltonian structure degenerates), and the description of the gluing process. The question of local geometry in generic points mostly disappear: the system is locally isomorphic to one from a finite list $^{9}$ (for a fixed dimension).

The principal difference when comparing with the Poisson case is that - as pointed out already - in general bihamiltonian geometry is not locally flat. The metaconjecture above is a selection principle ${ }^{10}$ : out of a huge variety of different integrable bihamiltonian systems the systems studied in mathematical physics fall into a very thin subclass of flat bihamiltonian systems.

\footnotetext{
${ }^{7}$ In particular, a linearization at a point of the periodic Toda lattice is not in general position.

${ }^{8}$ Contrast this with the property of having isomorphic linearizations which we discussed before.

${ }^{9}$ For example, due to the result above they are locally isomorphic to a direct product of several open Toda lattices.

${ }^{10}$ For example, any proof of this metaconjecture (if possible) would need to concentrate on the question why mathematical physicists study some systems and do not study some other systems.
} 
The paper [11] also lists some specializations of the metaconjecture above: it is conjectured that some particular bihamiltonian systems of mathematical physics are flat on an open dense subset. This list includes the full Toda lattice (for definition see [19]), the multi-dimensional Euler top (for definition see [26]), and the semisimple case of Example 0.10, and some other examples related to Toda lattices.

Here is the current status of these special-case conjectures: in addition to the already proven case of various Toda lattices ([11]), in this paper we establish the semisimple case of Example 0.10 (see Corollary 14.24). This provides several established examples of "classical" integrable bihamiltonian systems which locally look like a product of the structures of Example 0.9.

Integrability. The examples above show that Kronecker blocks play an important role in geometry of classical integrable systems. Compare this with another reference point: [4] formalized the notion of integrability of a bihamiltonian system under the name of completeness. This notion is very close to the property of being microKronecker ${ }^{11}$ that we introduce below, in Section 6: a bihamiltonian system is complete if linearizations at points of an open dense subset break into a direct sum of Kronecker blocks.

All of the examples above (as most of the other bihamiltonian systems of mathematical physics) happen to be integrable in this strict sense. In this informal introduction we always use the word integrable in the sense of being micro-Kronecker. A system is complete if it integrable on an open dense subset. This makes it very important to investigate the local geometry of arbitrary integrable bihamiltonian systems. This investigation is one of the principal targets of this paper.

Webs. We mentioned two kinds of bihamiltonian structures with relationship to Kronecker blocks: odd-dimensional bihamiltonian structures in general position, and micro-Kronecker (or integrable) bihamiltonian structures. The former ones are particular cases of the latter ones, the condition being of having exactly one Kronecker block. One of principal results of [13] is the introduction of a notion of Veronese web (which is a family of foliations with appropriate compatibility conditions). As [13] and [34, 35] show, odd-dimensional bihamiltonian structures in general position are locally classified by a Veronese web on a manifold of (approximately) half the dimension of the initial manifold.

In [28] (and independently - more generally, but in less detail - in [11]) the construction of the Veronese web was generalized to the case of structures which are integrable in the sense of [4]. This paper starts with introduction of a geometric structure of a Kronecker web, which is simultaneously a generalization of the construction of a Veronese web of higher codimension of [28] and a more structured variant of the construction of a web of [11]. Each Kronecker web has a rank, and the Veronese webs of [13] coincide with Kronecker webs of rank 1.

\footnotetext{
${ }^{11}$ Different faces of the relationship of Kronecker blocks with integrability were independently discovered in [28] and in [11].
} 
Though the definition of a Kronecker web has no similarity with the definition of Veronese webs, results of Sections 7 imply that Kronecker webs can also be described as families of foliations with appropriate compatibility conditions.

Two functors and the principal conjecture. Similarly to what was done in [13] in the case of rank 1, we show how to associate to any integrable bihamiltonian structure its Kronecker web, and show how to construct an integrable bihamiltonian structure from an arbitrary Kronecker web. Conjecturally (Conjecture 10.1), these functors are mutually inverse (as in the case of rank 1 , see $[13,34,35]$ ), but here we prove only that one is left inverse to another.

If these functors were mutually inverse, the question of local classification of integrable bihamiltonian structures would be reduced to the question of local classification of Kronecker webs. Analogously to Definition 0.8, one can define a translationinvariant Kronecker web, and a flat Kronecker web. In particular, to show that a given bihamiltonian structure is flat, it would be enough to show that its Kronecker web is flat ${ }^{12}$.

For example, in [28] it is shown that in the semisimple case of Example 0.10 the corresponding Kronecker web is flat on an open dense subset. Together with our construction of two functors the conjecture above would immediately show that the bihamiltonian structure of Example 0.9 is flat on an open dense subset. Another example, the (known) case of rank 1 of this conjecture is used in [11] to demonstrate flatness of Toda lattices.

Moreover, note that proofs of flatness of webs of particular bihamiltonian systems are very simple (see [28, 11], and Theorem 14.22). Thus the theory of Kronecker webs allows one to condense all the problems of geometric classification of bihamiltonian structures into a proof of Conjecture 10.1 (or an appropriate particular case of this conjecture).

Anti-involutions and Lie algebras. In this paper we prove only a very special case of Conjecture 10.1. Section 11 introduces a special subclass of bihamiltonian structures, structures which allow an anti-involution of a special form. In Section 13 we show that in the case of such structures Conjecture 10.1 holds (on a large open subset).

Section 14 uses this approach to show that the semisimple case of Example 0.10 is flat on an open dense subset. As already explained, the flatness of the Kronecker web is already proved in [28]. We use standard tools of the theory of semisimple Lie algebras to construct appropriate anti-involutions. This provides an ad hoc way to prove this particular case of Conjecture 10.1.

Conclusions. This paper presents several important steps on the road to understanding geometry of bihamiltonian systems of mathematical physics. We introduce a notion of a Kronecker web (Section 4), and of a micro-Kronecker bihamiltonian structure (Section 6). Given a Kronecker web $B$, there is a canonically defined vector

\footnotetext{
${ }^{12} \mathrm{As}[13,14,11]$ show, there are plenty of examples of non-flat Kronecker webs, even in the case of rank 1 .
} 
bundle $\Phi$ over $B$ such that the total space $\Phi(B)$ of this bundle carries a canonically defined micro-Kronecker bihamiltonian structure (Corollary 4.21). Given a microKronecker bihamiltonian structure on a manifold $M$, there is a canonically defined foliation $\mathcal{F}$ on $M$ (the action foliation) such that the local base $\mathcal{B}_{M}$ of this foliation has a canonically defined structure of a Kronecker web (Theorem 6.7). The leaves of the action foliation on $\Phi(B)$ are fibers of the projection $\Phi(B) \rightarrow B$; thus $\mathcal{B}_{\Phi(B)} \simeq B$. Moreover, this isomorphism is compatible with the Kronecker web structures on $\mathcal{B}_{\Phi(B)}$ and on $B$ (Proposition 9.1).

We conjecture that the "inverse" is also true, at least in the following sense. Given a point $m$ on a manifold $M$ with a micro-Kronecker bihamiltonian structure, there is a Kronecker structure on $\mathcal{B}_{M}$, thus a micro-Kronecker bihamiltonian structure on $\Phi\left(\mathcal{B}_{M}\right)$. We conjecture that there is a local isomorphism between bihamiltonian structures on a neighborhood of $m$ and a neighborhood of $(b, 0)$; here $b$ is the image of $m$ in $\mathcal{B}_{M}$, and $(b, 0)$ is the point on the 0 -section of $\Phi\left(\mathcal{B}_{M}\right)$ over $b$. This constitutes Conjecture 10.1. (As the results of [13] show, this local isomorphism cannot be canonical in non-trivial cases.) This conjecture would reduce local classification of bihamiltonian structures up to isomorphism to a local classification of Kronecker webs up to isomorphism.

We prove a particular case of Conjecture 10.1, the case when the bihamiltonian structure on $M$ allows an anti-involution of a special form (Section 13). We apply this knowledge to several important particular cases of Example 0.10: the cases of semisimple $\mathfrak{g}$, and cases when $\mathfrak{g}$ is a Lie algebra of mappings from a self-dual Artin scheme (i.e., a Gorenstein scheme) to a semisimple Lie algebra. We show that in these cases the bihamiltonian structure of Example 0.10 is locally isomorphic to a direct product of several copies of Example 0.9 (when restricted on a dense open subset of $\mathfrak{g}^{*}$. This establishes one of conjectures of [11].

We believe that the same anti-involution trick will also work with complete Toda lattice and the multi-dimensional Euler top. However, we do not know whether this approach would work with the open and periodic Toda lattices. (Recall that the proof of [11] used the established case of rank 1 of Conjecture 10.1.)

Section 12 shows that the complex variant of Conjecture 10.1 implies the realanalytic case as well. The appendix (Section 15) describes which geometric structures one can invariantly associate to a Kronecker web.

With pleasure we thank V. Serganova for fruitful discussions and for important contributions: the proof of Proposition 14.19, and a significant simplification of the proof of Amplification 7.2. This paper could not have appeared without the long-term joint research with I. M. Gelfand, which molded the mindset of this paper. Special thanks go to A. Panasyuk for providing preprints of [27] and [28], and to B. Okun for patient discussions of results of [11], which lead to a cristallization of crucial ideas of the current paper, and to Y. Flicker for tireless suggestions for improvement.

Revision history. The revisions of this paper are archived as math.SG/9908034 on the math preprint server archive arXiv.org/abs/math. In addition to cosmetic 
changes, revision II of this paper contains a major simplification of arguments in Section 7, adds Remarks 2.5 and 2.6. Section 14 is expanded beyond Corollary 14.24 by adding the discussion of the case of groups of mappings. Revision III reworked the introduction. The numeration of statements did not change.

\section{LiNEAR RELATIONS AND PENCILS}

Definition 2.1. A linear relation $W$ between vector spaces $V_{1}$ and $V_{2}$ is a vector subspace $W \subset V_{1} \oplus V_{2}$. Call $W$ bisurjective if both projections of $W$ to $V_{1}$ and $V_{2}$ are surjective. The left kernel $\operatorname{Ker}_{L} W$ of $W$ is the kernel of the projection $W \rightarrow V_{2}$ considered as a vector subspace of $V_{1}$. The right kernel $\operatorname{Ker}_{R} W \subset V_{2}$ is defined similarly.

A linear relation in $V$ is a linear relation between $V$ and $V$.

Obviously, if $W$ is a linear relation, then $W \supset \operatorname{Ker}_{L} W \oplus \operatorname{Ker}_{R} W$, thus $W$ induces a vector subspace $\widetilde{W}$ of $\left(V_{1} / \operatorname{Ker}_{L} W\right) \oplus\left(V_{2} / \operatorname{Ker}_{R} W\right)$. If $W$ is bisurjective, then $\widetilde{W}$ is a graph of a bijective linear mapping $V_{1} / \operatorname{Ker}_{L} W \stackrel{\sim}{\rightarrow} V_{2} / \operatorname{Ker}_{R} W$. In particular, $\operatorname{dim} V_{1}-\operatorname{dim} V_{2}=\operatorname{dim} \operatorname{Ker}_{L} W-\operatorname{dim} \operatorname{Ker}_{R} W$.

Definition 2.2. Fix once and for all a two-dimensional vector space $\mathcal{S}$ with a basis $\boldsymbol{s}_{1}, \boldsymbol{s}_{2}$. A pencil of linear operators between vector spaces $V$ and $W$ is a linear mapping $V \otimes \mathcal{S} \stackrel{\mathcal{P}}{\rightarrow} W$. This induces two linear mappings $\mathcal{P}_{1,2}: V \rightarrow W$ defined as $\mathcal{P}_{i}(v) \stackrel{\text { def }}{=} \mathcal{P}\left(v \otimes \boldsymbol{s}_{i}\right)$.

Given a pencil $\mathcal{P}$, one obtains a linear 2-parametric family of linear mappings $\lambda_{1} \mathcal{P}_{1}+\lambda_{2} \mathcal{P}_{2}$ between $V$ and $W$. Inversely, any two linear mappings $\mathcal{P}_{1,2}: V \rightarrow W$ correspond to a pencil and to a linear 2-parametric family of linear mappings.

Analyze possible connections between pencils and linear relations in vector spaces. Given a linear relation $W$ in $V$, two projections $\pi_{1,2}$ of $W \subset V \oplus V$ to $V$ define a pencil $\pi$ of operators $W \rightarrow V$. In the other direction, given any pencil $\Pi: W \rightarrow V$, one can construct a linear relation $\widetilde{W} \subset V \oplus V$ as the image of $\Pi_{1} \oplus \Pi_{2}: W \rightarrow$ $V \oplus V$. The pencils $\Pi$ which may obtained from linear relations are those for which $\operatorname{Ker} \Pi_{1} \cap \operatorname{Ker} \Pi_{2}=0$. For such pencils these two constructions are mutually inverse.

Introduce another ${ }^{13}$ connection between bisurjective linear relations in $V$ and pencils of linear operators $V \rightarrow V^{\prime}$ (here $V^{\prime}$ is an arbitrary vector space). Given a bisurjective linear relation $W$ in $V$, one obtains an identification $\alpha: V / \operatorname{Ker}_{R} W \rightarrow$ $V / \operatorname{Ker}_{L} W$. Let $V^{\prime}$ be either $V / \operatorname{Ker}_{R} W$ or $V / \operatorname{Ker}_{L} W$ (or any other vector space identified with both $V / \operatorname{Ker}_{R} W$ and $V / \operatorname{Ker}_{L} W$ in such a way that the identifications commute with $\alpha$ ). To simplify notations, assume $V^{\prime}=V / \operatorname{Ker}_{L} W$.

Denote by $\pi_{1,2}$ the natural projections of $V$ to $V / \operatorname{Ker}_{L} W$ and to $V / \operatorname{Ker}_{R} W$. Then $\mathcal{P}_{1} \stackrel{\text { def }}{=} \pi_{1}, \mathcal{P}_{2}=\alpha \circ \pi_{2}$ give a pair of operators $V \rightarrow V^{\prime}=V / \operatorname{Ker}_{L} W$. In the other direction, given a pencil $\mathcal{P}$ of linear operators $V \rightarrow V^{\prime}$, one can consider a linear

\footnotetext{
${ }^{13}$ In fact, we will not use the previous connection between linear relations and pencils.
} 
relation $W=\mathcal{P}_{2}^{-1} \mathcal{P}_{1}$ (in other words, $\left(v_{1}, v_{2}\right) \in W$ iff $\mathcal{P}_{1} v_{1}=\mathcal{P}_{2} v_{2}$ ). Obviously, this gives two mutually inverse operations between bisurjective relations to pencils.

Obviously, a pencil $\mathcal{P}$ of operators $V \rightarrow V^{\prime}$ can be obtained from a bisurjective linear relation in $V$ iff both linear operators $\mathcal{P}_{1}, \mathcal{P}_{2}$ of the pencil are surjective. Call such pencils bisurjective. In fact, one can obtain a much stronger result:

Definition 2.3. Given a linear relation $W_{1}$ in $V_{1}$ and a linear relation $W_{2}$ in $V_{2}$, call a linear mapping $\varphi: V_{1} \rightarrow V_{2}$ an isomorphism ${ }^{14}$ between $W_{1}$ and $W_{2}$ if $\varphi$ is an isomorphism, and $(\varphi \oplus \varphi)\left(W_{1}\right)=W_{2}$.

Given a pencil $\mathcal{P}$ of operators $V \rightarrow W$ and a pencil $\mathcal{P}^{\prime}$ of operators $V^{\prime} \rightarrow W^{\prime}$ say that linear mappings $\varphi: V \rightarrow V^{\prime}$ and $\psi: W \rightarrow W^{\prime}$ form a morphism between $\mathcal{P}$ and $\mathcal{P}^{\prime}$ if $\mathcal{P}_{1}^{\prime} \varphi=\psi \mathcal{P}_{1}, \mathcal{P}_{2}^{\prime} \varphi=\psi \mathcal{P}_{2}$. Call $(\varphi, \psi)$ an isomorphism if $\varphi$ and $\psi$ form a morphism and $\varphi$ and $\psi$ are isomorphisms of vector spaces.

Proposition 2.4. Consider a category $b s \Re$ of bisurjective linear relations in vector

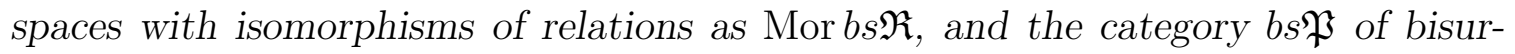
jective pencils of linear mappings with isomorphisms of pencils as Mor bs $\mathfrak{P}$. The mappings bs $\rightarrow b s \mathfrak{P}$ and $b s \mathfrak{P} \rightarrow b s \mathfrak{R}$ defined above give an equivalence of these categories. Moreover, let Vect be the category of vector spaces, consider the functor $b s \Re \rightarrow$ Vect which sends a relation $W$ in $V$ to the vector space $V$, and the functor $b s \mathfrak{P} \rightarrow$ Vect which sends a pencil $\mathcal{P}$ of operators $V \rightarrow W$ to $V$. The equivalence of categories defined above commutes with the mappings to Vect.

In plain words, it is "the same" to consider bisurjective linear relations in $V$ and bisurjective pencils of operators $V \rightarrow V^{\prime}$ up to isomorphisms of $V^{\prime}$.

Remark 2.5. We will use the equivalence of categories in the following way: given a bundle $\mathcal{V}$ over a manifold $M$ with the fibers being vector spaces with bisurjective relations, one can construct a vector bundle $\mathcal{V}^{\prime}$ the whose fibers are vector spaces $V^{\prime}$ of the previous construction. The vector bundle $\mathcal{V}^{\prime}$ is canonically defined.

Remark 2.6. Consider a linear relation $W$ in a vector space $V$, i.e., $W \subset V \oplus V$. Note that $V \oplus V=V \otimes \mathcal{S}$; here $\mathcal{S}$ is the "coordinate" two-dimensional vector space. The group GL $(\mathcal{S})$ acts on $V \otimes \mathcal{S}$, thus acts in the set of linear relations in $V$. This action can be reduced to the action of PGL $(\mathcal{S})$.

On the other hand, GL $(\mathcal{S})$ also acts in the set of pencils $\mathcal{P}$ of operators $V \rightarrow V^{\prime}$, $\left(\mathcal{P}_{1}, \mathcal{P}_{2}\right) \rightarrow\left(a \mathcal{P}_{1}+b \mathcal{P}_{2}, c \mathcal{P}_{1}+d \mathcal{P}_{2}\right)$. Obviously, these two actions are compatible with the mappings given above.

Remark 2.7. One can make the construction of $V^{\prime}$ a little bit more symmetric by using the definition $V^{\prime}=(V \oplus V) / W$.

\footnotetext{
${ }^{14}$ One could consider mappings which are not isomorphisms, but we will not consider such mappings as morphisms.
} 


\section{KRONECKER RELATIONS}

Recall that to any bisurjective linear relation $W$ in $V$ we associated a pencil $\mathcal{P}$ of operators $V \rightarrow V^{\prime}$ (for an appropriate vector space $V^{\prime}$ ). Thus one can consider the corresponding linear family $\lambda_{1} \mathcal{P}_{1}+\lambda_{2} \mathcal{P}_{2}$ of mappings $V \rightarrow V^{\prime}$. In particular, for any $\lambda_{1}$ and $\lambda_{2}$ one obtains a vector subspace $\operatorname{Ker}\left(\lambda_{1} \mathcal{P}_{1}+\lambda_{2} \mathcal{P}_{2}\right) \subset V$. Denote this subspace by $\operatorname{Ker}_{\lambda_{1}: \lambda_{2}} W$. Obviously, for $\left(\lambda_{1}, \lambda_{2}\right) \neq(0,0)$ this subspace depends only on the ratio $\left(\lambda_{1}: \lambda_{2}\right) \in \mathbb{P}^{1}$, and $\operatorname{Ker}_{L}=\operatorname{Ker}_{\mathcal{P}_{1}}=\operatorname{Ker}_{1: 0}, \operatorname{Ker}_{R}=\operatorname{Ker}_{0: 1}$. Moreover, if the vector spaces we consider are defined over a field $\mathbb{K}$, in fact one can consider $\lambda_{1}$ and $\lambda_{2}$ to be in any extension $\mathbb{E}$ of $\mathbb{K}$, then $\operatorname{Ker}_{\lambda_{1}: \lambda_{2}} W \subset V \otimes_{\mathbb{K}} \mathbb{E}$.

In particular, if $\overline{\mathbb{K}}$ is the algebraic closure of $\mathbb{K}$, then for any $\lambda=\left(\lambda_{1}: \lambda_{2}\right) \in \overline{\mathbb{K}} \mathbb{P}^{1}$ one can consider a correctly defined number $\operatorname{dim} \operatorname{Ker}_{\lambda} W$.

Definition 3.1. A bisurjective linear relation $W$ in $V$ is Kronecker if $\operatorname{dim}_{K^{\prime}} W$ does not depend on $\lambda \in \mathbb{K} \mathbb{P}^{1}$. Call this common dimension the rank of the relation.

Example 3.2. Assume that $W$ is a graph of a linear mapping $V \rightarrow V$. Then $W$ is Kronecker iff $\operatorname{dim} V=0$.

Example 3.3. Let $V=\mathbb{K}^{n}$. Define $W \subset V \oplus V$ by $\left(v, v^{\prime}\right) \in W$ iff $v_{k}=v_{k+1}^{\prime}$, $k=1, \ldots, n-1$. This is a Kronecker linear relation of rank 1 .

Definition 3.4. A Kronecker block is a linear relation isomorphic to the relation of Example 3.3.

Definition 3.5. Given a linear relation $W$ in $V$ and a linear relation $W^{\prime}$ in $V^{\prime}$, $W \oplus W^{\prime}$ can be considered as a linear relation in $V \oplus V^{\prime}$. Call this linear relation a direct sum of $W$ and $W^{\prime}$.

Definition 3.6. For $\lambda \in \mathbb{K}$ say that a relation $W$ in $V$ is a Jordan block with eigenvalue $\lambda$ if $W$ is a graph of a mapping $V \rightarrow V$ which is a Jordan block with eigenvalue $\lambda$. Say that $W$ is a Jordan block with eigenvalue $\infty$ if $W^{-1}$ is a Jordan block with eigenvalue 0 ; here $W^{-1}$ is the image of $W$ under transposition of summands in $V \oplus V$.

Theorem 3.7. Suppose that $\mathbb{K}$ is algebraically closed and $\operatorname{dim} V<\infty$.

1. any Kronecker linear relation in $V$ of rank 1 is a Kronecker block.

2. Any Kronecker linear relation in $V$ is isomorphic to a direct sum of Kronecker blocks.

3. Any bisurjective linear relation is isomorphic to a direct sum of Kronecker and Jordan blocks.

The collection of dimensions (and - for Jordan blocks - eigenvalues) of these blocks is uniquely determined by $V$.

Proof. See classification of pencils of finite-dimensional linear operators, say in [9] or [36]. 
Given a Kronecker relation $W$ in $V$ of rank $r$, one obtains a natural mapping Ker $: \mathbb{P}^{1} \rightarrow \operatorname{Gr}_{r} V$. Call this parameterized curve in $\operatorname{Gr}_{r} V$ the spectral curve of $W$.

Remark 3.8. Given a bisurjective relation $W$ in a finite-dimensional vector space $V$, $\operatorname{dim} \operatorname{Ker}_{\lambda} W$ is constant on a Zariski open subset of $\overline{\mathbb{K}} \mathbb{P}^{1}$, i.e., outside of a finite subset $\Lambda \subset \overline{\mathbb{K}} \mathbb{P}^{1}$. In particular, there is a natural mapping Ker $: \mathbb{P}^{1} \backslash \Lambda \rightarrow \mathrm{Gr}_{r} V$. Since $\mathrm{Gr}_{r} V$ is complete, one can extend this mapping to a mapping $\mathbb{P}^{1} \rightarrow \mathrm{Gr}_{r} V$, which one can call a spectral curve as well.

Proposition 3.9. Consider two matrices $A_{1}, A_{2} \in \operatorname{Mat}(m, n)$. They define a pencil of mappings $\overline{\mathbb{K}}^{m} \rightarrow \overline{\mathbb{K}}^{n}$. Suppose that $m \geq n$ and $\operatorname{rk}\left(\lambda_{1} A_{1}+\lambda_{2} A_{2}\right)=n$ for any $\left(\lambda_{1}, \lambda_{2}\right) \in \mathbb{K}^{2} \backslash(0,0)$. Then the same is true for any pair $\left(A_{1}^{\prime}, A_{2}^{\prime}\right)$ which is close to $\left(A_{1}, A_{2}\right)$.

Proof. Consider the projectivization $\mathbb{P}$ Mat $(m, n)$ of the vector space of matrices. Let $Z \subset \mathbb{P}$ Mat $(m, n)$ be the projection of the set of matrices of rank $<n$ to $\mathbb{P} \operatorname{Mat}(m, n)$, denote projections of $A_{1}, A_{2}$ on $\mathbb{P} \operatorname{Mat}(m, n)$ by $\alpha_{1}, \alpha_{2}$. Then $Z$ is a closed subset, and the line through $\alpha_{1}, \alpha_{2}$ does not intersect $Z$. Due to compactness of $\mathbb{P} \operatorname{Mat}(m, n)$, nearby lines do not intersect $Z$ as well.

Remark 3.10. Due to the correspondence of Proposition 2.4, one can restate Proposition 3.9 as the fact that Kronecker relations in a vector space $V$ form an open subset of all relations in $V$. Here we identify the set of relations with the union of Grassmannians of subspaces of different dimensions in $V \oplus V$.

\section{KRONECKER WEBS}

Definition 4.1. A preweb on a manifold $B$ is a bisurjective linear relation in $\mathcal{T}^{*} B$, in other words, it is a vector bundle $\mathcal{W}$ on $B$ with an inclusion $\mathcal{W} \hookrightarrow \mathcal{T}^{*} B \oplus \mathcal{T}^{*} B$ which makes a bisurjective linear relation in each fiber of $\mathcal{T}^{*} B$.

Given a preweb $\mathcal{W}$ and $\lambda \in \mathbb{K P}^{1}$, one can consider $\operatorname{Ker}_{\lambda} \mathcal{W}$, which is a collection of vector subspaces $\operatorname{Ker}_{\lambda} \mathcal{W}_{b} \subset \mathcal{T}_{b}^{*} B, b \in B$. Recall that $\mathcal{N} \mathcal{F}, \mathcal{T} \mathcal{F}$, and $\mathcal{T}^{*} \mathcal{F}$ for a foliation $\mathcal{F}$ were defined in Section 0 .

Definition 4.2. Consider a vector subbundle $E$ of $\mathcal{T}^{*} B$. Call $E$ integrable if there is a foliation $\mathcal{F}$ on $B$ such that $E=\mathcal{N F}$.

Definition 4.3. A preweb $\mathcal{W}$ is a web if for any given $\lambda \in \mathbb{K} \mathbb{P}^{1}$ the number $\operatorname{dim} \operatorname{Ker}_{\lambda} \mathcal{W}_{b}$ does not depend on $b \in B$, and this collection of subspaces is integrable. In other words, there is a foliation $\mathcal{F}_{\lambda}$ on $B$ such that $\operatorname{Ker}_{\lambda} \mathcal{W}_{b}$ coincides with the normal spaces to $\mathcal{F}_{\lambda}$ at $b \in B$. Call this foliation the $\lambda$-integrating foliation (or just the integrating foliation, if $\lambda$ is clear from the context) of $\mathcal{W}$.

A preweb $\mathcal{W}$ is Kronecker of rank $r$ if for any $b \in B$ the linear relation $\mathcal{W}_{b}$ in $\mathcal{T}_{b}^{*} B$ is Kronecker of $\operatorname{rank}^{15} r$.

\footnotetext{
${ }^{15}$ Since rank of a Kronecker relation $W$ in $V$ equals $\operatorname{dim} W-\operatorname{dim} V$, rank of $\mathcal{W}_{m} \operatorname{does}$ not depend on $m$.
} 
Remark 4.4. Note that Theorem 7.1 implies that the notion of Kronecker webs is a generalization of the notion of Veronese webs of higher codimension introduced in [28]. Since the definition of [28] cannot not stated in a coordinate-independent form, Definition 4.3 is much more convenient to work with.

Due to Proposition 2.4, to describe a preweb $\mathcal{W}$ on $B$ is "the same" as to define a vector bundle $\Phi(\mathcal{W})$ on $B$ and a pencil $\mathcal{P}$ of bisurjective mappings of vector bundles $\mathcal{T}^{*} B \rightarrow \Phi(\mathcal{W})$. If the preweb $\mathcal{W}$ is clear from the context, we may denote $\Phi(\mathcal{W})$ as $\Phi$ as well. ${ }^{16}$ Recall that given $\mathcal{W}, \Phi(\mathcal{W})=\mathcal{T}^{*} B / \operatorname{Ker}_{\lambda} \mathcal{W}$ (here $\lambda$ is any number fixed in advance), given $\Phi$ and $\mathcal{P}$, the vector bundle $\mathcal{W} \subset \mathcal{T}^{*} B \oplus \mathcal{T}^{*} B$ can be described by the condition $\mathcal{P}_{1, b} v_{1}=\mathcal{P}_{2, b} v_{2},\left(v_{1}, v_{2}\right) \in \mathcal{T}_{b}^{*} B$.

By definition, this preweb is Kronecker if $\operatorname{Ker}\left(\lambda_{1} \mathcal{P}_{1, b}+\lambda_{2} \mathcal{P}_{2, b}\right), b \in B$, is an family of subspaces of $\mathcal{T}^{*} B$ (or $\mathcal{T}^{*} B \otimes \mathbb{C}$ ) of the same dimension for any $b \in B$ and $\left(\lambda_{1}: \lambda_{2}\right) \in$ $\mathbb{C P}^{1}$. If $\mathcal{W}$ is a Kronecker preweb, $\mathcal{W}$ is a Kronecker web if $\operatorname{Ker}\left(\lambda_{1} \mathcal{P}_{1, b}+\lambda_{2} \mathcal{P}_{2, b}\right)$ is an integrable family of subspaces of $\mathcal{T}^{*} B$ of the same dimension for any $b \in B$ and $\left(\lambda_{1}: \lambda_{2}\right) \in \mathbb{K} \mathbb{P}^{1}$. Due to Remark 3.10, the condition on a (pre)web to be Kronecker is a condition of being in general position.

For a given $b \in B$ one can define $\Lambda_{b} \subset \mathbb{C P}^{1}$ as in Remark 3.8. Obviously, if $\lambda_{0} \notin \Lambda_{b_{0}}$, then there are neighborhoods $U \ni b_{0}$ and $\boldsymbol{U} \ni \lambda_{0}$ such that the vector subspaces $\operatorname{Ker}_{\lambda} \mathcal{W}_{b}$ depend smoothly on $b \in U$ and $\lambda \in \boldsymbol{U}$. For any given $\left(\lambda_{1}: \lambda_{2}\right) \in \boldsymbol{U} \subset \mathbb{C P}^{1}$ the bundle $\mathcal{T}^{*} B /\left.\operatorname{Ker}_{\left(\lambda_{1}: \lambda_{2}\right)} \mathcal{W}\right|_{U}$ is canonically isomorphic to $\left.\Phi\right|_{U}$. On the other hand, if $\lambda \in \mathbb{K} \mathbb{P}^{1}$, and $\mathcal{W}$ is a web, then $\mathcal{T}^{*} B / \operatorname{Ker}_{\left(\lambda_{1}: \lambda_{2}\right)} \mathcal{W}=\mathcal{T}^{*} \mathcal{F}_{\left(\lambda_{1}: \lambda_{2}\right)}$.

We see that given $\left(\lambda_{1}, \lambda_{2}\right) \in \mathbb{K}^{2} \backslash(0,0)$ such that $\lambda=\left(\lambda_{1}: \lambda_{2}\right) \in \boldsymbol{U}$ the vector bundle $\left.\Phi\right|_{U}$ is canonically identified with $\left.\mathcal{T}^{*} \mathcal{F}_{\lambda}\right|_{U}$. Since $\mathcal{T}^{*} \mathcal{F}$ has a natural Poisson structure (see Section 0), we see that

Proposition 4.5. The total space $\left.\Phi\right|_{U}$ carries a natural Poisson structure for any $\left(\lambda_{1}, \lambda_{2}\right) \in \mathbb{K}^{2} \backslash(0,0)$ such that $\lambda=\left(\lambda_{1}: \lambda_{2}\right) \in \boldsymbol{U}$. This Poisson structure depends smoothly on $\left(\lambda_{1}, \lambda_{2}\right)$.

Call this Poisson structure $\eta_{\lambda_{1}, \lambda_{2}}$. Let $\widetilde{U}=\left\{\left(\lambda_{1}, \lambda_{2}\right) \mid \lambda_{1}: \lambda_{2} \in U\right\}$.

Proposition 4.6. The Poisson structure $\eta_{\lambda_{1}, \lambda_{2}}$ is homogeneous in $\left(\lambda_{1}, \lambda_{2}\right) \in \widetilde{U}$ of degree 1.

Proof. Indeed, multiplication of $\left(\lambda_{1}, \lambda_{2}\right)$ by a constant changes the identification of $\left.\Phi\right|_{U}$ with $\left.\mathcal{T}^{*} \mathcal{F}_{\lambda}\right|_{U}$ by the same constant. For a diffeomorphism $\alpha: M_{1} \rightarrow M_{2}$ and a Poisson structure $\eta$ on $M_{1}$ denote by $\alpha_{*} \eta$ the Poisson structure $\eta$ transferred to $M_{2}$ via $\alpha, \alpha_{*} \eta(f, g) \stackrel{\text { def }}{=} \eta(f \circ \alpha, g \circ \alpha) \circ \alpha^{-1}$. Apply this definition in the case when $\alpha=\mu_{c}$ is multiplication by $c$ in $\mathcal{T}^{*} N$.

\footnotetext{
${ }^{16}$ The choice of notation is related to the fact that in applications the coordinates on fibers of $\Phi$ are angle-coordinates of an integrable system. (The action variables are coordinates on the global space of $\Phi$ coming from coordinates on $M$.)
} 
Now the only thing to prove is that $\left(\mu_{c}\right)_{*} \eta=c \eta$ if $\eta$ is the canonical Poisson structure on $\mathcal{T}^{*} N$, and $N$ is an arbitrary manifold. One can check it momentarily in local coordinates on $N$ (as in Example 0.6).

Corollary 4.7. If $\mathbb{K}=\mathbb{C}$, and the web $\mathcal{W}$ is Kronecker, then the Poisson structure $\eta_{\lambda_{1}, \lambda_{2}}$ on the total space of $\Phi$ depends linearly on $\lambda_{1}, \lambda_{2}$.

Proof. Since for a Kronecker web $\Lambda_{b}=\varnothing$, one can take $\boldsymbol{U}=\mathbb{C P}^{1}$, and $U=B$, thus the Poisson structure $\eta_{\lambda_{1}, \lambda_{2}}$ is defined on the whole total space of $\Phi$ for any $\left(\lambda_{1}, \lambda_{2}\right) \neq(0,0)$. Fix $x \in \Phi$. Since a Poisson structure is a bivector field, one can associate to $\left(\lambda_{1}, \lambda_{2}\right) \neq(0,0)$ an element $\left.\eta_{\lambda_{1}, \lambda_{2}}\right|_{x}$ of $\Lambda^{2} \mathcal{T}_{x} \Phi$. We know that this element depends analytically on $\left(\lambda_{1}, \lambda_{2}\right) \neq(0,0)$ and is homogeneous of degree 1 in $\left(\lambda_{1}, \lambda_{2}\right)$. However, any mapping of $\mathbb{C}^{2} \backslash(0,0)$ to a vector space which is of homogeneity degree 1 is linear, which finishes the proof.

Theorem 4.8. If a web $\mathcal{W}$ is Kronecker, then the Poisson structure $\eta_{\lambda_{1}, \lambda_{2}}$ on the total space of $\Phi$ depends linearly on $\lambda_{1}, \lambda_{2}$.

Proof. The only case which remains to be proven is $\mathbb{K}=\mathbb{R}$. If a Kronecker web $\mathcal{W}$ is real-analytic, then one can consider the complex-analytic continuation, and one returns to the case $\mathbb{K}=\mathbb{C}$. Thus in real-analytic case Corollary 4.7 implies the theorem.

Consider now $C^{\infty}$-case. First, note that the theorem follows from some "abstract nonsense" remarks. Recall that one of the contributions of algebraic geometry to differential geometry is the understanding of the importance of considering "formal" objects as tools for investigation of "geometric" objects. In particular, given a $C^{\infty}$ manifold, one can consider "an $\infty$-jet of the complex-analytic neighborhood" (or a "formal neighborhood") of this manifold. This formal neighborhood is canonically defined, and carries many properties of complex-analytic manifolds.

Readers familiar with the language we used above may immediately recognize that all the objects needed for the proof of Corollary 4.7 (foliations, tensors, cotangent bundles, Poisson structures) make sense in settings of "formal geometry", thus one can finish the proof in $C^{\infty}$-case in the same way we did it in real-analytic case (when we had a no-nonsense complex neighborhood instead of a formal one). For other readers we provide a stripped-down version of the proof below. This version will not use "hard" notions of formal geometry (such as jets of manifolds etc.), but will use only notions of (finite-order) jets of sections of "real" bundles on "real" manifolds. ${ }^{17}$ This simplified version of the proof goes until the end of this section.

Consider a vector bundle $E$ over $B$, fix a point $b \in B$. To describe a vector subbundle $F$ of $E$ of rank $d$ is the same as to describe a section of the bundle $\operatorname{Gr}_{d}(E)$ (the fibers of this bundle are Grassmannians of fibers of $F$ ). Recall that a $k$-jet near $b$ of a section $f$ of a bundle is the collection of Taylor coefficients of $f$ of the order $k$ or

\footnotetext{
${ }^{17}$ As opposed to "formal" manifolds.
} 
less. ${ }^{18}$ If a geometric object can be described locally by a section of some bundle, one can define a $k$-jet of this object near $b$ as being a $k$-jet of a section of this bundle ${ }^{19}$. Thus one can define a $k$-jet of a vector subbundle $F$ of $E$ near $b$.

Remark 4.9. In what follows we will also need to consider $E / F$; here $F$ is a $(k$-jet of) a subbundle of $E$. To avoid introducing some new "abstract nonsense", equip $E$ with a norm, and identify $E / F$ with the orthogonal complement to $F$. Obviously, given a $k$-jet of $F$, the $k$-jet of $E / F$ is well-defined as a $k$-jet of a vector (sub)bundle.

In particular, one can define a $k$-jet $\mathcal{W}$ of a preweb. Consider two prewebs $\mathcal{W}$ and $\mathcal{W}^{\prime}$ which have the same $k$-jet near $b$. Then the subbundles $\operatorname{Ker}_{\lambda} \mathcal{W} \subset \mathcal{T}^{*} B$ and $\operatorname{Ker}_{\lambda} \mathcal{W}^{\prime} \subset \mathcal{T}^{*} B$ have the same $k$-jet near $b$. Thus there is a canonically defined $k$-jet of an isomorphism $\iota$ between vector bundles $\mathcal{T}^{*} B / \operatorname{Ker}_{\lambda} \mathcal{W}$ and $\mathcal{T}^{*} B / \operatorname{Ker}_{\lambda} \mathcal{W}^{\prime}$. This leads to a $k$-jet ${ }^{20}$ of an isomorphism between the total spaces of these bundles. On the other hand, given a $k$-jet of an isomorphism $\iota$ between two manifolds $F$ and $F^{\prime}$, and a tensor field $\tau$ on $F$, one can define a $k-1$-jet of the tensor field $\iota_{*} \mathcal{T}$ on $F^{\prime}$.

Suppose that both prewebs $\mathcal{W}$ and $\mathcal{W}^{\prime}$ are in fact webs. As we know, total spaces of both bundles $\mathcal{T}^{*} B / \operatorname{Ker}_{\lambda} \mathcal{W}$ and $\mathcal{T}^{*} B / \operatorname{Ker}_{\lambda} \mathcal{W}^{\prime}$ carry natural Poisson structures, and one can consider Poisson structures as bivector (thus tensor) fields $\eta$ and $\eta^{\prime}$. Thus given a $k$-jet $\iota$ of an isomorphism between these manifolds, one can consider $\iota_{*} \eta$, which is a $k-1$-jet of a Poisson structure on $\mathcal{T}^{*} B / \operatorname{Ker}_{\lambda} \mathcal{W}^{\prime}$. Obviously, $\iota_{*} \eta$ coincides with the $k-1$-jet of $\eta^{\prime}$. This immediately implies

Lemma 4.10. Consider two Kronecker webs $\mathcal{W}$ and $\mathcal{W}^{\prime}$ on $B$ which have the same $k$-jet near $b \in B$. Then there is a canonically defined $k$-jet of an isomorphism $\iota$ between $\Phi(\mathcal{W})$ and $\Phi\left(\mathcal{W}^{\prime}\right)$, and this isomorphism identifies $k-1$-jets of Poisson structures $\eta_{\lambda_{1}, \lambda_{2}}$ (on $\Phi$ ) and $\eta_{\lambda_{1}, \lambda_{2}}^{\prime}$ (on $\Phi^{\prime}$ ) near fibers of these bundles over $b$.

Thus

Lemma 4.11. In the conditions of Lemma 4.10 suppose that $k=1$, and the web $\mathcal{W}^{\prime}$ is in fact real-analytic. Then the tensor field $\eta_{\lambda_{1}, \lambda_{2}}$ on the fiber of $\Phi$ over $b$ depends linearly on $\left(\lambda_{1}, \lambda_{2}\right)$.

In particular, the theorem follows from the case $k=1$ of

Conjecture 4.12. Fix $k \geq 0$. Given a Kronecker web $\mathcal{W}$ on an open subset $U \subset \mathbb{R}^{n}$, $0 \in U$, one can find a real-analytic Kronecker web $\mathcal{W}^{\prime}$ on $U^{\prime} \subset U, 0 \in U^{\prime}$, such that $k$-jets of $\mathcal{W}$ and $\mathcal{W}^{\prime}$ at 0 coincide.

\footnotetext{
${ }^{18}$ In a coordinate-less form, $k$-jets are equivalence classes of sections; here the equivalence is having the same Taylor coefficients of order $k$ or less in any coordinate system.

${ }^{19}$ However, it may happens that there are different descriptions of the same object which lead to mutually shifted enumerations of jets. For example, locally one can describe a closed 1-form $\alpha$ by a function $f$ such that $d f=\alpha$, or by components of $\alpha$ considered as a tensor field. The $k$-jet in the first description is a $k-1$-jet in the second description.

${ }^{20} \mathrm{It}$ is possible to define what is a $k$-jet of something near a submanifold, and the $k$-jet in question is defined near two fibers over $b$ of these two vector bundles.
} 
In fact, in the case $k=1$ we propose the following amplification:

Conjecture 4.13. Given a Kronecker web $\mathcal{W}$ on an open subset $U \subset \mathbb{R}^{n}, 0 \in U$, one can find $U^{\prime} \subset U, 0 \in U^{\prime}$, and a diffeomorphism $U^{\prime} \stackrel{f}{\rightarrow} V \subset \mathbb{R}^{n}, f(0)=0$, such that 1-jet of $f_{*} \mathcal{W}$ (the transfer of $\mathcal{W}$ via $f$ ) coincides with 1-jet of an appropriate translation-invariant ${ }^{21}$ Kronecker web $\mathcal{W}^{\prime}$.

Not only this conjecture implies the case $k=1$ of Conjecture 4.12 , but it would also directly imply the theorem we are proving. However, since these conjectures are not settled yet $^{22}$, one needs an alternative way to prove the theorem.

We are going to do this without the extension-properties for webs which are mentioned above by introducing integrability conditions on $k$-jets of prewebs. Since jets are just collections of numbers, thus are not sensible to a change of the base field, we will be able to consider a $k$-jet of a $C^{\infty}$-web as a $k$-jet of a complex-analytic web, thus we will be able to extend $\eta_{\lambda_{1}, \lambda_{2}}$ to $\left(\lambda_{1}, \lambda_{2}\right) \in \mathbb{C}^{2} \backslash(0,0)$, then apply the arguments of Corollary 4.7.

Definition 4.14. A $k$-jet $K$ of a vector subbundle of $\mathcal{T}^{*} B$ is $k$-integrable near $b_{0} \in B$ if there is a foliation $\mathcal{F}$ such that the $k$-jet of the normal bundle to $\mathcal{F}$ coincides with $K$.

Definition 4.15. A $k$-jet $\mathcal{W}$ of a preweb is a $k$-jet-web if for any $\lambda \in \mathbb{K} \mathbb{P}^{1}$ the $k$-jet $\operatorname{Ker}_{\lambda} \mathcal{W}$ of a vector subbundle of $\mathcal{T}^{*} B$ is $k$-integrable. A $k$-jet $\mathcal{W}$ of a web near $b$ is Kronecker if the relation $\mathcal{W}_{b}$ in $\mathcal{T}_{b}^{*} B$ is Kronecker.

Due to Remark 3.10, if a $k$-jet near $b$ of preweb $\mathcal{W}$ on $B$ is Kronecker, then the preweb $\mathcal{W}$ is Kronecker in a neighborhood of $b$. Thus the definition of a Kronecker $k$-jet-web above is compatible with taking a $k$-jet of a Kronecker web.

Consider a $k$-jet-web $\mathcal{W}$. It is a $k$-jet of a preweb, denote this preweb by $\mathcal{W}^{\prime}$. Given $\left(\lambda_{1}, \lambda_{2}\right) \in \mathbb{K}^{2} \backslash(0,0)$, the $k$-jet of $\mathcal{T}^{*} B / \operatorname{Ker}_{\lambda} \mathcal{W}^{\prime}, \lambda=\lambda_{1}: \lambda_{2}$, is naturally identified with the $k$-jet of the cotangent bundle of the corresponding foliation $\mathcal{F}_{\lambda}$, in other words, one can construct a $k$-jet of an identification $\alpha$ of $\mathcal{T}^{*} B / \operatorname{Ker}_{\lambda} \mathcal{W}^{\prime}$ with $\mathcal{T}^{*} \mathcal{F}_{\lambda}$. Recall that a $k$-jet of a diffeomorphism acts on $k$-1-jets of tensor fields. $\mathcal{T}^{*} \mathcal{F}_{\lambda}$ carries a natural Poisson structure, thus it carries the tensor field which describes the bracket of the Poisson structure. Using the identification $\alpha$ mentioned above, one obtains a $k-1$-jet of a tensor field $\eta_{\lambda_{1}, \lambda_{2}}$ on $\mathcal{T}^{*} B / \operatorname{Ker}_{\lambda} \mathcal{W}^{\prime}$. Obviously, one can consider this $k-1$-jet as living on $\mathcal{T}^{*} B / \operatorname{Ker}_{\lambda} \mathcal{W}$. It does not depend on the choice of $\mathcal{W}^{\prime}$.

\footnotetext{
${ }^{21} \mathrm{~A}$ Kronecker web $\mathcal{W}$ on a vector space $V$ is translation-invariant if the vector subspace $\mathcal{W}_{v} \subset$ $\mathcal{T}_{v}^{*} V \oplus \mathcal{T}_{v}^{*} V=V^{*} \oplus V^{*}$ does not depend on $v \in V$.

${ }^{22}$ As Proposition 4.17 implies, the condition on a preweb of being a web can be written as a system of differential equations in partial derivatives. These systems are non-linear, and in all but a handful of cases they are enormously overdetermined.

This makes the conjectures above so hard to tackle.
} 
If $\mathbb{K}=\mathbb{C}$, and $k=1$, then one obtains a 0 -jet ${ }^{23}$ of a tensor field $\eta_{\lambda_{1}, \lambda_{2}}$ for any $\left(\lambda_{1}, \lambda_{2}\right) \in \mathbb{C}^{2} \backslash(0,0)$, and the same arguments as in the proof of Corollary 4.7 show that

Proposition 4.16. Let $\mathbb{K}=\mathbb{C}$, consider a Kronecker 1 -jet-web $\mathcal{W}$ near $b_{0} \in B$. The family of tensors fields $\eta_{\lambda_{1}, \lambda_{2}}$ on the total space of $\Phi$ is well defined on the fiber over $b_{0}$ of the projection $\Phi \rightarrow B$. This family depends linearly on $\lambda_{1}, \lambda_{2}$.

Proof. The only thing to prove is that $\eta_{\lambda_{1}, \lambda_{2}}$ depends smoothly on $\lambda_{1}, \lambda_{2}$. The definition of $\eta_{\lambda_{1}, \lambda_{2}}$ depended on the foliation $\mathcal{F}_{\lambda}$, and a priori we have no conditions on how $\mathcal{F}_{\lambda}$ depends on $\lambda$. It is enough to show that the $k$-jet of $\mathcal{F}_{\lambda}$ depends smoothly on $\lambda$. Any foliation $\mathcal{F}$ on a manifold $M$ can be locally described by equations $Y=F(X, T)$; here $\left(x_{1}, \ldots, x_{k}, y_{1}, \ldots, y_{r}\right)$ is a local coordinate system, $X=\left(x_{1}, \ldots, x_{k}\right), Y=\left(y_{1}, \ldots, y_{r}\right), T=\left(t_{1}, \ldots, t_{r}\right)$, and $F(0, T) \equiv T$. If we know the subbundle $\mathcal{N} \mathcal{F} \subset \mathcal{T}^{*} M$, then we know that

$$
\frac{\partial F}{\partial X}=A(x, F)
$$

here $A$ is a matrix function of $k+r$ variables which can be deduced from equations of $\mathcal{N} \mathcal{F}$ in $\mathcal{T}^{*} M$. Given $A$, one can find the unique solution of a part of (4.1) with initial conditions $F(0, T) \equiv T$ : Require that $\partial F / \partial x_{l}$ is given by $(4.1)$ if $x_{l+1}=\cdots=x_{k}=0$. Moreover, given a $k$-jet of $A$, this uniquely determines $k+1$-jet of $F$.

This implies that if there is a family of foliations $\mathcal{F}^{(\mu)}$ and the subbundle $\mathcal{N} \mathcal{F}^{(\mu)} \subset$ $\mathcal{T}^{*} M$ depends smoothly on $\mu$, then $\mathcal{F}^{(\mu)}$ depends smoothly on $\mu$. In turn, this implies that $\eta_{\lambda_{1}, \lambda_{2}}$ depends smoothly on $\lambda_{1}, \lambda_{2}$.

To finish the proof of the theorem, it is enough to show that given a Kronecker 1 -jet-web over $\mathbb{R}$, one can consider it as a Kronecker 1 -jet-web over $\mathbb{C}$. Recall that a jet of a preweb is just a collection of Taylor coefficients of a section of a bundle, thus a collection of numbers. Any collection of real numbers can be considered as a collection of complex numbers, thus a 1-jet of a preweb over $\mathbb{R}$ can be considered as 1 -jet of a preweb over $\mathbb{C}$. Since the condition of being Kronecker does not change when we change field of scalars, the only thing we need to prove is the integrability condition for complex $\lambda_{1}: \lambda_{2}$.

Recall Frobenius integrability condition:

Proposition 4.17. Consider a vector subbundle $E$ of $\mathcal{T}^{*} B$. Suppose that for any small open subset $U \subset B$ and any section $\alpha$ of $E$ over $U$ its de Rham differential $d \alpha$ (which is a 2-form on $B$ ) can be written as $d \alpha=\sum \alpha_{i} \wedge \beta_{i}$ with $\alpha_{i}$ being sections of $E$ and $\beta_{i}$ being arbitrary differential forms on $B$. Then $E$ is integrable.

By constructing jet-solutions of ordinary differential equations, one can easily prove the following jet-analogue of Proposition 4.17:

\footnotetext{
${ }^{23}$ Which is a tensor field on the total space of $\Phi$ defined on the fiber over $b$ only.
} 
Proposition 4.18. Consider a $k$-jet of a vector subbundle $E$ of $\mathcal{T}^{*} B$ near $b \in B$. Suppose that for any open subset $U \subset B$ and for any $k$-jet $\alpha$ of a section of $E$ near $b \in B$ its de Rham differential $d \alpha$ (which is a $k-1$-jet of a 2-form on $B$ ) can be written as $\sum \alpha_{i} \wedge \beta_{i}$ with $\alpha_{i}$ being $k-1$-jets of sections of $E$ and $\beta_{i}$ being arbitrary differential forms on $B$. Then $E$ is $k$-integrable.

In fact one can do more. Given a section $\alpha$ of $E$, consider the image of $d \alpha$ under projection $\Omega^{2} B=\Lambda^{2} \mathcal{T}^{*} B \rightarrow \Lambda^{2}\left(\mathcal{T}^{*} B / E\right)$. This defines a mapping $\delta$ from sections of $E$ to sections of $\Lambda^{2}\left(\mathcal{T}^{*} B / E\right)$. A priori it is a differential operator of order 1 , but in fact it has order 0 , thus is a linear mapping between bundles. Indeed, if $\left.\alpha\right|_{b}=0$, then one can easily check that $\left.\delta \alpha\right|_{b}=0$.

Thus $\delta$ defines a section of the vector bundle $E^{*} \otimes \Lambda^{2}\left(\mathcal{T}^{*} B / E\right)$, call this section the torsion of $E$. Given a $k$-jet of a vector subbundle $E \subset \mathcal{T}^{*} B, \delta$ is defined as $k-1$-jet of a section of $E^{*} \otimes \Lambda^{2}\left(\mathcal{T}^{*} B / E\right)$. If a vector subbundle $E_{t}$ depends smoothly on a parameter $t \in T$, then $\delta_{t}$ depends smoothly on $t$ (i.e., it is a smooth section of the vector bundle $E_{t}^{*} \otimes \Lambda^{2}\left(\mathcal{T}^{*} B / E_{t}\right)$ over $\left.B \times T\right)$.

Suppose $k \geq 1$. Apply the construction of $\delta$ above to the vector subbundle $\operatorname{Ker}_{\lambda} \mathcal{W} \subset \mathcal{T}^{*} B$ of a Kronecker preweb $\mathcal{W}$ in the case $\mathbb{K}=\mathbb{C}$. Here $\lambda \in \mathbb{C P}^{1}$, thus $\delta$ is a section of $\left(\operatorname{Ker}_{\lambda} \mathcal{W}\right)^{*} \otimes \Lambda^{2}\left(\mathcal{T}^{*} B / \operatorname{Ker}_{\lambda} \mathcal{W}\right)$ over $B \times \mathbb{C P}^{1}$. Restrict this section to $\{b\} \times \mathbb{C P}^{1}, b \in B$. We obtain a vector bundle over $\mathbb{C P}^{1}$, and a regular section $\delta^{(b)}$ of this vector bundle. There may be only two different cases: either $\delta^{(b)} \equiv 0$, or $\delta^{(b)}$ vanishes at a finite number of points of $\mathbb{C P}^{1}$.

Proposition 4.19. Consider a Kronecker 1 -jet-web $\mathcal{W}$ near $0 \in \mathbb{R}^{n}$ (and $\mathbb{K}=\mathbb{R}$ ). The complexification of this 1-jet-web is a 1-jet $\mathcal{W}_{\mathbb{C}}$ of a Kronecker preweb with $\mathbb{K}=\mathbb{C}$. Then $\mathcal{W}_{\mathbb{C}}$ is a Kronecker 1 -jet-web.

Proof. Arbitrarily extend $\mathcal{W}$ to a preweb $\mathcal{W}^{\prime}$ in neighborhood of 0 in $\mathbb{R}^{n}$, and arbitrarily extend $\mathcal{W}_{\mathbb{C}}$ to a preweb $\mathcal{W}_{\mathbb{C}}^{\prime}$ in a neighborhood of 0 in $\mathbb{C}^{n}$. Consider the torsion $\delta_{\mathbb{C}}$ of the subbundles $\operatorname{Ker}_{\lambda} \mathcal{W}_{\mathbb{C}}^{\prime}$ restricted to $\{0\} \times \mathbb{C} \mathbb{P}^{1}$, and the torsion $\delta$ of the subbundles $\operatorname{Ker}_{\lambda} \mathcal{W}^{\prime}$ restricted to $\{0\} \in \mathbb{R} \mathbb{P}^{1}$. Obviously, $\delta$ is a restriction of $\delta_{\mathbb{C}}$ from $\mathbb{C P}^{1}$ to $\mathbb{R P}^{1}$. On the other hand, $\delta$ vanishes, since 1 -jet $\mathcal{W}$ of $\mathcal{W}^{\prime}$ is a 1 -jet-web. Thus $\delta_{\mathbb{C}}$ vanishes on $\mathbb{R P}^{1}$, thus at infinitely many points of $\mathbb{C P}^{1}$, thus $\delta_{\mathbb{C}}=0$. This implies that the conditions of Proposition 4.18 are satisfied, which finishes the proof.

This finishes the proof of Theorem 4.8.

In the proof above we ignored the question of possibility of extension of jets of webs completely. Let us state

Conjecture 4.20. Given a Kronecker $k$-jet-web $\mathcal{W}$ near $0 \in \mathbb{C}^{n}$, one can find $U \subset$ $\mathbb{C}^{n}, 0 \in U$, and a Kronecker web $\mathcal{W}^{\prime}$ on $U$ such that $k$-jet of $\mathcal{W}^{\prime}$ at 0 coincides with $\mathcal{W}$

Anyway, we proved the following 
Corollary 4.21. Given a Kronecker web $\mathcal{W}$, the total space of the vector bundle $\Phi(\mathcal{W})$ is equipped with a natural bihamiltonian structure.

\section{PAIRS OF SKEW-SYMMETRIC FORMS}

Recall the classification of pairs of skew-symmetric bilinear pairings from [12] (see also $[13,14])$. For $k \in \mathbb{N}$ consider the identity $k \times k$ matrix $I_{k}$. For $\mu \in \mathbb{C}$ consider the Jordan block $J_{k, \mu}$ of size $k$ and eigenvalue $\mu$. The pair of matrices

$$
\mathrm{H}_{1}^{(\mu)}=\left(\begin{array}{cc}
0 & J_{k, \mu} \\
-J_{k, \mu}^{t} & 0
\end{array}\right), \quad \mathrm{H}_{2}^{(\mu)}=\left(\begin{array}{cc}
0 & I_{k} \\
-I_{k} & 0
\end{array}\right)
$$

defines a pair of skew-symmetric bilinear pairings on vector space $\mathbb{C}^{2 k}$. The limit case of $\mu \rightarrow \infty$ may be deformed to

$$
\mathrm{H}_{1}^{(\infty)}=\left(\begin{array}{cc}
0 & I_{k} \\
-I_{k} & 0
\end{array}\right), \quad \mathrm{H}_{2}^{(\infty)}=\left(\begin{array}{cc}
0 & J_{k, 0} \\
-J_{k, 0}^{t} & 0
\end{array}\right) .
$$

Denote the pair $\left(\mathrm{H}_{1}^{(\mu)}, \mathrm{H}_{2}^{(\mu)}\right)$ of skew-symmetric bilinear pairings by $\mathcal{J}_{2 k, \mu}, k \in \mathbb{N}$, $\mu \in \mathbb{C P}^{1}$.

Add to this list the so-called Kroneker pair $\mathcal{K}_{2 k-1}$. This is a pair in a vector space $\mathbb{C}^{2 k-1}$ with a basis $\left(\boldsymbol{w}_{0}, \boldsymbol{w}_{1}, \ldots, \boldsymbol{w}_{2 k-2}\right)$. The only non-zero pairings are

$$
\left(\boldsymbol{w}_{2 l}, \boldsymbol{w}_{2 l+1}\right)_{1}=1, \quad\left(\boldsymbol{w}_{2 l+1}, \boldsymbol{w}_{2 l+2}\right)_{2}=1,
$$

for $0 \leq l \leq k-2$. Obviously, different pairs from this list are not isomorphic.

Theorem 5.1. ([12, 32]) Any pair of skew-symmetric bilinear pairings on a finitedimensional complex vector space can be decomposed into a direct sum of pairs of the pairings isomorphic to $\mathcal{J}_{2 k, \mu}, k \in \mathbb{N}, \mu \in \mathbb{P}^{1}$, and $\mathcal{K}_{2 k-1}, k \in \mathbb{N}$. The types of the components of this decomposition are uniquely determined.

Though this simple statement was known for a long time (for example, the preprint of [32] existed in 1973), we do not know whether it was published before it was used in [12]. The discussions in [9] and [36] come very close, but do not state this result.

Definition 5.2. A pair of bilinear skew-symmetric forms $(,)_{1}$ and $(,)_{2}$ in a vector space $V$ over $\mathbb{C}$ is Kronecker if it has no Jordan blocks. The rank of a Kronecker pair is the number of Kronecker blocks in the decomposition of the pair into indecomposable components. ${ }^{24}$

Given a Kronecker pair of bilinear skew-symmetric forms in $V$ let the action subspace $^{25}$ of $V$ be spanned by vector subspaces $\operatorname{Ker}(,)_{\lambda_{1}, \lambda_{2}},\left(\lambda_{1}, \lambda_{2}\right) \in \mathbb{C}^{2} \backslash(0,0)$. Here $\left(v, v^{\prime}\right)_{\lambda_{1}, \lambda_{2}} \stackrel{\text { def }}{=} \lambda_{1}\left(v, v^{\prime}\right)_{1}+\lambda_{2}\left(v, v^{\prime}\right)_{2}$.

\footnotetext{
${ }^{24}$ Note a conflict in these notations: for a pair $(,)_{1,2}$ of rank $r$, both forms have corank $k$.

${ }^{25}$ The reason for the name is that in applications this subspace is spanned by differentials of action function of a system of action-angle variables.
} 
Proposition 5.3. The action subspace of a Kronecker pair of bilinear forms in $V$ of rank $r$ is isotropic with respect to $(,)_{1}$ and $(,)_{2}$, and has dimension $\frac{\operatorname{dim} V+r}{2}$. It is a maximal isotropic subspace for any form $(,)_{\lambda_{1}, \lambda_{2}},\left(\lambda_{1}, \lambda_{2}\right) \in \mathbb{C}^{2} \backslash(0,0)$.

Proof. This follows immediately from the explicit form of a Kronecker block.

Proposition 5.4. The action subspace $\mathcal{A}$ of a Kronecker pair of bilinear forms in $V$ of rank $r$ has a natural Kronecker linear relation of rank $r$.

Proof. Since $\mathcal{A}$ is isotropic with respect to $(,)_{1},(,)_{1}$ induces a natural pairing of $\mathcal{A}$ with $V / \mathcal{A}$, or a mapping $\alpha_{1}: \mathcal{A} \rightarrow(V / \mathcal{A})^{*}$. Similarly, ()$_{2}$ induces a mapping $\alpha_{2}: \mathcal{A} \rightarrow(V / \mathcal{A})^{*}$. Consider the relation $\alpha_{2}^{-1} \alpha_{1}$ in $\mathcal{A}$. Looking on the explicit form of a Kronecker block of a pair of skew-symmetric bilinear forms, one can easily recognize in the relation $\alpha_{2}^{-1} \alpha_{1}$ a direct sum of Kronecker blocks.

Proposition 5.5. Consider two families $(,)_{1, t},(,)_{2, t}, t \in T$, of skew-symmetric bilinear forms in a vector space $V$, parameterized by a manifold $T$. Suppose that there is $r \in \mathbb{N}$ such that for any $t \in T$ the pair $(,)_{1, t},(,)_{2, t}$ is Kronecker of rank $r$. Let $\mathcal{A}_{t}$ be the action subspace of the pair $(,)_{1, t},(,)_{2, t}$. Then $\mathcal{A}_{t}$ depends smoothly on $t$.

Proof. This follows immediately from the following

Lemma 5.6. Consider a vector space $V$ and numbers $n_{1}, \ldots, n_{k}, N \in \mathbb{N}$. Consider the product $X$ of Grassmannians $\prod_{i=1}^{k} \operatorname{Gr}_{n_{i}}(V)$ and the subset $Z \subset X$ consisting of $k$-tuples of subspaces such that the linear span of such a $k$-tuple has dimension $N$. Consider the mapping

$$
\iota: Z \rightarrow \operatorname{Gr}_{N}(V):\left(V_{1}, \ldots, V_{k}\right) \mapsto V_{1}+\cdots+V_{k},
$$

here $V_{i}$ is a vector subspace of $V$ of dimension $n_{i}$. Then the mapping $\iota$ is smooth.

Proof. Let $x_{0} \in Z, U_{0}$ be a small neighborhood of $x_{0}$ in $X$. For $x \in X$ denote by $V_{i}(x)$ the $i$-th component of $x, V_{i}(x) \in \mathrm{Gr}_{n_{i}}(V)$. Choose a basis $v_{i l}(x)$ in $V_{i}(x)$, $i=1, \ldots, k, l=1, \ldots, n_{i}$, which depends regularly on $x \in U$. Pick up a basis $\left\{v_{\alpha}\left(x_{0}\right)\right\}_{\alpha \in A}$ out of vectors $v_{i l}\left(x_{0}\right), i=1, \ldots, k, l=1, \ldots, n_{i}$ (each $\alpha$ has a form $\left.i l\right)$. Then in an open subset $U_{1}$ of $U_{0}$ the vectors $v_{\alpha}(x)$ remain linearly independent, thus on $U_{1} \cap Z$ they span $V_{1}+\cdots+V_{k}$. This implies ${ }^{26}$ that $V_{1}(z)+\cdots+V_{k}(z)$ depends regularly on $z \in Z$.

This finishes the proof of Proposition 5.5.

\section{Micro-Kronecker bihamiltonian Structures}

Recall that a bihamiltonian structure on $M$ induces a pair $(,)_{1},(,)_{2}$ of bilinear skew-symmetric forms in $\mathcal{T}^{*} M$. As a corollary, it also induces a pair of bilinear skew-symmetric forms in $\mathcal{T}^{*} M \otimes \mathbb{C}$, which we will denote by the same symbols.

\footnotetext{
${ }^{26} \mathrm{~A}$ similar argument can show that $Z$ is a submanifold of $X$.
} 
Definition 6.1. A bihamiltonian structure on $M$ is micro-Kronecker at $m \in M$ if the pair of bilinear skew-symmetric forms $(,)_{1}$ and $(,)_{2}$ in $\mathcal{T}_{m}^{*} M$ has no Jordan blocks. The rank of the bihamiltonian structure at $m \in M$ is the number of Kronecker blocks. A bihamiltonian structure on $M$ is micro-Kronecker of rank $r$ if it is micro-Kronecker at all the points $m \in M$ of the same rank $r$.

The definition above is almost identical to the definition of completeness in [4] (see also [28]), a similar but more restrictive definition of homogeneity was introduced in [11].

Proposition 6.2. Consider a micro-Kronecker bihamiltonian structure of rank $r$ on a manifold $M$. There is a foliation $\mathcal{F}$ on $M$ such that for any $m \in M$ the action subspace of $\mathcal{T}_{m}^{*} M$ is the normal space to the leaf of $\mathcal{F}$ through $m$.

Proof. By Proposition 5.5 action subspaces from a subbundle of $\mathcal{T}^{*} M$. Consider $\left(\lambda_{1}, \lambda_{2}\right) \in \mathbb{K}^{2} \backslash(0,0)$ and the Poisson structure $\lambda_{1}\{,\}_{1}+\lambda_{2}\{,\}_{2}$ on $M$. This Poisson structure has corank $r$, consider its symplectic foliation $\widetilde{\mathcal{F}}_{\lambda_{1}, \lambda_{2}}$. Then the kernel of the bilinear form $(,)_{\lambda_{1}, \lambda_{2}}$ in $\mathcal{T}_{m}^{*} M$ is the normal space to the leaf of $\widetilde{\mathcal{F}}_{\lambda_{1}, \lambda_{2}}$ through $m$. Now the statement follows from the following lemmas:

Lemma 6.3. Consider a pair of skew-symmetric bilinear forms in $V$ which has no Jordan blocks. Suppose that dimensions of all the Kronecker components of $V$ are $\leq$ $k$. If $\left\{\lambda_{i}\right\}, i \in K$, is a finite subset of $\mathbb{K} \mathbb{P}^{1}$ with $k$ elements, then $V=\sum_{i \in K} \operatorname{Ker}_{\lambda_{i}} W$. Proof. Direct corollary of the explicit form of a Kronecker block.

Lemma 6.4. Consider vector subbundles $E, E_{1}, \ldots, E_{k}$ of $\mathcal{T}^{*} M$, such that $E=$ $\sum E_{i}$. Suppose that $E_{i}$ coincides with the normal bundle to a foliation $\mathcal{F}_{i}$ on $M$, $i=1, \ldots, k$. Then there is a foliation $\mathcal{F}$ on $M$ such that $E$ coincides with the normal bundle to $\mathcal{F}$. Leaves of $\mathcal{F}$ can be described as intersections of leaves of foliations $\mathcal{F}_{i}$.

Proof. This follows immediately for the Frobenius integrability criterion (Proposition 4.17).

This finishes the proof of Proposition 6.2.

Obviously, the foliation $\mathcal{F}$ is canonically defined by the bihamiltonian structure. A (most important) particular case of Proposition 6.2 was announced in [28].

Definition 6.5. Given a micro-Kronecker bihamiltonian structure on a manifold $M$, call the foliation $\mathcal{F}$ of Proposition 6.2 the action foliation ${ }^{27}$ of the bihamiltonian structure.

Definition 6.6. Given a micro-Kronecker bihamiltonian structure on $M$ such that the action foliation $\mathcal{F}$ is a fibration, let $\mathcal{B}_{M}$ be the base of this fibration. If $M$ is clear from the context, denote the base by $\mathcal{B}$.

\footnotetext{
${ }^{27}$ In applications given a system of action-angle variables $\left(H_{i}, \varphi_{i}\right)$, the foliation can be written as $H_{i} \equiv$ const $_{i}$.
} 
From now on suppose that the foliation $\mathcal{F}$ is in fact a fibration of $M$ over the base $\mathcal{B}$. One can always achieve this by decreasing $M$.

Theorem 6.7. The base of the action foliation of a micro-Kronecker bihamiltonian structure has a canonically defined structure of a Kronecker web.

Proof. Indeed, consider $m \in M$ and the projection $b$ of $m$ to $\mathcal{B}$. The (co)differential $\left(\left.d b\right|_{m}\right)^{*}$ of the mapping of projection identifies the vector space $\mathcal{T}_{b}^{*} \mathcal{B}$ with the action subspace at $m$. However, by Proposition 5.4 the action subspace at $m$ is equipped with a Kroneker linear relation, thus $\mathcal{T}_{b}{ }^{*} \mathcal{B}$ is equipped with such a relation as well. To show that $\mathcal{B}$ has a structure of a Kronecker preweb, it is enough to show that this relation in $\mathcal{T}_{b}{ }^{*} \mathcal{B}$ does not depend on the choice of the point $m$ over $b$.

Let $m, m^{\prime}$ be two point of $M$ over $b \in \mathcal{B}$. Let $\mathcal{A}_{m}, \mathcal{A}_{m^{\prime}}$ be the action subspaces in $\mathcal{T}_{m}^{*} M$ and $\mathcal{T}_{m^{\prime}}^{*} M$. Both $\mathcal{A}_{m}$ and $\mathcal{A}_{m^{\prime}}$ are identified with $\mathcal{T}_{b}^{*} \mathcal{B}$, thus one with the other.

Lemma 6.8. For any $\lambda \in \mathbb{K} \mathbb{P}^{1}$ the identification between $\mathcal{A}_{m}$ and $\mathcal{A}_{m^{\prime}}$ sends $\operatorname{Ker}_{\lambda} W_{m} \subset$ $\mathcal{A}_{m}$ to $\operatorname{Ker}_{\lambda} W_{m^{\prime}} \subset \mathcal{A}_{m^{\prime}}$.

Proof. Let $\lambda=\lambda_{1}: \lambda_{2}$. Consider the Poisson structure $\lambda_{1}\{,\}_{1}+\lambda_{2}\{,\}_{2}$ on $M$. Then $\operatorname{Ker}_{\lambda} W$ is the normal bundle to the symplectic foliation $\widetilde{\mathcal{F}}_{\lambda}$ for this Poisson structure. Consider the leaf $L$ of this foliation which passes through $m$. This leaf contains the leaf of the action foliation $\mathcal{F}$ through $m$, thus $L$ is a preimage of a submanifold $\widetilde{L} \subset \mathcal{B}$. Thus $\operatorname{Ker}_{\lambda} W_{m} \subset \mathcal{A}_{m}$ is the image of the normal space to $\widetilde{L}$ under the (co)differential of the projection mapping.

By the same reason $m^{\prime}$ is in $L$, and $\operatorname{Ker}_{\lambda} W_{m^{\prime}} \subset \mathcal{A}_{m^{\prime}}$ is also the image the same normal space. Thus the identification of $\mathcal{A}_{m}$ and $\mathcal{A}_{m^{\prime}}$ via projection to $\mathcal{B}$ indeed sends $\operatorname{Ker}_{\lambda} W_{m}$ to $\operatorname{Ker}_{\lambda} W_{m^{\prime}}$.

Lemma 6.9. Consider vector spaces $V$ and $V^{\prime}$ with Kronecker linear relations $W$ and $W^{\prime}$ in $V$ and $V^{\prime}$ correspondingly. Consider an isomorphism $\alpha: V \rightarrow V^{\prime}$. If $\alpha$ sends $\operatorname{Ker}_{\lambda} W$ to $\operatorname{Ker}_{\lambda} W^{\prime}$ for any $\lambda \in \mathbb{K P}^{1}$, then $\alpha \oplus \alpha$ sends $W \subset V \oplus V$ to $W^{\prime} \subset V^{\prime} \oplus V^{\prime}$.

This lemma is equivalent to Theorem 7.1 proven in Section 7 .

We had shown that the base $\mathcal{B}$ of the action foliation has a canonically defined structure of a Kronecker preweb. Denote by $\widetilde{\mathcal{W}}_{b}$ the relation in $\mathcal{T}_{b}^{*} \mathcal{B}, b \in \mathcal{B}$.

To show that this preweb is in fact a web, it is enough to describe the $\lambda$-integrating foliation $\mathcal{F}_{\lambda}$ of $\widetilde{\mathcal{W}}$. Fix $\lambda_{1}, \lambda_{2} \in \mathbb{K}$, consider the Poisson structure $\lambda_{1}\{,\}_{1}+\lambda_{2}\{,\}_{2}$ on $M$. Since this is a Poisson structure of constant rank, symplectic leaves form a foliation on $M$. This foliation depends only on $\lambda_{1}: \lambda_{2} \in \mathbb{K P}^{1}$, denote this foliation $\widetilde{\mathcal{F}}_{\lambda_{1}: \lambda_{2}}$.

The normal space to this foliation at $m \in M$ is the kernel of the bilinear form $\lambda_{1}(,)_{1}+\lambda_{2}(,)_{2}$ in $\mathcal{T}_{m}^{*} M$. In particular, the normal bundle to $\widetilde{\mathcal{F}}_{\lambda_{1}: \lambda_{2}}$ is contained in the action bundle of the bihamiltonian structure. Thus $\mathcal{F}$ is a subfoliation of 
$\widetilde{\mathcal{F}}_{\lambda_{1}: \lambda_{2}}$. In particular, $\widetilde{\mathcal{F}}_{\lambda_{1}: \lambda_{2}}$ induces a "quotient" foliation $\mathcal{F}_{\lambda_{1}: \lambda_{2}}$ on the base $\mathcal{B}$ of the foliation $\mathcal{F}$. Now one can immediately see that the normal space to the foliation $\mathcal{F}_{\lambda_{1}: \lambda_{2}}$ at $b \in \mathcal{B}$ coincides with $\operatorname{Ker}_{\lambda} \widetilde{\mathcal{W}}_{b}$, thus $\mathcal{F}_{\lambda_{1}: \lambda_{2}}$ is the $\lambda_{1}: \lambda_{2}$-integrating foliation of the preweb on $\mathcal{B}$. Thus the preweb structure on $\mathcal{B}$ is indeed a Kronecker web.

A statement which is parallel to a particular case of Theorem 6.7 was announced in [28].

\section{LATTICE OF KERNELS}

Given a bisurjective relations $W$ in $V$, one obtains a collection of vector subspaces $\operatorname{Ker}_{\lambda} W, \lambda \in \mathbb{K} \mathbb{P}^{1}$, of the vector space $V$. The goal of this section is to prove

Theorem 7.1. Let $G R(V)$ denote the set of all vector subspaces of a vector space $V$. Associate to a bisurjective linear relation $W$ in $V$ a mapping of sets $\mathcal{K}_{W}: \mathbb{K} \mathbb{P}^{1} \rightarrow$ $G R(V), \mathcal{K}_{W}(\lambda)=\operatorname{Ker}_{\lambda} W$.

If $W$ and $W^{\prime}$ are two bisurjective linear relations in $V$ such that $\mathcal{K}_{W}=\mathcal{K}_{W^{\prime}}$ and $W$ is Kronecker, then $W=W^{\prime}$.

This theorem is a corollary of the following

Amplification 7.2. In the conditions of Theorem 7.1 suppose that all the Kronecker blocks of $W$ have dimensions $k$ or less. Let $\Lambda \subset \mathbb{K P}^{1}$ be a collection of $k+1$ points. Then if $\left.\mathcal{K}_{W}\right|_{\Lambda}=\left.\mathcal{K}_{W^{\prime}}\right|_{\Lambda}$, then $W=W^{\prime}$.

Proof (V. Serganova). Given a bisurjective relation $\boldsymbol{W}$ in a vector space $\boldsymbol{V}$, suppose that $\boldsymbol{K}=\operatorname{Ker}_{\lambda_{1}: \lambda_{2}} \boldsymbol{W}$. Define the lifting $\boldsymbol{K}^{(2)}$ of $\boldsymbol{K}$ into $\boldsymbol{V} \times \boldsymbol{V}$ as the collection of vectors $\left\{\left(\lambda_{1} k, \lambda_{2} k\right) \subset \boldsymbol{V} \times \boldsymbol{V} \mid k \in \boldsymbol{K}\right\}$. One can easily check that $\boldsymbol{K}^{(2)} \subset \boldsymbol{W}$.

Now suppose that $\boldsymbol{W}$ is Kroneker. Let $\Lambda=\left\{\lambda_{1}, \ldots, \lambda_{k+1}\right\} \subset \mathbb{K} \mathbb{P}^{1}, \boldsymbol{K}_{i}=\operatorname{Ker}_{\lambda_{i}} \boldsymbol{W}$, and consider $\boldsymbol{K}_{i}^{(2)} \subset \boldsymbol{W}$. It is sufficient to show that these vector subspaces span $\boldsymbol{W}$. Indeed, assume that this is true. Then the statement of the amplification is obvious if $W^{\prime}$ is Kronecker. In general, in the conditions of the amplification let $V=V_{\text {Kron }} \oplus V_{\text {Jord }}$; here $V_{\text {Kron }}$ is a sum of Kroneker blocks of $W^{\prime}, V_{\text {Jord }}$ is a sum of Jordan blocks of $W$. Let $W_{\text {Kron }}^{\prime}=W^{\prime} \cap\left(V_{\text {Kron }} \oplus V_{\text {Kron }}\right)$ be restriction of $W^{\prime}$ to $V_{\text {Kron }}$. We know that vector subspaces $\boldsymbol{K}_{i}^{(2)}$ span $W$, that $\boldsymbol{K}_{i}^{(2)}$ are subspaces of $W^{\prime}$, and that $W_{\text {Kron }}^{\prime}$ is spanned by some subspaces of $\boldsymbol{K}_{i}^{(2)}$, thus $W_{\text {Kron }}^{\prime} \subset W \subset W^{\prime}$. If $n$, $n^{\prime}$ are the numbers of Kronecker blocks in $W$ and $W_{\text {Kron }}^{\prime}$, then $\operatorname{dim} W=\operatorname{dim} V+n$ and $\operatorname{dim} W^{\prime}=\operatorname{dim} V+n^{\prime}$. Hence $n^{\prime} \geq n$. On the other hand, $n=\operatorname{dim}_{n^{\prime}} \operatorname{Ker}_{\lambda} W$, $n^{\prime}=\operatorname{dim} \operatorname{Ker}_{\lambda} W_{\text {Kron }}^{\prime} \leq \operatorname{dim} \operatorname{Ker}_{\lambda} W^{\prime}=\operatorname{dim}_{K^{\prime}} W=n$. Thus $n^{\prime} \leq n$. Hence $\operatorname{dim} W=\operatorname{dim} W^{\prime}$, thus $W=W^{\prime}$.

What remains to prove is that that vector subspaces $\boldsymbol{K}_{i}^{(2)} \subset \boldsymbol{W}$ span $\boldsymbol{W}$ if $\boldsymbol{W}$ is Kronecker. Decomposing into a direct sum, we can restrict our attention to the case when $\boldsymbol{W}$ is one Kroneker block of dimension $k$ or less. By decreasing $\Lambda$ we may assume that $\operatorname{dim} \boldsymbol{V}=k$. Then $\operatorname{dim} \boldsymbol{W}=k+1, \operatorname{dim} \boldsymbol{K}_{i}=\operatorname{dim} \boldsymbol{K}_{i}^{(2)}=1$ for any $i$. Thus it is enough to show that $\boldsymbol{K}_{i}^{(2)}, i=1, \ldots, k+1$, are linearly independent. 
Since all the Kroneker blocks of dimension $k$ are isomorphic, it is enough to do this for one particular Kroneker block of dimension $k$. Consider a 2-dimensional vector space $\mathcal{S}$ with basis $\boldsymbol{s}_{1}, \boldsymbol{s}_{2}$ (as in Definition 2.2). Let $\boldsymbol{V}$ be the symmetric power $\operatorname{Sym}^{k-1} \mathcal{S}$. Consider $\boldsymbol{V}$ as a subspace of the vector space of polynomials on $\mathcal{S}^{*}$, then two partial derivatives $\partial_{1}, \partial_{2}$ define two mappings $V=\operatorname{Sym}^{k-1} \mathcal{S} \rightarrow \operatorname{Sym}^{k-2} \mathcal{S}$. Let $\boldsymbol{W} \subset \boldsymbol{V} \times \boldsymbol{V}$ consists of pairs $\left(v_{1}, v_{2}\right)$ such that $\partial_{1} v_{1}=\partial_{2} v_{2}$. One can momentarily check that $\boldsymbol{W}$ is a Kronecker block in $\boldsymbol{V}$.

Obviously, $\operatorname{Ker}_{\lambda_{1}: \lambda_{2}} \boldsymbol{W}$ is spanned by $\left(\lambda_{1} \boldsymbol{s}_{2}-\lambda_{2} \boldsymbol{s}_{1}\right)^{k-1} \subset \boldsymbol{V}$. Let $\boldsymbol{V}^{\prime}=\mathrm{Sym}^{k} \mathcal{S}$, introduce a mapping $\boldsymbol{V} \times \boldsymbol{V} \stackrel{\alpha}{\rightarrow} \boldsymbol{V}^{\prime}$ given by $\left(p, p^{\prime}\right) \mapsto \boldsymbol{s}_{2} p-\boldsymbol{s}_{1} p^{\prime}$. One can easily check that $\alpha\left(\left(\operatorname{Ker}_{\lambda_{1}: \lambda_{2}} W\right)^{(2)}\right)$ is spanned by the vector $\left(\lambda_{1} \boldsymbol{s}_{2}-\lambda_{2} \boldsymbol{s}_{1}\right)^{k} \subset \boldsymbol{V}^{\prime}$. Thus to show that $\boldsymbol{K}_{i}^{(2)}$ are linearly independent, it is enough to show that for $k+1$ non-proportional linear functions $l_{1}, \ldots, l_{k+1}$ on $\mathcal{S}^{*}$ the polynomials $l_{1}^{k}, \ldots, v_{k+1}^{k}$ are linearly independent. In turn, this is an obvious corollary of non-vanishing of Vandermont determinant.

Later we will need the following lemma, which in the Kronecker case is a corollary of the proof, and in Jordan case follows immediately from the explicit description of Jordan blocks:

Lemma 7.3. Consider a relation $W \subset V \oplus V$ which is a Kronecker block with $\operatorname{dim} V \leq k$, or is a Jordan block. Let $\left\{\lambda_{i}\right\}, 1 \leq i \leq k$, be a subset of $\mathbb{K} \mathbb{P}^{1}$. Then $\operatorname{Ker}_{\lambda_{k}} W \cap \sum_{i=1}^{k-1} \operatorname{Ker}_{\lambda_{i}} W=0$.

\section{LAGRANGIAN FOLIATIONS}

Here we recall more or less standard results of symplectic geometry which will be useful in Section 9. See [3] for details.

Definition 8.1. Given a bracket $\{$,$\} on M$, a submanifold $L \subset M$ is involutive if $\left.\{f, g\}\right|_{L}=0$ for any functions $f$ and $g$ on $M$ such that ${ }^{28}$ both $\left.f\right|_{L}$ and $\left.g\right|_{L}$ are constant. Call $L$ Lagrangian if it is involutive, and any submanifold $L_{1} \subset L$ of codimension 1 or more is not involutive. A foliation $\mathcal{F}$ on $M$ is Lagrangian if each leaf of $\mathcal{F}$ is Lagrangian.

In the context of Example 0.6 the foliation on fibers of the projection $\pi$ is Lagrangian. The 0 -section of $\pi$ is a Lagrangian submanifold. In fact, on a symplectic manifold $M$ any Lagrangian submanifold $L$ has $\operatorname{dim} L=\frac{\operatorname{dim} M}{2}$, an involutive submanifold is Lagrangian if it has this dimension, and locally any Lagrangian foliation can be reduced to the foliation on fibers of the projection $\pi$ of Example 0.6:

Definition 8.2. A locally-affine structure on a manifold $L$ is a connection in $\mathcal{T} L$ with vanishing curvature and torsion.

\footnotetext{
${ }^{28}$ In complex-analytic situation one needs to consider functions on open subsets of $M$.
} 
Proposition 8.3. Consider a symplectic Poisson structure on $M$, and a Lagrangian foliation $\mathcal{F}$. Suppose that leaves of $\mathcal{F}$ are fibers ${ }^{29}$ of a projection $\pi: M \rightarrow N$. Let $L$ be a leaf of the foliation $\mathcal{F}, \pi(L)=\{n\} \subset N$. Then there is a canonical identification of $\mathcal{T}_{l} L$ with with $\mathcal{T}_{n}^{*} N$ for any $l \in L$, thus all the tangent spaces to $L$ can be identified with each other. This identification provides $L$ with a locally-affine structure.

Consider a section $s: N \rightarrow M$ of the projection $\pi$. If the image $\operatorname{Im} s \subset M$ is a Lagrangian submanifold, there is exactly one identification of a neighborhood of $\operatorname{Im} s$ with an open subset of $\mathcal{T}^{*} N$ which is compatible with the projections on $N$, with Poisson structures on $M$ and $\mathcal{T}^{*} N$, and which sends $\operatorname{Im} s$ to the 0 -section of $\mathcal{T}^{*} N$.

The identification above sends the locally-affine structure on leaves of $\mathcal{F}$ to the tautological locally-affine structure on vector spaces $\mathcal{T}_{n} N, n \in N$.

Remark 8.4. Obviously, a locally-affine structure on a contractible set is isomorphic to the tautological connection in $\mathcal{T} U$; here $U$ is an appropriate open subset of a vector space. Thus another way to define a locally affine structure is to introduce identifications of open subsets $U_{i}$ which cover $L$ with open subsets of vector spaces, and require that the transition functions on $U_{i} \cap U_{j}$ correspond to affine mappings of these vector spaces.

Definition 8.5. Consider a small open connected subset $U$ of a manifold $L$ with a locally-affine structure. Then tangent spaces at different points $l \in U$ are identified with each other (such an identification may depend on a choice of homotopy type of a curve which connects these points). Call an open subset $U \subset L$ simple if the identifications above do not depend on the choice of homotopy class of connecting curves. If $U$ is simple, the corresponding set of equivalence classes of tangent vectors has a vector space structure. Call this structure the vector space associated to a simple locally-affine structure on $U$.

Corollary 8.6. Consider a Lagrangian foliation $\mathcal{F}$ on a symplectic manifold. Let $B$ be a local base of $\mathcal{F}$. By Proposition 8.3 leaves of $\mathcal{F}$ have a canonical locally-affine structure. The vector space associated to the leaf over $b \in B$ is canonically identified with $\mathcal{T}_{b}^{*} B$.

We will need an explicit construction of the identification of $\mathcal{T}_{m} L$ with $\mathcal{T}_{n}^{*} N, n=$ $\pi(m)$, from Proposition 8.3. Given a Lagrangian submanifold $L$ in a symplectic manifold $M$, the Hamiltonian mapping $\mathrm{H}: \mathcal{T}_{m}^{*} M \rightarrow \mathcal{T}_{m} M$ can be restricted to a mapping $\mathcal{N}_{m}^{*} L \rightarrow \mathcal{T}_{m} L, m \in L$, which is a bijection. On the other hand, for any leaf $L$ of any foliation there is a canonical flat connection on the normal bundle $\mathcal{N} L$ to $L$, thus on the dual bundle $\mathcal{N}^{*} L$. Identifying $\mathcal{N}^{*} L$ with $\mathcal{T} L$, one obtains a flat connection on $\mathcal{T} L$.

Moreover, $\mathcal{N}_{m}^{*} L$ is identified with $\mathcal{T}_{\pi(m)}^{*} N$. Basing on these data, it is easy to construct the identification of Proposition 8.3.

Now extend this discussion to the case of Poisson structures of constant rank.

\footnotetext{
${ }^{29}$ Locally any foliation can be represented in such a form.
} 
Proposition 8.7. Any Lagrangian submanifold of a Poisson manifold $M$ is contained in a symplectic leaf $f^{30}$ of $M$. If the Poisson structure on $M$ is of constant rank, Lagrangian foliations on $M$ coincide with smooth families of Lagrangian foliations, one per each symplectic leaf of $M$.

Remark 8.8. If the Lagrangian foliation is a fibration $M \stackrel{\pi}{\rightarrow} B$, and Poisson structure on $M$ is of constant rank, each symplectic leaf $S \subset M$ coincides with $\pi^{-1} \pi S$, thus the symplectic foliation $\widetilde{\mathcal{F}}$ on $M$ is a preimage of a foliation $\mathcal{F}_{0}$ on $B$. Due to Proposition 8.3, given a section $s$ of $\pi$ such that intersection of $\operatorname{Im} s$ with each symplectic leaf $S$ is Lagrangian in $S$, one can identify a neighborhood of $\operatorname{Im} s$ with a neighborhood of the 0 -section of $\mathcal{T}^{*} \mathcal{F}_{0}$. Moreover, $\mathcal{T}^{*} \mathcal{F}_{0}$ carries a natural Poisson structure, and the identification above is compatible with Poisson structures on $\mathcal{T}^{*} \mathcal{F}_{0}$ and $M$.

\section{TWO FUNCTORS}

Presently we have two constructions: by Corollary 4.21, given a Kronecker web $\mathcal{W}$ on $B$ of rank $r$, one can construct a bihamiltonian structure on the total space of $\Phi(\mathcal{W})$ (which has dimension $2 \operatorname{dim} B-r$ ). By Theorem 6.7, given a micro-Kronecker bihamiltonian structure $M$ of dimension $d$ and rank $r$, one can associate to small open subsets $U \subset M$ a Kronecker web structure on manifold $\mathcal{B}_{U}$ (of dimension $\frac{d+r}{2}$ ). Investigate the relation of these two constructs.

Proposition 9.1. Consider a Kronecker web $\mathcal{W}$ of rank $r$ on a manifold $B$.

1. If $\left(\lambda_{1}, \lambda_{2}\right) \in \mathbb{K}^{2} \backslash(0,0)$, then the symplectic foliation of the Poisson bracket $\lambda_{1}\{,\}_{1}+\lambda_{2}\{,\}_{2}$ on $\Phi(\mathcal{W})$ is the preimage of the integrating foliation $\mathcal{F}_{\lambda_{1}: \lambda_{2}}$ on $B$.

2. The bihamiltonian structure on $\Phi(\mathcal{W})$ is micro-Kronecker of rank $r$.

3. The action foliation on $\Phi(\mathcal{W})$ coincides with foliation on fibers of projection $\Phi(\mathcal{W}) \rightarrow B$.

4. The structure of Kronecker web on $B$ induced by the bihamiltonian structure on $\Phi(\mathcal{W})$ coincides with the initial Kronecker web structure $\mathcal{W}$ on $B$.

Proof. The first statement follows from the definition of the Poisson bracket $\{,\}_{\lambda_{1}: \lambda_{2}}$ on $\Phi(\mathcal{W})$. If $\mathbb{K}=\mathbb{C}$, the second statement is a direct corollary of the first one. If $\mathbb{K}=\mathbb{R}$, the second statement follows from the following

Lemma 9.2. Consider a pair of skew-symmetric bilinear forms $(,)_{1}$ and $(,)_{2}$ in a vector space $V$. Then $\operatorname{dim} \operatorname{Ker}\left(\lambda_{1}(,)_{1}+\lambda_{2}(,)_{2}\right)$ is constant for $\left(\lambda_{1}, \lambda_{2}\right)$ inside an open subset of $\mathbb{K}^{2}$, call this common value $r$. Suppose that for any finite subset $\Lambda_{0} \subset \mathbb{K} \mathbb{P}^{1}$ there is $\Lambda \subset \mathbb{K} \mathbb{P}^{1} \backslash \Lambda_{0}$ such the vector subspaces $\operatorname{Ker}\left(\lambda_{1}(,)_{1}+\lambda_{2}(,)_{2}\right)$, $\lambda_{1}: \lambda_{2} \in \Lambda$, span a vector subspace of $V$ of dimension $\frac{\operatorname{dim} V+r}{2}$. Then the pair $(,)_{1}$, $(,)_{2}$ is Kronecker.

\footnotetext{
${ }^{30}$ It is possible to define what is a symplectic leaf of an arbitrary Poisson structure. However, for the purpose of our discussion, it is enough to restrict attention to Poisson structures of constant rank, as in Remark 0.13.
} 
Proof. Lemma follows immediately from the classification of Theorem 5.1.

The remaining statements of the proposition follow immediately from the first two statements.

Proposition 9.3. Consider a micro-Kronecker bihamiltonian structure on a manifold $M$. Then

1. the fibers of action foliations have a natural locally-affine structure;

2. suppose that the action foliation is a fibration with a base $\mathcal{B}$, and the locallyaffine structures on fibers are simple. Denote by $E$ the vector bundle over $\mathcal{B}$ which is associated ${ }^{31}$ to the bundle of locally-affine structures mentioned above. This vector bundle is canonically isomorphic to $\Phi(\mathcal{B})$.

Proof. Consider Hamiltonian mappings $\mathrm{H}_{1,2}: \mathcal{T}_{m}^{*} M \rightarrow \mathcal{T}_{m} M$ of Poisson structures on $M$. Restrict these mappings to the action subspace $\mathcal{A}_{m} \subset \mathcal{T}_{m}^{*} M$. By Proposition 5.3 the action subspace is isotropic with respect to both pairings in $\mathcal{T}_{m}^{*} M$, thus the elements $\mathrm{H}_{1,2} a, a \in \mathcal{A}_{m}$, are orthogonal ${ }^{32}$ to $\mathcal{A}_{m}$. By definition, $\mathcal{A}_{m}^{\perp}$ coincides with the tangent space to the fiber $L_{m}$ of the action foliation which passes through $m$. In particular, there are two mappings $\widetilde{\mathrm{H}}_{1,2}: \mathcal{A}_{m} \rightarrow \mathcal{T}_{m} L_{m}$. Note that these mappings form a pencil which corresponds to the linear relation in $\mathcal{A}_{m}$ (as in Proposition 5.4 and in the proof of Theorem 6.7).

Since the statement of the proposition is local on $M$, one can suppose that the action foliation is a fibration. Let $\pi: M \rightarrow \mathcal{B}$ be the projection to the base of foliation, and $b=\pi(m)$. Then $\pi^{*}$ induces a canonical isomorphism $\mathcal{T}_{b}{ }^{*} \mathcal{B} \simeq \mathcal{A}_{m}$. Now $\mathcal{B}$ is a Kronecker web, thus $\mathcal{T}_{b}{ }^{*} \mathcal{B}$ carries a Kronecker relation, and this relation is compatible with the relation on $\mathcal{A}_{m}$ w.r.t. the isomorphism above. On the other hand, let $\Phi_{b}$ be the fiber of $\Phi(\mathcal{B})$ over $b$. Then the linear relation in $\mathcal{T}_{b}{ }^{*} \mathcal{B}$ can be described both by a pencil $\widetilde{\mathcal{P}}_{1,2}=\widetilde{\mathrm{H}}_{1,2} \circ \pi^{*}: \mathcal{T}_{b}{ }^{*} \mathcal{B} \rightarrow \mathcal{T}_{m} L_{m}$ and by a pencil $\mathcal{P}_{1,2}: \mathcal{T}_{b}^{*} \mathcal{B} \rightarrow \Phi_{b}$. Proposition 2.4 gives a canonical isomorphism between $\mathcal{T}_{m} L_{m}$ and $\Phi_{b}, b=\pi(m)$. As a corollary there is a canonical identification of tangent spaces to $L_{m}$ at different points (since they all project to the same point of $\mathcal{B}$ ). This induces a flat connection $\nabla$ on the tangent bundle to $L_{m}$.

What remains is to show that this connection on $L_{m}$ is a locally-affine structure. Pick up any $\lambda \in \mathbb{P}^{1}$, for example, $\lambda=1$ : 0 . Consider the corresponding Poisson structure $1\{,\}_{1}+0\{,\}_{2}$ of the pencil.

By Proposition 5.3, $\left\{\pi^{*} \varphi_{1}, \pi^{*} \varphi_{2}\right\}_{1}=0$ for any functions $\varphi_{1}, \varphi_{2}$ on $\mathcal{B}$. This implies that fibers of $\mathcal{F}$ are involutive submanifolds of $M$ w.r.t. $\{,\}_{1}$. On the other hand, if the rank of bihamiltonian structure on $M$ is $r$, then symplectic leaves of $M$ have dimension $\operatorname{dim} M-r$, and $L_{m}$ has dimension $\frac{\operatorname{dim} M-r}{2}$. Since $L_{m}$ has half the dimension of the symplectic leaf, and is involutive, it is Lagrangian. Thus $\mathcal{F}$ is a Lagrangian

\footnotetext{
${ }^{31}$ As in Definition 8.5.

${ }^{32}$ W.r.t. the standard duality between $\mathcal{T}_{m}^{*} M$ and $\mathcal{T}_{m} M$.
} 
foliation w.r.t. $\{,\}_{1}$. Now Propositions 8.3 and 8.3 imply that the leaves of $\mathcal{F}$ are equipped with canonical locally-affine structures.

Thus it is enough to prove that the connection $\nabla$ on $L_{m}$ constructed above coincides with the connection of this locally-affine structure. Recall that the connection $\nabla$ on $L_{m}$ is constructed basing on the mappings $\widetilde{\mathcal{P}}_{1}: \mathcal{T}_{b}^{*} \mathcal{B} \rightarrow \mathcal{T}_{m} L_{m}$, in other words, on the isomorphisms $\mathcal{T}_{b}{ }^{*} \mathcal{B} / \operatorname{Ker}\left(\widetilde{\mathcal{P}}_{1}\right) \simeq \mathcal{T}_{m} L_{m}$ for different $m$ with $\pi(m)=b$. However, $\mathcal{T}_{b}^{*} \mathcal{B} / \operatorname{Ker}\left(\widetilde{\mathcal{P}}_{1}\right)$ coincides with $\mathcal{T}_{b}{ }^{*} F_{b}$; here $F_{b}$ is the fiber of integrating foliation $\mathcal{F}_{1: 0}$ which passes through $b \in \mathcal{B}$. Let $\widetilde{F}=\pi^{-1} F_{b}$, recall that $\widetilde{F}$ is a symplectic leaf of the Poisson structure $\{,\}_{1}$ on $M$. The foliation $\mathcal{F}$ allows a restriction $\left.\mathcal{F}\right|_{\widetilde{F}}$ to $\widetilde{F}$, this restriction is a Lagrangian foliation on a symplectic manifold. Both the identification $\mathcal{T}_{b}^{*} F_{b}=\mathcal{T}_{b}^{*} \mathcal{B} / \operatorname{Ker}\left(\widetilde{\mathcal{P}}_{1}\right) \simeq \mathcal{T}_{m} L_{m}$ and the identification of $\mathcal{T}^{*} N$ with $\mathcal{T}_{m} L$ in Section 8 are constructed as restrictions of Hamiltonian mappings. Setting $N=F_{b}, L=L_{m}$, and replacing $\mathcal{F}$ with $\left.\mathcal{F}\right|_{\widetilde{F}}$ shows that the connection $\left.\nabla\right|_{\widetilde{F}}$ coincides with the connection of the locally-affine structure of Section 8. This finishes the proof.

Remark 9.4. In the proof above we worked with two locally-affine structures on $L_{m}$ : one constructed basing on the bihamiltonian structure, another based on the Poisson structure $\{,\}_{1}$. However, one could also consider another Poisson structure $\lambda_{1}\{,\}_{1}+$ $\lambda_{2}\{,\}_{2}$. The fact that two locally-affine structures of the proof coincide shows that the locally-affine structures on $L_{m}$ which correspond to the Poisson structure of the pencil $\lambda_{1}\{,\}_{1}+\lambda_{2}\{,\}_{2}$ do not depend on $\left(\lambda_{1}, \lambda_{2}\right) \neq(0,0)$.

\section{Conjecture on Classification}

Conjecture 10.1. Consider a micro-Kronecker bihamiltonian structure on a manifold $M$. Suppose that the restriction of the action foliation to $U_{0} \subset M$ is a fibration with a base $\mathcal{B}_{U_{0}}, m \in U_{0}$, and $b$ is the projection of $m$ to $\mathcal{B}$. Let $\mathcal{W}$ be the Kronecker web on $\mathcal{B}_{U_{0}}$ induced by the bihamiltonian structure on $M$. Let $m^{\prime} \in \Phi(\mathcal{W})$ be the point on the 0 -section of $\Phi(\mathcal{W})$ over $b$. Then there is a local isomorphism of bihamiltonian structures on $M$ near $m$ and on $\Phi(\mathcal{W})$ near $m^{\prime}$.

This conjecture is a generalization of a conjecture from [11]. The case of analytic bihamiltonian structure on $M$ of rank 1 is claimed in [13], the $C^{\infty}$-case of rank 1 is claimed in [34] and proven in [35].

It looks like this conjecture would easily imply all the conjectures of [11]. (Compare with the way we prove Theorem 14.22.)

Remark 10.2. The conjecture implies that to check existence of a local isomorphism between two micro-Kronecker bihamiltonian structures on $M$ and $M^{\prime}$, it is enough to check existence of a local isomorphism between structures of Kronecker webs on local bases of action foliations. By Amplification 7.2 to check an isomorphism of 
Kronecker webs on $B$ and $B^{\prime}$ it is enough to restrict attention to a finite number of foliations on $B$ and $B^{\prime}$. It so happens that for webs which appear in problems of mathematical physics the latter problem is very easy (much easier than for generic Kronecker webs).

In what follows we establish a particular case of the conjecture, and in Section 14 we use this particular case to show flatness of some bihamiltonian systems. After showing that the conjecture is applicable to these systems, the only thing which remains to be proven is that the structure of the Kronecker web on the base of the action foliation is flat, i.e., locally isomorphic to a translation-invariant Kronecker web.

Remark 10.3. Yet another reformulation of the conjecture is that to check that there is a local isomorphism between two micro-Kronecker bihamiltonian structures on $M$ and $M^{\prime}$ one should concentrate attention on action variables (or first integrals) of these structures, and one can completely ignore the question of angle variables. Note that this enormously simplifies the question: for example, usually first integrals may be explicitly written as polynomials in appropriate coordinates, while all one can say about phase variables is that they satisfy some partial differential equations, and that they may be expressed in terms of $\vartheta$-functions.

In this remark we used the following relationship between action variables (i.e., functions which are constant on fibers of action foliation) and first integrals (i.e., particular coordinate functions on a bihamiltonian manifold which arise when one integrates the system): for a micro-Kronecker bihamiltonian system first integrals obtained by Lenard scheme can be taken as action variables. For details see [11].

We prefer an abstract consideration of the (invariantly-defined) action foliation to the language of first integrals, which (at least a priori) may depend on the choice of the particular scheme of integration.

\section{Anti-InVOlutions}

Definition 11.1. An involution is a mapping $f: X \rightarrow X$ such that $f \circ f=$ id. An involution $\alpha: M \rightarrow M$ is an antiinvolution of a Poisson structure $\{$,$\} on a manifold$ $M$ if $\left\{\alpha^{*} f, \alpha^{*} g\right\}=-\alpha^{*}\{f, g\}$ for any two functions $f, g$ on $M$. An antiinvolution of a bihamiltonian structure on a manifold $M$ is a mapping $M \rightarrow M$ which is an anti-involution of both Poisson brackets $\{,\}_{1},\{,\}_{2}$.

Proposition 11.2. Let $\mathcal{W}$ be a Kronecker web on a manifold $B$. The bihamiltonian structure on $\Phi(\mathcal{W})$ allows an anti-involution.

Proof. $\Phi(\mathcal{W})$ is a vector bundle over $B$. Let $\alpha$ be the multiplication by -1 in this bundle. To show that $\alpha$ is an anti-involution, note that $\{,\}_{1}$ is isomorphic to the Poisson structure on $\mathcal{T}^{*} \mathcal{F}_{1: 0}$ (here $\mathcal{F}_{1: 0}$ is the integrating foliation on $B$ ), and this isomorphism is a mapping of vector bundles. Thus it is enough to show that multiplication by -1 is an anti-involution on the cotangent bundle to a foliation. In 
turn, it is enough to prove this for the cotangent bundle to a manifold, which can be checked immediately in local coordinates.

Consider an anti-involution $\iota$ of a micro-Kronecker bihamiltonian structure on a manifold $M$. Let $m \in M$ be a fixed point of $\iota$. There is an $\iota$-invariant open subset $U \ni m$ such that the restriction of the action foliation $\mathcal{F}$ on $U$ is a fibration, denote the base of this fibration by $\mathcal{B}$. Since the action foliation is determined by the set of linear combinations $\lambda_{1}\{,\}_{1}+\lambda_{2}\{,\}_{2}$ of Poisson structures, and not by the parameterization $\left(\lambda_{1}, \lambda_{2}\right)$ of this set, $\iota$ sends leaves of $\mathcal{F}$ to leaves of $\mathcal{F}$. Thus $\iota$ induces an involution $\iota_{\mathcal{B}}$ of $\mathcal{B}$. Consider the submanifold ${ }^{33} \operatorname{Fix}\left(\iota_{\mathcal{B}}\right) \subset \mathcal{B}$ of fixed points of $\iota_{\mathcal{B}}$.

Definition 11.3. The defect of anti-involution $\iota$ at the fixed point $m \in M$ is the codimension of Fix $\left(\iota_{\mathcal{B}}\right) \subset \mathcal{B}$ at the image $b \in \mathcal{B}$ of $m$.

Note that the defect is locally constant on Fix $(\iota)$.

Obviously, the anti-involution of Proposition 11.2 has defect 0 . Now Conjecture 10.1 immediately implies

Conjecture 11.4. Consider a micro-Kronecker bihamiltonian structure on a manifold $M, m \in M$. There is a neighborhood $U \ni m$ and an anti-involution $\alpha$ of the bihamiltonian structure on $U$ such that $\alpha(m)=m$ and the defect of $\alpha$ at $m$ is 0 .

Anti-involutions with defect 0 are important for us because of the following

Theorem 11.5. Suppose that a bihamiltonian structure on a manifold $M$ allows an anti-involution $\iota$ such that $\iota(m)=m, m \in M$, and the defect of $\iota$ at $m$ is 0 . Then Conjecture 10.1 holds for $m$ and $M$.

The theorem is an immediate corollary of Propositions 11.7, 11.8 below.

Definition 11.6. Consider a micro-Kronecker bihamiltonian structure on $M$ with the action foliation $\mathcal{F}$. A submanifold $S \subset M$ is a cross-section if $S$ is a transversal section of foliation $\mathcal{F}$, and for any $\left(\lambda_{1}, \lambda_{2}\right) \in \mathbb{K}^{2} \backslash(0,0)$ and any symplectic leaf $L$ of $\lambda_{1}\{,\}_{1}+\lambda_{2}\{,\}_{2}$ the intersection $L \cap S$ is Lagrangian in $L$.

Proposition 11.7. Given a micro-Kronecker bihamiltonian structure on $M$ with a base $\mathcal{B}$ of the action foliation, and a cross-section $S$, there is a local isomorphism of bihamiltonian structures $M$ and $\Phi(\mathcal{B})$ which sends $S$ to 0 -section of $\Phi(\mathcal{B})$.

Proof. Given a locally-affine structure $L$ and a point $l \in L$, the exponential mapping of the connection on $\mathcal{T} L$ gives a canonical identification of a neighborhood of $l$ in $L$ and a neighborhood of 0 in $\mathcal{T}_{l} L$. Similarly, given a bundle $\mathcal{L} \rightarrow B$ with fibers having a locally-affine structure, and a section $s$ of this bundle, one obtains a canonical

\footnotetext{
${ }^{33}$ Recall that the space of fixed point of an involution $i: M \rightarrow M$ on a manifold $M$ is a submanifold Fix $(i) \subset M$, and for $f \in \operatorname{Fix}(i)$ the tangent space to $\operatorname{Fix}(i)$ at $f$ coincides with the invariant subspace of the differential $\left.i_{*}\right|_{f}$ of $i$. Indeed, consider an $i$-invariant Riemannian metric on $M$, then the exponential mapping of this mapping commutes with $i$.
} 
identification of a neighborhood of $\operatorname{Im} s \subset \mathcal{L}$ with a neighborhood of 0 -section in an appropriate vector bundle $\mathcal{E} \rightarrow B$. Obviously, fibers of $\mathcal{E}$ are vertical tangent space of $\mathcal{L}$ at points of $\operatorname{Im} s$. In conditions of the proposition $\mathcal{E}=\Phi(B)$.

In particular, any submanifold $S \subset M$ which is transversal to leaves of $\mathcal{F}$, and such that $\operatorname{dim} S=\operatorname{codim} \mathcal{F}$, gives a natural identification $\gamma$ of a neighborhood of $S$ with a neighborhood of 0 -section of $\Phi(\mathcal{B})$ (one can identify $S$ with the local base $\mathcal{B}$ ). We need to show that if $S$ is a cross-section, then this identification is compatible with bihamiltonian structures on $M$ and on $\Phi\left(\mathcal{B}_{U}\right)$.

It is enough to show that $\gamma$ is compatible with $\{,\}_{1}$ (the same argument will be applicable to $\left.\{,\}_{2}\right)$. Consider a symplectic leaf $\widetilde{L} \subset M$ for $\{,\}_{1}$. It is a preimage of a submanifold $L \subset \mathcal{B}_{M}$. The restriction foliation $\left.\mathcal{F}\right|_{\tilde{L}}$ is well-defined and is a Lagrangian foliation. Moreover, $L \cap S$ is Lagrangian. Consider the identification of a neighborhood of $\widetilde{L} \cap S$ in $\widetilde{L}$ with a neighborhood of 0 -section of $\mathcal{T}^{*} L$ (see Proposition 8.3). Since $\widetilde{L} \cap S$ is Lagrangian w.r.t. $\{,\}_{1}$, this identification is compatible with the Poisson structure $\{,\}_{1}$. Composing this identification with $\gamma$, one obtains an identification $\widetilde{\gamma}$ of $\mathcal{T}^{*} L$ with a submanifold of $\Phi\left(\mathcal{B}_{M}\right)$.

Obviously, $\widetilde{\gamma}$ sends a fiber of $\mathcal{T}^{*} L$ to a fiber of $\Phi\left(\mathcal{B}_{M}\right)$, and 0 -section of $\mathcal{T}^{*} L$ into a 0 section of $\Phi\left(\mathcal{B}_{M}\right)$. Due to Remark 9.4, $\widetilde{\gamma}$ is compatible with affine structures on fibers of $\mathcal{T}^{*} L$ and of $\Phi\left(\mathcal{B}_{M}\right)$, and since it sends 0 to 0 , it is a linear mapping of vector bundles $\left.\mathcal{T}^{*} L \stackrel{\widetilde{\gamma}}{\rightarrow} \Phi\right|_{L}$. However, given $\left(\lambda_{1}, \lambda_{2}\right) \in \mathbb{K}^{2} \backslash(0,0),\left.\Phi\right|_{L}$ is canonically isomorphic to $\mathcal{T}^{*} \mathcal{F}_{\lambda_{1}: \lambda_{2}}$ (as in Section 4 ), and by Proposition $9.1 \mathrm{~L}$ is a leaf of the foliation $\mathcal{F}_{1: 0}$. Thus there is a canonical isomorphism $i:\left.\mathcal{T}^{*} L \rightarrow \Phi\right|_{L}$ which is compatible with the Poisson structure $\{,\}_{1}$ on $\Phi$.

It is enough to show that $\widetilde{\gamma}=i$. But this is a direct corollary of the proof of Proposition 9.3.

Proposition 11.8. Consider a micro-Kronecker bihamiltonian structure on $M$. If $\iota$ is an anti-involution of $M$ of defect 0 , then any connected component of Fix $(\iota)$ with index 0 is a cross-section.

Proof. This proposition follows immediately from the following

Lemma 11.9. Consider a symplectic manifold $M$ with a Lagrangian foliation $\mathcal{F}$ and an anti-involution $\iota: M \rightarrow M$ which sends each leaf of $\mathcal{F}$ into itself. Then fixed points of $\iota$ form a Lagrangian submanifold of $M$ which is transversal to leaves of $\mathcal{F}$.

Proof. It is enough to restrict our attention to a neighborhood of Fix $(\iota)$, thus one may assume that $M$ is an open subset of $\mathcal{T}^{*} B$, and $\mathcal{F}$ is the foliation on fibers of projection $M \rightarrow B$. Since the locally-affine structure on fibers of a Lagrangian foliation does not change if one multiplies the Poisson bracket by a number, the restriction of $\iota$ on any leaf of $\mathcal{F}$ induces an affine transformation of this leaf (which is an open subset of $\mathcal{T}_{b} B$ for an appropriate $b \in B$ ).

Recall that the set of fixed points of an involution $\iota$ of $M$ forms a manifold $F \subset M$, and if $f \in F$, then $\mathcal{T}_{f} F \subset \mathcal{T}_{f} M$ coincides with the invariant subspace of $\left.(d \iota)\right|_{\mathcal{T}_{f} M}$. 
Lemma 11.10. Consider a symplectic vector space $V$, and a Lagrangian subspace $l \subset V$. Consider a linear involution $i: V \rightarrow V$ such that $\left[i v_{1}, i v_{2}\right]=-\left[v_{1}, v_{2}\right]$ for any $v_{1}, v_{2} \in V$. Suppose that $i l=l$, and that $i$ induces an identity mapping of $V / l$ into itself. Then $\left.i\right|_{l}$ is multiplication by -1 , and $l$ is a complement to the invariant subspace of $i$ in $V$, which is a Lagrangian subspace of $V$.

Proof. Let $V_{1}$ is the vector subspace of fixed points of $i, V_{-1}$ be the eigenspace of $i$ with eigenvalue -1 . Obviously $V=V_{1} \oplus V_{-1}$, and due to the conditions on $i$ both $V_{1}$ and $V_{-1}$ are isotropic. Thus both $V_{1}$ and $V_{-1}$ are Lagrangian. Similarly, $l=l_{1} \oplus l_{-1}$, $l_{1} \subset V_{1}, l_{-1} \subset V_{-1}$. Let $l_{\lambda}^{\prime}$ be any complementary subspace to $l_{\lambda}$ in $V_{\lambda}, \lambda \in\{1,-1\}$. Since the action of $i$ in $V / l$ is isomorphic to the action of $i$ in $l_{1}^{\prime} \oplus l_{-1}^{\prime}$, we see that $l_{-1}^{\prime}=0$, thus $l \supset V_{-1}$. Since $l$ is Lagrangian, $l=V_{-1}$, which finishes the proof.

Applying this lemma to $V=\mathcal{T}_{f} M, l=\mathcal{T}_{f} L$ (here $f \in M$ is such that if $=f, L$ is the leaf of $\mathcal{F}$ through $f$ ) finishes the proof of Lemma 11.9.

This finishes the proof of Proposition 11.8.

\section{REAL AND COMPLEX BIHAMILTONiAN STRUCTURES}

Consider a real-analytic bihamiltonian structure $\{,\}_{1,2}$ on $M$. One can construct a small complex-analytic neighborhood $M_{\mathbb{C}} \supset M$ of the manifold $M$ such that the brackets $\{,\}_{1,2}$ can be analytically continued on $M_{\mathbb{C}}$. If the bihamiltonian structure on $M$ is micro-Kronecker, one may assume the same about $M_{\mathbb{C}}$ (possibly, after decreasing $\left.M_{\mathbb{C}}\right)$.

Proposition 12.1. Let $m \in M \subset M_{\mathbb{C}}$, suppose that $U \subset M_{\mathbb{C}}, m \in U$, allows an anti-involution $\iota: U \rightarrow U$ with $m=\iota m$ and defect 0 near $m$. Then there is a neighborhood $U_{1}$ of $m$ in $M$ which allows an anti-involution $\iota^{\prime}: U^{\prime} \rightarrow U^{\prime}$ with $m=\iota^{\prime} m$ and defect 0 near $m$.

Proof. Recall that

Definition 12.2. An antiholomorphic mapping $C: N \rightarrow N^{\prime}$ of complex analytic manifolds $N$ and $N^{\prime}$ is such a mapping of sets that $\overline{C^{*} \varphi}$ is a holomorphic function on $N$ if $\varphi$ is a holomorphic function on $N^{\prime}$.

Clearly, if $C$ is antiholomorphic, and $Z \subset N^{\prime}$ is a complex-analytic subvariety, then $C^{-1} Z$ is a complex analytic subvariety. Indeed, if $\varphi=0$ is an equation of $Z$, then $\overline{C^{*} \varphi}=0$ is an equation of $C^{-1} Z$. Similarly, one can transfer a Kronecker structure and a bihamiltonian structure via an antiholomorphic bijection. If $N_{\mathbb{C}}$ is a complexification of a real-analytic manifold $N$, then $N_{\mathbb{C}}$ is equipped with an antiholomorphic involution $C$ such that $\operatorname{Fix}(C)=N$. If $N$ has a Kronecker or bihamiltonian structure, so has $N_{\mathbb{C}}$, and the structures on $N_{\mathbb{C}}$ are invariant w.r.t. $C$.

Consider the antiholomorphic involution $C$ for $M_{\mathbb{C}}, \operatorname{Fix}(C)=M$. Consider a cross-section $\sigma$ of the projection $\pi_{\mathbb{C}}$ which passes through $m$. Then $C \sigma$ is also a crosssection which passes through $m$. Since fibers of $\mathcal{F}$ have an locally-affine structure, the 
section $\frac{\sigma+C \sigma}{2}$ is well defined on an appropriate neighborhood of $\pi m \in \mathcal{B}$. Moreover, this section is a cross-section. Indeed, this immediately follows

Lemma 12.3. Consider a Lagrangian foliation $\mathcal{F}$ on a symplectic manifold $M$, and submanifolds $S_{1}, S_{2}, S_{3}$ of dimension $\frac{\operatorname{dim} M}{2}$ which are transversal to $\mathcal{F}$. Suppose that for any leaf $L$ of $\mathcal{F}$ the intersection $S_{i} \cap L, i=1,2,3$, consists of one point $p_{i}$, and $p_{2}$ is the midpoint of the segment $p_{1} p_{3}$ in the locally affine structure on L. If $L_{1}$ and $L_{3}$ are Lagrangian, so is $L_{2}$.

Proof. Indeed, we may assume that $M=\mathcal{T}^{*} N$, leaves of $\mathcal{F}$ are fibers of $\pi: M \rightarrow N$, $S_{1}$ is 0 -section of $\mathcal{T}^{*} N, S_{2,3}$ are graphs of sections $\varepsilon_{2,3}$ of $\mathcal{T}^{*} N=\Omega^{1} N$, and $\varepsilon_{3}=2 \varepsilon_{2}$.

Lemma 12.4. Consider a section $\varepsilon$ of $\mathcal{T}^{*} B$. The graph of $\varepsilon$ (which is a submanifold of $\mathcal{T}^{*} B$ ) is Lagrangian iff $d \varepsilon=0 \in \Omega^{2} B$.

Application of this obvious lemma finishes the proof of Lemma 12.3.

Now the section $\frac{\sigma+C \sigma}{2}$ of $\pi$ is $C$-invariant, thus it is a complexification of the section $\sigma_{\mathbb{R}}$ for $M$. Since the complexification of $\sigma_{\mathbb{R}}$ is a cross-section, so is $\sigma_{\mathbb{R}}$. This finishes the proof of the proposition.

\section{ENDOMORPHISMS OF BIHAMILTONIAN STRUCTURES AND THE PRINCIPAL THEOREM}

The following statement is widely known:

Proposition 13.1. Suppose that $\left(\lambda_{1}, \lambda_{2}\right)$ and $\left(\lambda_{1}^{\prime}, \lambda_{2}^{\prime}\right)$ are two non-proportional vectors in $\mathbb{K}^{2}$. Consider a bihamiltonian structure $\{,\}_{1,2}$ on a manifold $M$. Suppose that a function $F$ on $M$ is a Casimir function for the bracket $\lambda_{1}\{,\}_{1}+\lambda_{2}\{,\}_{2}$. Let $\chi \in \operatorname{Vect} M$ be the Hamiltonian vector field ${ }^{34}$ of $F$ w.r.t. the bracket $\lambda_{1}^{\prime}\{,\}_{1}+\lambda_{2}^{\prime}\{,\}_{2}$. Consider the flow $\alpha_{t}$ of $\chi$ in time $t \in \mathbb{K}$, as a mapping $\alpha_{t}: U_{1} \rightarrow U_{2}$; here $U_{1,2}$ are open subsets of $M$. Consider $U_{1,2}$ with restriction bihamiltonian structures. Then $\alpha_{t}$ is an isomorphism of bihamiltonian structures.

Proof. Indeed, the Hamiltonian vector field of any function w.r.t. a Poisson bracket $\{$,$\} preserves \{$,$\} . Thus the bracket \lambda_{1}^{\prime}\{,\}_{1}+\lambda_{2}^{\prime}\{,\}_{2}$ is preserved by $\chi$, thus by $\alpha_{t}$. On the other hand, due to the condition on $F, \chi$ is proportional to the Hamiltonian flow of $F$ w.r.t. any bracket $\lambda_{1}^{\prime \prime}\{,\}_{1}+\lambda_{2}^{\prime \prime}\{,\}_{2}$ (as far as $\lambda_{1}^{\prime \prime}: \lambda_{2}^{\prime \prime} \neq \lambda_{1}: \lambda_{2}$ ), thus $\chi$ preserves the Poisson structure $\lambda_{1}^{\prime \prime}\{,\}_{1}+\lambda_{2}^{\prime \prime}\{,\}_{2}$ as well. By linearity, it preserves $\lambda_{1}\{,\}_{1}+\lambda_{2}\{,\}_{2}$ too.

Definition 13.2. For $m \in M$ call $v \in \mathcal{T}_{m} M$ a biflow vector if on a small open subset $U \subset M, U \supset m$, the vector $v$ can be represented as a value of $\chi$ at $m$; here $\chi$ is a vector field from Proposition 13.1.

\footnotetext{
${ }^{34}$ I.e., $\chi=\mathrm{H}(d F)$; here $\mathrm{H}$ is the Hamiltonian mapping $\mathcal{T}^{*}(M) \rightarrow \mathcal{T}(M)$.
} 
Theorem 13.3. Consider a micro-Kronecker bihamiltonian structure on a manifold $M$. Let $m \in M$, let $L$ be the leaf of action foliation on $M$ which passes through $m$. If $m^{\prime} \in L$, then there are neighborhoods $U, U^{\prime}$ of $m$ and $m^{\prime}$ and a diffeomorphism $\alpha: U \rightarrow U^{\prime}$ which

1. sends the restriction bihamiltonian structure on $U$ to the restriction bihamiltonian structure on $U^{\prime}$;

2. for $n \in U_{1}$ the point $\alpha(n)$ is on the same leaf of action foliation as $n$.

Proof. Due to Proposition 13.1 it is enough to show that the span of biflow vectors in $\mathcal{T}_{m} M$ coincides with $\mathcal{T}_{m} L$. Decrease $M$ so that the action foliation becomes a fibration $\pi: M \rightarrow \mathcal{B}$ with a base $\mathcal{B}$. Let $b=\pi(m)$.

Lemma 13.4. Consider the pencil $\widetilde{\mathcal{P}}_{1,2}: \mathcal{T}_{b}^{*} \mathcal{B} \rightarrow \mathcal{T}_{m} L$ from the proof of Proposition 9.3. A vector $v \in \mathcal{T}_{m} M$ is a biflow vector iff $v$ can be written as $\left(\lambda_{1}^{\prime} \widetilde{\mathcal{P}}_{1}+\lambda_{2}^{\prime} \widetilde{\mathcal{P}}_{2}\right) \alpha$ with $\left(\lambda_{1} \widetilde{\mathcal{P}}_{1}+\lambda_{2} \widetilde{\mathcal{P}}_{2}\right) \alpha=0, \lambda_{1}, \lambda_{2}, \lambda_{1}^{\prime}, \lambda_{2}^{\prime} \in \mathbb{K},\left(\lambda_{1}, \lambda_{2}\right) \neq(0,0), \alpha \in \mathcal{T}_{b}^{*} \mathcal{B}$.

Proof. If $f$ is a function on $\mathcal{B}$, and $\chi$ is the Hamiltonian flow of $f \circ \pi$ w.r.t. the Poisson bracket $\lambda_{1}^{\prime}\{,\}_{1}+\lambda_{2}^{\prime}\{,\}_{2}$, then the value of $\chi$ at $m \in M$ coincides with $\left(\lambda_{1}^{\prime} \widetilde{\mathcal{P}}_{1}+\lambda_{2}^{\prime} \widetilde{\mathcal{P}}_{2}\right)\left(\left.d f\right|_{b}\right)$. Since locally any Casimir function for $\lambda_{1}\{,\}_{1}+\lambda_{2}\{,\}_{2}$ can be written as $F=f \circ \pi$ for an appropriate $f$ such that $\left(\lambda_{1} \widetilde{\mathcal{P}}_{1}+\lambda_{2} \widetilde{\mathcal{P}}_{2}\right)\left(\left.d f\right|_{b}\right)=0$, this implies the "only if" part of the lemma.

On the other hand, if $\left(\lambda_{1} \widetilde{\mathcal{P}}_{1}+\lambda_{2} \widetilde{\mathcal{P}}_{2}\right) \alpha=0$, then $\alpha$ is normal to the leaf of the integrating foliation $\mathcal{F}_{\lambda_{1}: \lambda_{2}}$ on $\mathcal{B}$. Decreasing $\mathcal{B}$, one can find a function $f$ on $\mathcal{B}$ such that $f$ is constant on leaves of $\mathcal{F}_{\lambda_{1}: \lambda_{2}}$, and $\left.d f\right|_{b}=\alpha$. This implies the "if" part of the lemma.

Lemma 13.5. Consider a Kronecker relation in a vector space $V$, and the associated pencil $\mathcal{P}_{1,2}: V \rightarrow V^{\prime}$. Let $\widetilde{V}^{\prime}$ be the span of vectors $v^{\prime} \in V^{\prime}$ of the form $\left(\lambda_{1}^{\prime} \mathcal{P}_{1}+\lambda_{2}^{\prime} \mathcal{P}_{2}\right) v$ with $v \in V$ and $\left(\lambda_{1} \mathcal{P}_{1}+\lambda_{2} \mathcal{P}_{2}\right) v=0, \lambda_{1}, \lambda_{2}, \lambda_{1}^{\prime}, \lambda_{2}^{\prime} \in \mathbb{K},\left(\lambda_{1}, \lambda_{2}\right) \neq(0,0)$. Then $\widetilde{V}^{\prime}=V^{\prime}$

Proof. We may assume $\lambda_{1}^{\prime}=1, \lambda_{2}^{\prime}=0$. Since $\mathcal{P}_{1} V=V^{\prime}$, it is enough to show that vectors $v \in V$ such that $\left(\lambda_{1} \mathcal{P}_{1}+\lambda_{2} \mathcal{P}_{2}\right) v=0$ for an appropriate $\lambda_{1}, \lambda_{2} \in \mathbb{K}$ span $V$. But this is a corollary of Lemma 6.3.

Lemma 13.6. In conditions of Proposition 13.1 assume that the bihamiltonian structure on $M$ is micro-Kronecker, and $\mathcal{F}$ is the action foliation. Then for any $m \in U_{1}$ points $m$ and $\alpha_{t} m$ are on the same leaf of $\mathcal{F}$.

Proof. This follows from the fact that biflow vectors are tangent to leaves of action foliation.

This finishes the proof of Theorem 13.3. 
Theorem 13.7. Consider a micro-Kronecker bihamiltonian structure on a manifold $M$ and an anti-involution $\alpha$ of $M$. Let $Z$ be the submanifold formed by the fixed points of $\alpha$ of defect 0 . Let $U$ be the union of leaves of the action foliation of $M$ which intersect $Z$. Then

1. The subset $U \subset M$ is open;

2. If $m \in U$, then Conjecture 10.1 holds for $m$ and $M$.

Proof. This is an immediate corollary of Theorems 11.5 and 13.3.

Proposition 11.2 and Theorem 13.7 show that in fact Conjectures 10.1 and 11.4 are equivalent to each other. However, the rôles of these conjecture are very dissimilar. As we will show in Section 14, for some particular bihamiltonian structures of mathematical physics Conjecture 11.4 is easy to verify by an explicit construction (see Theorem 14.14), thus for these structures Conjecture 10.1 follows from this construction.

On the other hand, as it was shown in [13], in the case an arbitrary bihamiltonian structure of rank 1 it is possible to prove Conjecture 10.1 using some "hard" cohomological statements. One can expect that a similar approach may succeed in the case of higher rank as well. But currently it is not clear how one could prove Conjecture 11.4 without a reference to Conjecture 10.1 (or the calculation of some cohomology which will immediately prove Conjecture 10.1).

This suggests that in some particular cases it is easier to directly deduce the statement of Conjecture 11.4, but in general case Conjecture 10.1 should be easier to tackle.

\section{Method of argument translation}

Consider a Lie algebra $\mathfrak{g}$ and a 2-cocycle $c_{2}$ of $\mathfrak{g}$. As in Example 0.10, such a pair induces a bihamiltonian structure $\{,\}_{1,2}$ on $\mathfrak{g}^{*}$. Moreover, if $c_{2}$ is a coboundary of a 1-chain $c_{1}$, one may consider $c_{1}$ as an element of $\mathfrak{g}^{*}$. Obviously, the Poisson structure $\{,\}_{1}$ is a Lie derivative of the Poisson structure $\{,\}_{2}$ in the direction of a parallel translation of $\mathfrak{g}^{*}$ in the direction of $c_{1} \in \mathfrak{g}^{*}$, and $\{,\}_{1}$ is translation-invariant. Thus $\{,\}_{2}+\lambda\{,\}_{1}$ is a parallel translation of $\{,\}_{2}$ by $\lambda c_{1}$. Due to this observation consideration of the pair \{\}$_{1,2}$ when $c_{2}$ is a coboundary is often called the method of argument translation.

Moreover, if $\mathfrak{g}$ is semisimple, then $c_{2}$ is automatically a coboundary.

Definition 14.1. Given $c_{1} \in \mathfrak{g}^{*}$, let $\{,\}_{1,2}$ be the bihamiltonian structure on $\mathfrak{g}^{*}$ constructed based on 2-coboundary $d c_{1}$. Call this structure the associated to $c_{1} \in \mathfrak{g}^{*}$ structure.

Definition 14.2. Given a Lie algebra $\mathfrak{g}$ over $\mathbb{K}$, let $\mathfrak{g}_{\mathbb{C}} \stackrel{\text { def }}{=} \mathfrak{g}$ if $\mathbb{K}=\mathbb{C}$, and $\mathfrak{g}_{\mathbb{C}} \stackrel{\text { def }}{=} \mathfrak{g} \otimes \mathbb{C}$ if $\mathbb{K}=\mathbb{R}$. 
Lemma 14.3. Consider the Poisson structure $\{,\}_{2}$ on $\mathfrak{g}^{*}$ and $\alpha \in \mathfrak{g}^{*}$. Let $L$ be the symplectic leaf of $\{,\}_{2}$ through $\alpha$. Then $\mathcal{N}_{\alpha}^{*} L=\operatorname{Stab}_{a^{*}} \alpha$.

The proof of this lemma is a direct calculation.

Recall description of the geometry of the set of regular elements in a dual space to a Lie algebra.

Definition 14.4. The rank $\mathrm{rk}(\mathfrak{g})$ of a Lie algebra $\mathfrak{g}$ is $\min _{\alpha \in \mathfrak{g}_{\mathbb{C}}^{*}} \operatorname{dim} \operatorname{Stab}_{\mathrm{Ad}^{*}} \alpha$. An element $\alpha \in \mathfrak{g}^{*}$ is regular if $\operatorname{dim} \operatorname{Stab}_{\mathrm{Ad}^{*}} \alpha=\mathrm{rk}(\mathfrak{g})$, and irregular otherwise.

Obviously, regular elements form a (Zariski) open and dense subset of $\mathfrak{g}^{*}$.

Definition 14.5. Let $\alpha \in \mathfrak{g}^{*}, \beta \in \mathfrak{g}_{\mathbb{C}}^{*}$. Call $\beta$ compatible with $\alpha$ if $\beta+\lambda \alpha$ is regular for any $\lambda \in \mathbb{C}$. Call a regular element $\alpha \in \mathfrak{g}^{*}$ strongly regular if there exists a compatible with $\alpha$ element of $\mathfrak{g}_{\mathbb{C}}^{*}$.

Definition 14.6. Call $\mathfrak{g} 2$-regular, if the algebraic subvariety $\mathcal{I} \subset \mathfrak{g}_{\mathbb{C}}^{*}$ of irregular elements has codimension 2 or more.

Proposition 14.7. Suppose that there exists a strongly regular element $\alpha$ in $\mathfrak{g}_{\mathbb{C}}^{*}$. Then $\mathfrak{g}$ is 2-regular.

Suppose that $\mathfrak{g}$ is 2-regular, and $\alpha$ is a regular element of $\mathfrak{g}^{*}$. Then $\alpha$ is strongly regular, and the set of elements of $\mathfrak{g}^{*}$ which are compatible with $\alpha$ is non-empty and Zariski open.

Proof. Let $\mathcal{I} \subset \mathfrak{g}_{\mathbb{C}}^{*}$ be the subvariety of irregular elements. Let $\pi$ be the projection of $\mathfrak{g}_{\mathbb{C}}^{*}$ to $\mathfrak{g}_{\mathbb{C}}^{*} / \mathbb{K} \alpha$. The existence of an $\alpha$-compatible element is equivalent to $\pi \mathcal{I} \neq \pi \mathfrak{g}_{\mathbb{C}}^{*}$.

If $\beta$ is regular, then any non-zero scalar multiple of $\beta$ is also regular. Thus one can consider a closed subvariety $\mathbb{P} \mathcal{I}$ of irregular elements in the projectivization $\mathbb{P} \mathfrak{g}^{*}$ of $\mathfrak{g}^{*}$. Given a strongly regular element $\alpha$ and a compatible element $\beta$, one obtains a line $l=\mathbb{P}\langle\alpha, \beta\rangle$ in $\mathbb{P g}^{*}$, and $l \cap \mathbb{P} \mathcal{I}=\varnothing$. Clearly, any nearby line $l^{\prime}$ will also not intersect $\mathbb{P} \mathcal{I}$. Thus the set of elements $\beta$ which are compatible with $\alpha$ is Zariski open. Thus the intersection of this set with $\mathfrak{g}^{*} \subset \mathfrak{g}_{\mathbb{C}}^{*}$ is non-empty.

Since $l \cap \mathbb{P} \mathcal{I}=\varnothing, \mathbb{P} \mathcal{I}$ has codimension 2 or more, thus the same is true for $\mathcal{I}$. On the other hand, if $\mathcal{I}$ has codimension 2 or more, then the projection of $\mathcal{I}$ to $\mathfrak{g}^{*} / \mathbb{K} \alpha$ is not surjective; here $\alpha \in \mathfrak{g}^{*}$ is arbitrary. This implies that any regular element of $\mathfrak{g}^{*}$ is strongly regular.

Proposition 14.8. Suppose that $c_{1} \in \mathfrak{g}^{*}$ is strongly regular. Then there is a dense open subset $U \subset \mathfrak{g}^{*}$ such that the restriction on $U$ of the pair $\{,\}_{1,2}$ associated to $c_{1}$ is micro-Kronecker of rank $\mathrm{rk}(\mathfrak{g})$.

Proof. By Proposition 14.7, the set $U$ of compatible with $c_{1}$ elements of $\mathfrak{g}_{\mathbb{C}}^{*}$ is Zariski open (thus dense). Show that $U$ (or $U \cap \mathfrak{g}$ in the case $\mathbb{K}=\mathbb{R}$ ) satisfies the conditions of the proposition. One may assume that $\mathbb{K}=\mathbb{C}$.

We need to show that for $\beta \in U$ the symplectic leaf through $\beta$ of $\lambda_{1}\{,\}_{1}+\lambda_{2}\{,\}_{2}$, $\left(\lambda_{1}, \lambda_{2}\right) \neq(0,0)$, has codimension $\mathrm{rk}(\mathfrak{g})$. For $\lambda_{2}=0$ the normal space to this leaf 
coincides with $\mathrm{Stab}_{\mathrm{Ad}^{*}} c_{1}$, thus regularity of $c_{1}$ implies the statement. Thus we may assume $\lambda_{2}=1$. The Poisson structure $\lambda\{,\}_{1}+\{,\}_{2}$ is a $\lambda c_{1}$-translation of $\{,\}_{2}$. If $\beta \in U$, then $\beta_{1}=\lambda c_{1}+\beta$ is regular.

Thus it is enough consider $\lambda=0$. But the normal space to the leaf of symplectic foliation through $\beta_{1}$ is $\operatorname{Stab}_{\mathrm{ad}^{*}} \beta_{1}$, which finishes the proof.

Proposition 14.9. If $\mathfrak{g}$ is reductive, then any regular element $\alpha \in \mathfrak{g}^{*}$ is strongly regular.

Proof. Indeed [1], irregular elements of a semisimple Lie algebra form a Zariski closed subvariety of codimension 3. This implies that the same statement for reductive algebras, thus the proposition.

The arguments above are not new, see [4, 27].

The last proposition cannot be inverted:

Example 14.10. (Proposed by V. Serganova) For any vector space $V$ there is a canonical symmetric pairing on $V \oplus V^{*}$. For any Lie algebra $\mathfrak{g}$ this pairing on $\mathfrak{g} \oplus \mathfrak{g}^{*}$ is an invariant pairing on the Lie algebra $\mathfrak{G}=\mathfrak{g} \ltimes \mathrm{ad}_{\mathfrak{g}}^{*}$; here $\mathrm{ad}_{\mathfrak{g}}^{*}$ is the adjoint representation of $\mathfrak{g}$ with trivial structure of Lie algebra. This gives an isomorphism $\mathfrak{G} \simeq \mathfrak{G}^{*}$ of $\mathfrak{G}$-modules.

This allows one to replace the study of dimensions of stabilizers of elements of $\mathfrak{G}^{*}$ by the study of dimensions of stabilizers of elements of $\mathfrak{G}$. If $\mathfrak{g}=\mathfrak{s l}_{2}$, it is easy to show that the set of irregular elements coincides with the radical of $\mathfrak{G}$, which has codimension 3. Later, in Example 14.31, we will see that this statement on codimension holds for other reductive algebras too.

Now show how one can refine the description of bihamiltonian structure on $\mathfrak{g}$ by applying the general machinery of this paper.

Definition 14.11. A linear mapping $\iota: \mathfrak{g} \rightarrow \mathfrak{g}$ is an antiinvolution of $\mathfrak{g}$ if $\iota$ is an involution of a vector space, and $[\iota X, \iota Y]=-\iota[X, Y]$ for any $X, Y \in \mathfrak{g}$. An antiinvolution $\iota$ is admissible, if

1. The irregular elements in the vector subspace Fix $\left(\iota^{*}\right) \subset \mathfrak{g}^{*}$ of fixed points of $\iota$ in $\mathfrak{g}^{*}$ form a subvariety of Fix $\left(\iota^{*}\right)$ of codimension 2 or more.

2. The subset $\mathcal{U}(\iota) \subset \operatorname{Fix}\left(\iota^{*}\right)$ consisting of points $\alpha \in \operatorname{Fix}\left(\iota^{*}\right)$ such that the $\mathrm{Ad}^{*}$ orbit of $\alpha$ is transversal to Fix $\left(\iota^{*}\right)$ is not empty;

Call $\alpha \in \mathfrak{g}^{*}$ admissible, if $\alpha$ is regular, and there is an admissible anti-involution $\iota$ such that $\iota \alpha=\alpha$.

Remark 14.12. Obviously, admissible elements exists only in 2-regular Lie algebras, and are strongly regular. Clearly, for an admissible anti-involution $\iota$ the set $\mathcal{U}(\iota)$ is Zariski open in Fix $\left(\iota^{*}\right)$. Moreover, if $\alpha$ is admissible, and $\iota$ is the corresponding admissible anti-involution, then elements $\beta \in \operatorname{Fix}\left(\iota^{*}\right)$ which are compatible with $\alpha$ form a non-empty Zariski open subset of Fix $\left(\iota^{*}\right)$. 
Theorem 14.13. Suppose that $c_{1} \in \mathfrak{g}^{*}$ is admissible. Consider the bihamiltonian structure $\{,\}_{1,2}$ associated to $c_{1}$. Then there is an open subset $M \subset \mathfrak{g}^{*}$ such that for any $m \in M$ Conjecture 10.1 holds.

Proof. Consider an admissible anti-involution $\iota$ such that $\iota^{*} c_{1}=c_{1}$. Let $U$ be the Zariski open subset of $\mathfrak{g}_{\mathbb{C}}^{*}$ where $\{,\}_{1,2}$ is micro-Kronecker. Since $\iota$ is an anti-involution of $\mathfrak{g}, \iota^{*}$ is an anti-involution of the Poisson structure $\{,\}_{2}$ on $\mathfrak{g}^{*}$. Since $\iota^{*}$ preserves $c_{1}$, and $\{,\}_{1}$ is the derivative of $\{,\}_{2}$ w.r.t. translations in the direction of $c_{1}, \iota^{*}$ is an anti-involution of $\{,\}_{1}$ as well. Thus $U$ is $\iota^{*}$-invariant, and $\left.\iota^{*}\right|_{U}$ is an anti-involution of a micro-Kronecker bihamiltonian structure.

Note that the same arguments as in the proof of Proposition 14.7 show that $U \cap$ Fix $\left(\iota^{*}\right) \neq \varnothing$. Let $\widetilde{U}=U \cap \mathcal{U}\left(\iota^{*}\right)$. This is a non-empty Zariski open subset of Fix $\left(\iota^{*}\right)$. Let $\beta \in \widetilde{U}$. The principal step is to show that the defect of $\iota^{*}$ at $\beta$ is 0 .

Let $U_{1}$ be a neighborhood of $\beta$ in $U$ such that the action foliation $\mathcal{F}$ of the bihamiltonian structure becomes a fibration $\pi: U_{1} \rightarrow \mathcal{B}$. It is enough to show that for any function $\varphi$ on $\mathcal{B}$ the function $\varphi \circ \pi$ on $U_{1}$ is preserved by $\iota^{*}$. In turn, it is enough to do the same for a large enough collection of functions $\varphi$ on $\mathcal{B}$. Take as such collection functions $\varphi$ which are constant on fibers of the integrating foliation $\mathcal{F}_{\lambda}$ on $\mathcal{B}$, for each one of $\lambda \in \Lambda$ (for a sufficiently large $\Lambda \subset \mathbb{K}^{1}$ ). We may suppose $\Lambda \subset \mathbb{K}$.

Obviously, $\varphi$ is constant on fibers of $\mathcal{F}_{\lambda}$ iff $\varphi \circ \pi$ is constant on fibers of the symplectic foliation $\widetilde{\mathcal{F}}_{\lambda}$ of the Poisson structure $\lambda\{,\}_{1}+\{,\}_{2}$. Thus it is enough to show that $\iota^{*}$ preserves such functions. Since $\lambda\{,\}_{1}+\{,\}_{2}$ is the result of translation of $\{,\}_{2}$ by $\lambda c_{1}$, it is enough to show this for $\lambda=0$ and $\beta+\lambda c_{1}, \lambda \in \Lambda$, taken instead of $\beta$.

However, $\beta^{\prime}=\beta+\lambda c_{1}$ is in $\widetilde{U}$ for $\lambda$ in an open subset of $\mathbb{K}$, thus we can restrict our attention to a given $\beta^{\prime} \in \widetilde{U}$ and $\lambda=0$. Since $\beta^{\prime} \in \mathcal{U}\left(\iota^{*}\right)$, any $\mathrm{Ad}^{*}$-orbit which passes near $\beta^{\prime}$ intersects Fix $\left(\iota^{*}\right)$. Since $\iota^{*}$ sends an $\mathrm{Ad}^{*}$-orbit to an $\mathrm{Ad}^{*}$-orbit, this implies that $\iota^{*}$ preserves any $\mathrm{Ad}^{*}$-orbit which passes near $\beta^{\prime}$. Decrease $U_{1}$ so that $\mathrm{Ad}^{*}$-orbit of any $\gamma \in U_{1}$ intersects Fix $\left(\iota^{*}\right)$. However, symplectic leaves of $\{,\}_{2}$ coincide with $\mathrm{Ad}^{*}$-orbits in $\mathfrak{g}^{*}$. Thus on a neighborhood of $\beta^{\prime}$ any function which is constant on symplectic leaves of $\{,\}_{2}$ is $\iota^{*}$-invariant.

This implies that the defect of $\iota^{*}$ near $\beta$ is indeed 0 , and we are in conditions of Theorem 13.7. This implies the theorem for $M$ being the union of fibers of the action foliation which intersect $\widetilde{U}$.

Theorem 14.14. Suppose that $\mathfrak{g}$ is semisimple, and $c_{1} \in \mathfrak{g}^{*}$ is regular and semisimple. Then there is a dense open subset $U \subset \mathfrak{g}^{*}$ such that the restriction on $U$ of the pair $\{,\}_{1,2}$ associated to $c_{1}$ satisfies Conjecture 10.1.

Proof. Identify $\mathfrak{g}^{*}$ with $\mathfrak{g}$ using the Killing form. Due to Proposition 12.1, it is enough to consider the case $\mathbb{K}=\mathbb{C}$. 
Definition 14.15. Consider a semisimple Lie algebra $\mathfrak{g}$. Define the Cartan antiinvolution $\iota$ by its action on standard generators $e_{i}, f_{i}, h_{i}, i=1, \ldots, r$ :

$$
\iota\left(e_{i}\right)=f_{i}, \iota\left(f_{i}\right)=e_{i}, \iota\left(h_{i}\right)=h_{i} .
$$

Lemma 14.16. The Cartan anti-involution of a semisimple Lie algebra is admissible.

Proof. Identification of $\mathfrak{g}$ with $\mathfrak{g}^{*}$ allows one to consider $\iota$ instead of $\iota$. Any antiinvolution sends an Ad-orbit to an Ad-orbit. Since Fix $(\iota) \supset \mathfrak{h}$, and an orbit of a regular element of $\mathfrak{h}$ is transversal to $\mathfrak{h}, \iota$ satisfies the second condition of Definition 14.11. Thus to prove the lemma it is enough to show that irregular elements in Fix $(\iota)$ form a subvariety of codimension 2 or more. (Note that this statement is not true if one substitutes $\mathfrak{h}$ instead of Fix $(\iota)$ !)

Due to homogeneity of the set of regular elements, a translation to algebraic geometry in a projective space show that it is enough to prove this statement for an arbitrary vector subspace in Fix $(\iota)$ taken instead of Fix ( $\iota)$ (see Proposition 14.7). Recall that [5]:

Lemma 14.17. There are numbers $a_{i} \neq 0, b_{i} \neq 0, c_{i}, i=1, \ldots, r$, such that for the elements $E=\sum a_{i} e_{i}, F=\sum b_{i} f_{i}, H=\sum c_{i} h_{i}$ the vector subspace $V=\langle E, F, H\rangle \subset$ $\mathfrak{g}$ is a Lie subalgebra isomorphic to $\mathfrak{s l}_{2}$. The adjoint action of $V$ on $\mathfrak{g}$ is a direct sum of $r$ odd-dimensional irreducible representations of $\mathfrak{s l}_{2}$.

Since the action of any non-zero element of $\mathfrak{s l}_{2}$ in an odd-dimensional irreducible representation has 1-dimensional null-space, this shows that the stabilizer of any nonzero point of $V$ has dimension $r$. But $\mathrm{rk} \mathfrak{g}=r$, thus all the non-zero elements of $V$ are regular. Moreover, conjugating $V$ with elements of the Lie group $\exp (\mathfrak{h})$ of $\mathfrak{h}$, one may assume that $a_{i}=b_{i}, i=1, \ldots, r$. Thus the subspace $V$ is $\iota$-invariant, let $V_{0}$ be the 2-dimensional invariant subspace $\langle H, E+F\rangle$ of $\left.\iota\right|_{V}$.

Since $V_{0} \subset \operatorname{Fix}(\iota)$ intersects irregular elements on $\{0\}$, which is a subvariety of codimension 2, irregular elements in Fix $(\iota)$ form a submanifold of codimension 2 or more. This finishes the proof of Lemma 14.16.

Lemma 14.16 implies that any regular $c_{1} \in \mathfrak{g}^{*}$ which is on an $\mathrm{Ad}^{*}$-orbit of Fix $\left(\iota^{*}\right)$ is admissible. But any regular semisimple element of $\mathfrak{g}$ is conjugate to an element of $\mathfrak{h}$, it is on an Ad-orbit of an $\iota$-invariant element! Translating from $\mathfrak{g}$ to $\mathfrak{g}^{*}$, any regular semisimple $c_{1} \in \mathfrak{g}^{*}$ is admissible. Hence there is an open subset $U \subset \mathfrak{g}^{*}$ on which Conjecture 10.1 holds.

Let us show that $U$ can be taken Zariski open, thus dense. Let $U_{0}$ be the Zariski open subset where foliation $\mathcal{F}$ makes sense. Recall that $U$ is the union of leaves of $\mathcal{F}$ which intersect Fix $(\iota)$. Show that $U$ contains a Zariski open subset.

There is a Zariski open subset $U_{1}$ of $\mathfrak{g}$ such that $U_{1}$ is Ad-invariant, and Ad-invariant polynomials distinguish ${ }^{35}$ Ad-orbits in $U_{1}$. On the other hand, locally the fibers of the action foliation $\mathcal{F}$ are intersections of a finite number of $\lambda c_{1}$-shifted Ad-orbits,

\footnotetext{
${ }^{35}$ In the same sense as in Remark 0.3.
} 
$\lambda \in \Lambda$, card $(\Lambda)<\infty$. Taking a large enough finite collection $p_{i}, i \in I$, of invariant polynomials on $\mathfrak{g}$, we see that fibers of $\mathcal{F}$ coincide with connected components of level sets of $p_{i, \lambda}, i \in I, \lambda \in \Lambda$; here $p_{i, \lambda}(X) \stackrel{\text { def }}{=} p_{i}\left(X+\lambda c_{1}\right)$. Let $U_{2}=U_{0} \cap \bigcap_{\lambda \in \Lambda}\left(U_{1}-\lambda c_{1}\right)$; here $U_{1}-\lambda c_{1}$ is the parallel translation of $U_{1}$ by $-\lambda c_{1}$. Obviously, $U_{2}$ is Zariski open and is a union of fibers of foliation $\mathcal{F}$.

Let $\Pi$ be the polynomial mapping of $\mathfrak{g}$ to $\mathbb{C}^{N}, N=\operatorname{card}(I) \operatorname{card}(\Lambda)$, with com-

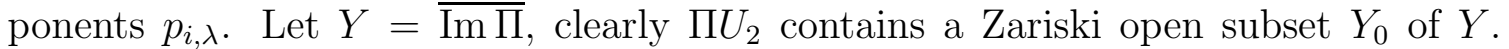
Decreasing $Y_{0}$, one may assume that $\left.\Pi\right|_{\Pi^{-1} Y_{0}}$ is a submersion to $Y_{0}$. Since $\Pi$ is constant on leaves of $\mathcal{F}$, $\Pi$ Fix $(\iota)=\Pi U$. Since $U$ contains an open subset of $\mathfrak{g}$, $\Pi$ Fix $(\iota)$ contains an open subset of $Y$, thus a Zariski open subset of $Y$. Thus one can assume that $Y_{0} \subset \Pi \operatorname{Fix}(\iota)$. Let $U_{3}=\Pi^{-1} Y_{0}, Z=U_{3} \cap \operatorname{Fix}(\iota)$.

We obtain the following mappings: $Z \stackrel{i}{\hookrightarrow} U_{3} \stackrel{\Pi}{\rightarrow} Y_{0}$; here $U_{3}$ is Zariski open in $\mathfrak{g}, Z$ is Zariski closed in $U_{3}, \Pi$ is (a restriction of) a polynomial mapping which is a submersion onto $Y_{0}$, and $\Pi \circ i$ is surjective. Let $U_{4}$ be the union of connected components of fibers of $\Pi$ which intersect $Z$. Since $U_{4} \subset U$, it is enough to show that $U_{4}$ coincides with $U_{3}$ (which is a union of fibers of $\pi$ which intersect $Z$ ).

Since $\Pi$ is a submersion, the number of connected components of fibers of $\Pi$ can only jump up during specialization. This implies that $U_{4}$ is open in $U_{3}$. Decreasing $Y_{0}$, we may assume that the number of connected components of the fiber of $\Pi$ over $z$ does not depend on $z \in Y_{0}$. This implies that $U_{4}$ is closed in $U_{3}$. Since $U_{3}$ is connected (as a Zariski open subset of a vector space), $U_{3}=U_{4}$. This finishes the proof of Theorem 14.14.

Amplification 14.18. In Theorem 14.14 one can take $\mathfrak{g}$ being reductive, and drop the condition of semisimplicity on $c_{1}$.

Proof. As the proof of Theorem 14.14 shows, it is enough to show that

Proposition 14.19. Consider a reductive Lie algebra $\mathfrak{g}$ and its Cartan anti-involution $\iota$. Then any element $X$ of $\mathfrak{g}$ is on Ad-orbit of $\iota$-invariant element.

Proof (V. Serganova) . First of all, the statement for reductive algebras follows momentarily from the case of semisimple Lie algebras. For semisimple elements the statement is obvious (since $\mathfrak{h}$ is $\iota$-invariant). Consider the case when $X \in \mathfrak{g}$ is nilpotent.

One may assume that $X \neq 0$. If $\mathfrak{g}=\mathfrak{s l}_{2}$, then any nilpotent element is $\mathrm{SL}_{2^{-}}$ conjugate to $\left(\begin{array}{cc}1 & i \\ i & -1\end{array}\right)$ which is symmetric, thus $\iota$-invariant. Thus to prove the proposition for the case of nilpotent $X$ is enough to show

Lemma 14.20. For any nilpotent $X \in \mathfrak{g}$ with a reductive Lie algebra $\mathfrak{g}$ there is a subalgebra $\mathfrak{g}_{0} \subset \mathfrak{g}$ such that $\mathfrak{g}_{0} \simeq \mathfrak{s l}_{2}, \iota \mathfrak{g}_{0}=\mathfrak{g}_{0},\left.\iota\right|_{\mathfrak{g}_{0}}$ is the Cartan anti-involution of $\mathfrak{s l}_{2}$, and $X$ is conjugate to $X^{\prime} \in \mathfrak{g}_{0}$. Here $\iota$ is the Cartan anti-involution of $\mathfrak{g}$. 
Proof. It is enough to prove this for a semisimple $\mathfrak{g}$. The classification of nilpotent elements up to conjugation is well-known ([6], or it can be deduced from [5]):

Lemma 14.21. For any nilpotent element $X \in \mathfrak{g}, X \neq 0$, there is a reductive subalgebra $\widetilde{\mathfrak{g}}$ of $\mathfrak{g}$ with $\mathrm{rk}(\widetilde{\mathfrak{g}})=\mathrm{rk}(\mathfrak{g})$ and a Cartan set of generators $\widetilde{e}_{i}, \widetilde{f}_{i}, i=1, \ldots, \widetilde{r}$, $\widetilde{h}_{j}, j=1, \ldots, \operatorname{rk}(\widetilde{\mathfrak{g}})$, of $\widetilde{\mathfrak{g}}$ such that $X=\sum_{i=1}^{\widetilde{r}} \widetilde{e}_{i}$. Here $\widetilde{r}=\operatorname{rk}(\widetilde{\mathfrak{g}})-\operatorname{dim} Z(\widetilde{\mathfrak{g}})$.

The Cartan subalgebra of $\widetilde{\mathfrak{g}}$ is a Cartan subalgebra of $\mathfrak{g}$, thus after conjugation one can assume that $\widetilde{h}_{j}$ generate $\mathfrak{h} \subset \mathfrak{g}$. Then $\iota \widetilde{h}_{j}=\widetilde{h}_{j}$ for any $j=1, \ldots, \operatorname{rk}(\widetilde{\mathfrak{g}})$. Let $a_{i j}$ be coefficients in relations $\left[\widetilde{h}_{j}, \widetilde{e}_{i}\right]=a_{i j} \widetilde{e}_{i},\left[\widetilde{h}_{j}, \widetilde{f}_{i}\right]=-a_{i j} \widetilde{f}_{i}$. Given $a_{i j}$ and $\widetilde{h}_{i}$, these relations determine $\widetilde{e}_{i}, \widetilde{f}_{i}$ uniquely up to proportionality. This implies that $\iota \widetilde{e}_{i}$ is proportional to $\widetilde{f}_{i}$. Thus $\iota \widetilde{\mathfrak{g}}=\widetilde{\mathfrak{g}}$, moreover, after a rescaling $\left.\iota\right|_{\mathfrak{g}}$ may be supposed to be the Cartan anti-involution of $\widetilde{\mathfrak{g}}$.

Substituting $\widetilde{\mathfrak{g}}$ instead of $\mathfrak{g}$, it follows that it is enough to prove the statement of Lemma 14.20 for $X=\sum_{i=1}^{\mathrm{rk}(\mathfrak{g})} e_{i}$. In this case Lemma 14.17 implies that $X$ is $\exp (\mathfrak{h})$-conjugate to the element $E$ of Lemma 14.17. Moreover, doing another exp (h)conjugation one can ensure that the vector space $\langle E, F, H\rangle$ of Lemma 14.17 is $\iota$ invariant.

Now the proposition is proven for semisimple and for nilpotent elements $X \in \mathfrak{g}$. For an arbitrary $X \in \mathfrak{g}$, there is unique representation $X=X_{s s}+X_{\text {nil }}$ as a sum of commuting semisimple and nilpotent elements. Doing conjugation, we may assume $X_{s s} \in \mathfrak{h}$. Let $\mathfrak{g}_{0}=\mathrm{Stab}_{\mathrm{ad}} X_{s s}$. Obviously, $\iota \mathfrak{g}_{0}=\mathfrak{g}_{0}, \mathfrak{g}_{0}$ is a reductive Lie algebra, $\mathfrak{g}_{0} \supset \mathfrak{h}$, and $\left.\iota\right|_{\mathfrak{g}_{0}}$ is the Cartan anti-involution of $\mathfrak{g}_{0}$. Since $X_{\text {nil }} \in \mathfrak{g}_{0}$, we know that $X_{\text {nil }}$ is $G_{0}$-conjugate to an element of Fix $\left(\left.\iota\right|_{\mathfrak{g}_{0}}\right)$; here $G_{0}$ is the Ad-group of $\mathfrak{g}_{0}$. Since $X_{s s}$ is $G_{0}$-invariant and $\iota$-invariant, the conjugation by $g \in G_{0}$ above sends $X_{s s}+X_{\text {nil }}$ to Fix $\left(\left.\iota\right|_{\mathfrak{g}_{0}}\right) \subset \operatorname{Fix}(\iota)$. This finishes the proof of the proposition.

This finishes the proof of the amplification.

Finally, one can apply the accumulated information to prove a generalization of one of conjectures of [11]:

Theorem 14.22. Suppose that $\mathfrak{g}$ is reductive, and $c_{1} \in \mathfrak{g}^{*}$ is regular. Then there is a dense open subset $U \subset \mathfrak{g}^{*}$ such that the restriction on $U$ of the pair $\{,\}_{1,2}$ is flat ${ }^{36}$.

Proof. Due to Theorem 14.14, it is enough to show that on a dense open subset of $\mathfrak{g}^{*}$ the Kronecker web which is a (local) base of the action foliation is flat $^{37}$. This was proven in [28] (in less generality); here we reproduce a more general form of these arguments:

Lemma 14.23. Consider a Lie algebra $\mathfrak{g}, \mathrm{rk} \mathfrak{g}=r$, and $\mathrm{Ad}^{*}$-invariant polynomials $p_{1}, \ldots, p_{r}$ on $\mathfrak{g}^{*}$. Let $U \subset \mathfrak{g}^{*}$ consists of points $\alpha \in \mathfrak{g}^{*}$ such that $\left.d p_{1}\right|_{\alpha}, \ldots,\left.d p_{r}\right|_{\alpha}$ are

\footnotetext{
${ }^{36}$ Flat bihamiltonian structures were introduced in Definition 0.8.

${ }^{37}$ I.e., locally isomorphic to a translation-invariant structure.
} 
linearly independent. Suppose that $\mathfrak{g}$ is 2-regular, $U \neq \varnothing$, $\operatorname{dim} \mathfrak{g}=2 \sum_{i=1}^{r} \operatorname{deg} p_{i}+r$, and that $c_{1} \in \mathfrak{g}^{*}$ is regular. Consider the Kronecker structure on the local base $\mathcal{B}$ of the action foliation $\mathcal{F}$ of the bihamiltonian structure on $\mathfrak{g}^{*}$ associated to $c_{1}$. This Kronecker structure is flat on an open subset.

Proof. Argue as in the end of the proof of Theorem 14.14. On a Zariski open subset $U_{0}$ of $\mathfrak{g}^{*}$ the polynomials $p_{i}, i=1, \ldots, r$, locally distinguish $\mathrm{Ad}^{*}$-orbits (thus symplectic leaves of $\left.\{,\}_{2}\right)$ on $\mathfrak{g}^{*}$.Thus on a Zariski open subset $U_{1}$ of $\mathfrak{g}^{*}$ the polynomials $p_{i, \lambda}$, $i=1, \ldots, r, \lambda \in \mathbb{K}$, locally distinguish leaves of $\mathcal{F}$ (here $\left.p_{i, \lambda}(\alpha) \stackrel{\text { def }}{=} p_{i}\left(\alpha+\lambda c_{1}\right)\right)$. Associate to a given $\alpha \in \mathfrak{g}^{*}$ the coefficients $a_{i j}, i=1, \ldots, r, j=0, \ldots, \operatorname{deg} p_{i}$, of polynomials $p_{i}\left(\alpha+\lambda c_{1}\right)$ in $\lambda$. This is a polynomial mapping $a: \mathfrak{g}^{*} \rightarrow \mathbb{K}^{N}, N=$ $\sum_{i=1}^{r} \operatorname{deg} p_{i}+r$. We conclude that on $U_{1}$ connected components of fibers of $\left.a\right|_{U_{0}}$ coincide with leaves of $\mathcal{F}$.

But the leaves of $\mathcal{F}$ have codimension $\frac{\operatorname{dim} \mathfrak{g}+r}{2}$, thus in the conditions of the lemma the mapping $\left.a\right|_{U_{0}}$ is a submersion, and leaves of $\mathcal{F}$ are connected components of fibers of $a$. Thus on $U_{1}$ the manifold $a\left(U_{1}\right)$ may be considered as a base $\mathcal{B}$ of the action foliation. Describe the structure of Kronecker web on $a\left(U_{1}\right)$.

Fix $\lambda \in \mathbb{K}$. The symplectic leaves of $\lambda\{,\}_{1}+\{,\}_{2}$ are $-\lambda c_{1}$-translations of $\mathrm{Ad}^{*}-$ orbits, thus on $U_{1}$ they coincide with level sets of $p_{i, \lambda}$. Thus the projections of these leaves to $\mathcal{B}$ may be described by equations $\sum_{j} a_{i j} \lambda^{j}=C_{i}, i=1, \ldots, r$; here $a_{i j}$ are coordinates on $\mathbb{K}^{N}$. Thus fibers of integrating foliations $\mathcal{F}_{\lambda}$ on $\mathcal{B}$ are parallel planes in $\mathbb{K}^{N}$, hence the Kronecker web structure on $\mathcal{B}$ is translation-invariant.

To finish the proof of the theorem, it is enough to recall [5] that reductive Lie algebras satisfy the lemma.

Corollary 14.24. In conditions of Theorem 14.22 there is an open dense subset $U \subset \mathfrak{g}^{*}$ such that the restriction on $U$ of the bihamiltonian structure is locally isomorphic to a direct product of several copies of the structure of Example 0.9.

Proof. It is enough to show that a micro-Kronecker translation-invariant bihamiltonian structure can be represented as a product of structures of Example 0.9. This a direct corollary of Theorem 5.1.

Sum up conditions under which the statements about flatness can be achieved (at least in a weak form).

Definition 14.25. Consider an open subset $U \subset V$ in a vector space $V$, a foliation $\mathcal{F}$ of codimension $r$ on $U$, and an involution $\psi$ of $U$. Call $\mathcal{F}$ compatible with $\psi$ if

1. The leaves of $\mathcal{F}$ are common level sets of polynomials $p_{1}, \ldots, p_{r}$ on $U$;

2. Differentials $d p_{i}$ are linearly independent at any point of $U$;

3. $2 \sum_{i=1}^{r} \operatorname{deg} p_{i}+r=\operatorname{dim} V$;

4. The submanifold Fix $(\psi)$ of fixed points of $\psi$ in $U$ is nonempty and is transversal to leaves of $\mathcal{F}$. 
The arguments we had so far lead to

Corollary 14.26. Consider an anti-involution $\iota$ of a 2-regular Lie algebra $\mathfrak{g}$. Suppose that there is an open subset $U \subset \mathfrak{g}^{*}$ such that the $\mathrm{Ad}^{*}$-orbits in $U$ form a foliation which is compatible with the involution $\iota^{*}$ of $\mathfrak{g}^{*}$. Then there is an open subset $U_{1} \subset \mathfrak{g}^{*}$, and for any $c_{1} \in U_{1}$ there is an open subset $U_{2}\left(c_{1}\right) \subset \mathfrak{g}^{*}$ on which the bihamiltonian structure associated to $c_{1}$ is flat.

This reduces the question of flatness to a question on geometry of $\mathrm{Ad}^{*}$-action on $\mathfrak{g}^{*}$. Let us sketch roughly how to construct new Lie algebras which satisfy the conditions of the corollary.

First of all, if $\mathfrak{g}$ satisfies these condition, then $\mathfrak{g}^{n}$ satisfies these conditions too. The subset $U$ should be replaced by $U^{n}$, and $\iota$ by $\iota^{n}$. More generally, if $Z$ is a set, or a topological space, or a manifold, or a variety, or a scheme, then a following variation is possible. Denote by $\mathfrak{g}^{Z}$ the set (or topological space, etc) of mappings from $Z$ to $\mathfrak{g}$. It has a natural Lie algebra structure, and it acts on $\left(\mathfrak{g}^{*}\right)^{Z}$. Moreover, $\left(\mathfrak{g}^{*}\right)^{Z}$ has an involution $\iota^{Z}$, and in many situations $U^{Z}$ makes sense ${ }^{38}$ as well.

Suppose that $U^{Z}$ makes sense, and $G^{Z}$ makes sense too (here $G$ is a Lie group with the Lie algebra $\mathfrak{g})$. Then the orbits of action of $G^{Z}$ on $\left(\mathfrak{g}^{*}\right)^{Z}$ can be described in the same way as in the case of $\mathfrak{g}^{n}$ : given a linear functional $\varepsilon$ on functions on $Z$ and an invariant polynomial $p$ on $U$, define a $G^{Z}$-invariant function $p_{\varepsilon}$ on $U^{Z}$ by $p_{\varepsilon}(f)=\langle\varepsilon, p \circ f\rangle$; here $f: Z \rightarrow U$. If $Z$ is "small" 39 intersections of $G^{Z}$-orbits with $U^{Z}$ coincide with common level sets of functions $p_{\varepsilon}$ on $U^{Z}$. Thus all the needed qualitative properties of orbits of $G^{Z}$ on $U^{Z}$ hold in this case if they hold for the action of $\mathfrak{g}$ on $U$.

The property $2 \sum_{i=1}^{r} \operatorname{deg} p_{i}+r=\operatorname{dim} V$ makes no sense if $\mathfrak{g}^{Z}$ is infinite-dimensional, but holds if $\mathfrak{g}^{Z}$ is finite-dimensional. We conclude:

Corollary 14.27. Suppose that a Lie algebra $\mathfrak{g}$ with an anti-involution $\iota$ satisfies conditions of Corollary 14.26. If $A$ is a finite-dimensional commutative algebra, and $Z=$ Spec $A$, then the orbits of action of $G^{Z}$ on $\left(\mathfrak{g}^{*}\right)^{Z}$ are compatible with $\iota^{Z}$ on an appropriate open subset of $G^{Z}$ (here $G$ is the Lie group for $\mathfrak{g}$ ). Moreover, $\mathfrak{g}^{Z}$ is 2-regular.

Proof. The only thing to prove is that $\mathfrak{g}^{Z}$ is 2-regular. Constant mappings give an inclusion $\mathfrak{g} \hookrightarrow \mathfrak{g}^{Z}$, and regular elements of $\mathfrak{g}$ go to regular elements of $\mathfrak{g}^{Z}$. This shows that $\mathbb{P} \mathfrak{g}$ intersects irregular elements of $\mathbb{P}^{Z}$ over a subset of codimension 2 or more, thus $\mathfrak{g}^{Z}$ is 2-regular.

Note that this statement does not allow us to show that $\mathfrak{g}^{Z}$ satisfies conditions of Corollary 14.26 , since $\left(\mathfrak{g}^{Z}\right)^{*}$ is not necessarily related to $\left(\mathfrak{g}^{*}\right)^{Z}$. However, if $A^{*}$ is

\footnotetext{
${ }^{38}$ If $Z$ is an affine line, and $U$ has many "holes" (complement to $U$ is large), then the set of algebraic mappings to $U$ can consist of constant mappings only. We want to avoid such a situation.

${ }^{39}$ It looks like it is enough to require that any étale covering of $Z$ can be refined to a usual covering.
} 
isomorphic to $A$ as an $A$-module, then the action of $G^{Z}$ on $\left(\mathfrak{g}^{Z}\right)^{*}$ is isomorphic to the action of $G^{Z}$ on $\left(\mathfrak{g}^{*}\right)^{Z}$.

If $Z$ is infinitesimally small, then the sketches of arguments outlined above can be made precise. This leads to the following:

Theorem 14.28. If a Lie algebra $\mathfrak{g}$ with an anti-involution $\iota$ satisfies conditions of Corollary 14.26, and $A$ is a finite-dimensional commutative algebra such that $A$ module $A$ is self-dual, and $Z=$ Spec $A$, then $\mathfrak{g}^{Z}$ satisfies conditions of Corollary 14.26.

Example 14.29. $A_{a_{1} \ldots a_{k}}=\mathbb{K}\left[z_{1}, \ldots, z_{k}\right] / z_{1}^{a_{1}+1} \ldots z_{k}^{a_{k}+1}$ is self-dual. Let $Z_{a_{1} \ldots a_{k}}=$ Spec $A_{a_{1} \ldots a_{k}}$. Then $Z_{a_{1} \ldots a_{k}}=Z_{a_{1}} \times \cdots \times Z_{a_{k}}$, thus $\mathfrak{g}^{Z_{a_{1} \ldots a_{k}}}=\left(\left(\mathfrak{g}^{Z_{a_{1}}}\right)^{\cdots}\right)^{Z_{a_{k}}}$. In other words, the construction above with $Z=Z_{a_{1} \ldots a_{k}}$ is equivalent to a repeated $k$ times construction with $Z=Z_{a_{l}}, l=1, \ldots, k$.

Example 14.30. For a different example of the self-dual case consider

$$
B_{k}=\mathbb{K}\left[z_{1}, z_{2}\right] /\left(z_{1} z_{2}, z_{1}^{k}-z_{2}^{k}\right) .
$$

There is a relation between $B_{k}$ and $A_{k} \times A_{k}=\mathbb{K}\left[z_{1}\right] / z_{1}^{k+1} \times \mathbb{K}\left[z_{2}\right] / z_{2}^{k+1}$. Let $C_{k}=$ $A_{k} \times A_{k} /\left(\left(z_{1}^{k},-z_{2}^{k}\right)\right)$. Then $B_{k}$ can be included into $C_{k}$ by $z_{1} \mapsto\left(z_{1}, 1\right), z_{2} \mapsto\left(1, z_{2}\right)$, $1 \rightarrow(1,1)$.

The scheme $\operatorname{Spec} A_{k} \times A_{k}$ coincides with $Z_{k} \coprod Z_{k}$. Thus $Y_{k} \stackrel{\text { def }}{=} \operatorname{Spec} C_{k}$ is a subscheme of $Z_{k} \coprod Z_{k}$, and $X_{k} \stackrel{\text { def }}{=} \operatorname{Spec} B_{k}$ is a quotient of $Y_{k}$. The usual "picture" of $Z_{k}$ is an "interval of infinitesimal length" $k$. It is hard to picture $C_{k}$ : it should be a subscheme of two copies of such an interval, but the sense of the equation $z_{1}^{k}-z_{2}^{k}$ of $Y_{k}$ inside $Z_{k} \coprod Z_{k}$ indicates visualization via gluing, not via cutting-off unnecessary parts: indeed, the function $z^{k}$ vanishes on a subinterval $Z_{k-1}$ of $Z_{k}$, thus in some sense $z^{k}$ is non-zero only at the "end" of $Z_{k}$. Thus in some sense the functions on $Y_{k}$ "coincide" with functions on $Z_{k} \coprod Z_{k}$ which have the same values at the "ends" of two copies of $Z_{k}$. On the other hand, functions on $X_{k}$ are exactly the functions on $Y_{k}$ values of which at two centers of two copies of $Z_{k}$ coincide.

Thus $X_{k}$ may be visualized as two infinitesimal intervals $Z_{k}$ glued both at the centers and at the "end" (here gluing of the centers is done in a precise algebro-geometric sense, gluing of ends - and ends themselves - exist only as a figure of speech). This suggests an analogy of $X_{k}$ with a kind of "infinitesimal loop". As the results above show, $\mathfrak{g}^{X_{k}}$ has "nice" properties if $\mathfrak{g}$ does. It would be interesting to compare properties of $\mathfrak{g}^{X_{k}}$ with properties of the Lie algebra $\widehat{\mathfrak{g}}$ of loops in $\mathfrak{g}$.

Example 14.31. Consider $Z=Z_{1}$. Clearly, $\mathfrak{g}^{Z_{1}}=\mathfrak{g} \ltimes \mathrm{ad}_{\mathfrak{g}}$; here $\operatorname{ad}_{\mathfrak{g}}$ is the commutative Lie algebra which coincides with $\mathfrak{g}$ as a vector space, and with adjoint action of $\mathfrak{g}$. If $\mathfrak{g}$ is reductive, then $\mathfrak{g}^{Z_{1}} \simeq \mathfrak{g} \ltimes \mathrm{ad}_{\mathfrak{g}}^{*}$ as well. This shows that in the situation of Example 14.10 the bihamiltonian structure is flat on an open subset if $c_{1}$ belongs to an open subset.

Consider now a different point of view on some of the results of this section. 
Remark 14.32. It is instructive to compare the statement of Proposition 14.19 with the statement of Theorem 5.1. Let $\mathfrak{g}=\mathfrak{g l}(n, \mathbb{C}), \iota$ be the Cartan anti-involution. Then $-\iota$ is an involution. The fixed points of $-\iota$ form $\mathfrak{o}(n)$, or linear transformations preserving a non-degenerate symmetric bilinear form $\alpha(v, w)$ in $\mathbb{C}^{n}$. The anti-involutions which differ from $\iota$ by a conjugation will lead to equivalent bilinear forms. Note that any two non-degenerate symmetric bilinear form in $\mathbb{C}^{n}$ are equivalent.

Fixed points of $\iota$ are symmetric matrices. Given such a matrix $X$, consider the form $\beta(v, w)=\alpha(X v, w)$. It is a symmetric bilinear form, any symmetric bilinear form can be written in this way for an appropriate $X$. A bilinear form $\gamma$ in $V$ can be considered as mappings $\widetilde{\gamma}: V \rightarrow V^{*}:\langle\widetilde{\gamma} v, w\rangle=\gamma(v, w)$. Let $\widetilde{\alpha}, \widetilde{\beta}$ be mappings associated to $\alpha$ and $\beta$. Then $X=\widetilde{\alpha} \widetilde{\beta}^{-1}$. Call $X$ the associated operator for bilinear forms $\alpha, \beta$.

Now the statement of Proposition 14.19 in the case $\mathfrak{g}=\mathfrak{g l}(n, \mathbb{C})$ can be read as follows: any operator $X$ in $n$-dimensional complex vector space $V$ is ${ }^{40}$ an associated operator of two symmetric bilinear forms $\alpha, \beta$ (here $\alpha$ is non-degenerate). On the other hand, if one does the same for skew-symmetric bilinear forms, then by Theorem 5.1 the Jordan blocks $X$ would come in pairs.

\section{Appendix On KRONECKER DECOMPOSITIONS}

We know that any Kronecker relation $W$ in a vector space $V$ can be decomposed into a direct sum of Kronecker blocks $W_{i}$ in subspaces $V_{i} \subset V$. However, this decomposition is not unique. Here we sketch the degree of arbitrariness of this decomposition.

Given such a decomposition $V=\bigoplus V_{i}$, consider the following objects:

Definition 15.1. The isotypic component $\mathcal{I}_{k}(V)$ of type $k$ of a decomposition $V=$ $\bigoplus V_{i}$ is the sum of subspaces $V_{i}$ of dimension $k$. The isotypic filtration $F_{k}$ of a decomposition $V=\bigoplus V_{i}$ is $F_{k} V=\sum_{l \leq k} \mathcal{I}_{l}(V)$.

A vector subspace $S \subset V$ is a $k$-isotypic block if there is a decomposition $V=\bigoplus V_{i}$ such that $S$ is the isotypic component of type $k$.

Theorem 15.2. The isotypic filtration of a Kronecker relation in $V$ does not depend on the choice of a decomposition $V=\bigoplus V_{i}$ into Kronecker blocks.

Proof. Start with

Definition 15.3. Given a decomposition $V=\bigoplus V_{i}$ of a relation $W$ in $V$ into Kronecker blocks and $\lambda \in \mathbb{K} \mathbb{P}^{1}$, let $\lambda$-filtration be the filtration $F_{k} \operatorname{Ker}_{\lambda} W$ of $\operatorname{Ker}_{\lambda} W$ by $\operatorname{Ker}_{\lambda} W \cap F_{k} V$.

\footnotetext{
${ }^{40}$ Contrast this with the real case and the case of positive $\alpha$ : then any associated operator is diagonalizable!
} 
Due to Lemma 6.3 , if $\lambda_{i}, i \geq 0$, is a sequence of different elements of $\mathbb{K P}^{1}$, then $F_{k} V=\sum_{i=1}^{k} F_{k} \operatorname{Ker}_{\lambda_{i}} W$. Thus it is enough to show that the $\lambda$-filtration in $\operatorname{Ker}_{\lambda} W$ does not depend on the choice of decomposition into Kronecker blocks.

Now Lemmas 6.3 and 7.3 taken together imply that $F_{k} \operatorname{Ker}_{\lambda_{0}} W=\operatorname{Ker}_{\lambda_{0}} W \cap$ $\sum_{i=1}^{k} \operatorname{Ker}_{\lambda_{i}} W$. Thus $F_{k} \operatorname{Ker}_{\lambda_{0}} W$ does not depend on the choice of decomposition.

Remark 15.4. Note that this theorem implies the statement of Theorem 3.7 about uniqueness of the collection of dimension of Kronecker blocks in the decomposition of a given Kronecker relation.

Obviously, a choice of a decomposition of a given isotypic component into Kronecker blocks is extremely non-unique if the component has more than one block.

Proposition 15.5. Consider a Kronecker relation $W$ in $V$, suppose that all the Kronecker blocks of $W$ have the same dimension. Fix $\lambda_{0} \in \mathbb{K}^{1}$. Given a decomposition $V=\bigoplus V_{i}$ into Kronecker blocks $V_{i}$ with relations $W_{i}$, one obtains a decomposition $\operatorname{Ker}_{\lambda_{0}} W=\bigoplus \operatorname{Ker}_{\lambda_{0}} W_{i}$ into a direct sum sum of 1-dimensional subspaces.

Given an arbitrary decomposition $\operatorname{Ker}_{\lambda_{0}} W=\bigoplus Y_{i}$ into one-dimensional subspaces, one can find a decomposition $V=\bigoplus V_{i}$ into Kronecker blocks such that $\operatorname{Ker}_{\lambda_{0}} V_{i}=Y_{i}$. The subspace $V_{i}$ is uniquely determined by the subspace $Y_{i}$.

Proof. Suppose that dimensions of Kronecker blocks of $V$ are equal to $k$. Let $\Lambda \subset \mathbb{K P}^{1}$ has $k+1$ element, let $K_{\lambda}=\operatorname{Ker}_{\lambda} W, \lambda \in \Lambda$. By Amplification 7.2 the collection $\left\{K_{\lambda}\right\}_{\lambda \in \Lambda}$ uniquely determines $W$. Express possible decompositions of $W$ into Kronecker blocks in terms of this collection.

Suppose that $\Lambda=\Lambda_{0} \cup\{\tilde{\lambda}\}, \widetilde{\lambda} \notin \Lambda_{0}$. By Lemmas 6.3, 7.3,V= $\bigoplus_{\lambda \in \Lambda_{0}} K_{\lambda}$, denote by $\pi_{\lambda}, \lambda \in \Lambda_{0}$, the projection of $V$ on $K_{\lambda}$ according to this decomposition. As one can easily check, $\pi_{\lambda} K_{\tilde{\lambda}} \neq 0$, thus $\pi_{\lambda} K_{\tilde{\lambda}}=K_{\lambda}$, provided $\lambda \in \Lambda_{0}$ and $V$ is a Kronecker block with $\operatorname{dim} V=k$. Hence $\pi_{\lambda} K_{\tilde{\lambda}}=K_{\lambda}$ for an arbitrary $k$-isotypic $V$. We see that projections $\pi_{\lambda}$ identify all the $K_{\lambda}$ with $K_{\tilde{\lambda}}$, thus one with another.

Assume $\lambda_{0} \in \Lambda_{0}$. Due to the identifications above, a choice of a basis $v_{i}^{\left(\lambda_{0}\right)}$ in $K_{\lambda_{0}}$ induces bases $v_{i}^{(\lambda)}$ in each of the subspaces $K_{\lambda}, \lambda \in \Lambda_{0}$, thus a basis in $V$. Let $V_{i}$ is spanned by $v_{i}^{(\lambda)}, \lambda \in \Lambda_{0}$.

Lemma 15.6. Consider a vector space $\widetilde{V}, \operatorname{dim} \widetilde{V}=k$, and one-dimensional subspaces $T_{\lambda} \subset \widetilde{V}, \lambda \in \Lambda \subset \mathbb{K P}^{1}$, card $(\Lambda)=k+1$. Suppose that each collection of $k$ subspaces out of $\left\{T_{\lambda}\right\}$ spans the whole vector space. Then there is one and only one Kronecker relation $\widetilde{W}$ in $\widetilde{V}$ such that $\operatorname{Ker}_{\lambda} \widetilde{W}=T_{\lambda}, \lambda \in \Lambda$,

Proof. The "only one" part follows from Amplification 7.2. On the other hand, if $W_{0}$ is a Kronecker block in $V_{0}, \operatorname{dim} V_{0}=k$, then there is (exactly one up to proportionally) linear mapping $f$ from $V_{0}$ to $\widetilde{V}$ such that $f\left(\operatorname{Ker}_{\lambda} W\right)=T_{\lambda}, \lambda \in \Lambda$. Since $f$ is invertible, putting $\widetilde{W}=f_{!} W_{0}$ finishes the proof. 
Apply the lemma to $\widetilde{V}=V_{i}, T_{\lambda}=V_{i} \cap K_{\lambda}, \lambda \in \Lambda$. By the construction of the basis $v_{i}^{(\lambda)}, T_{\lambda}$ is one-dimensional, thus the conditions of the lemma apply. This gives a Kronecker-block linear relation $\widetilde{W}_{i}$ in $V_{i}$. Then $\widetilde{W}=\bigoplus \widetilde{W}_{i}$ is a Kronecker linear relation in $V$ with all the Kronecker blocks having dimension $k$, and $\operatorname{Ker}_{\lambda} \widetilde{W}=K_{\lambda}$ for $\lambda \in \Lambda$. By Amplification 7.2,W= $W$, thus $\bigoplus \widetilde{W}_{i}$ is the required decomposition of $W$ into a direct sum of Kronecker blocks.

Due to Theorem 15.2, the only arbitrariness in the choice of $k$-isotypic block is the choice of an appropriate complement of $F_{k-1} V$ in $F_{k} V$. Study which complements may appear as isotypic blocks.

To simplify notations we may assume that $V=F_{k} V$ (call such $W$ a relation of type $\leq k)$. This assumption holds until the end of this section.

Definition 15.7. Given a $k$-isotypic block $S \subset V$ of a Kronecker relation $W$ of type $\leq k$ in $V$, let $\lambda$-pivot space of $S$ be $S \cap \operatorname{Ker}_{\lambda} W$; here $\lambda \in \mathbb{K} \mathbb{P}^{1}$.

Lemma 15.8. Suppose that $W$ is a relation of type $\leq k$ in $V$. Then a $\lambda$-pivot space is a complement to $F_{k-1} \operatorname{Ker}_{\lambda} W$ in $\operatorname{Ker}_{\lambda} W$.

Proof. This follows directly from decomposability into Kronecker blocks.

Definition 15.9. Consider 3 subspaces $V, V^{\prime}, V^{\prime \prime}$ of a vector space $W$. Say that $V^{\prime} \equiv V^{\prime \prime} \bmod V$ if $\operatorname{dim} V^{\prime}=\operatorname{dim} V^{\prime \prime}$ and images of $V^{\prime}$ and $V^{\prime \prime}$ in $W / V$ coincide.

Definition 15.10. Consider a finite subset $\Lambda \subset \mathbb{K}^{1}$ and a Kronecker relation $W$ in $V$ of type $\leq k$. A collection of vector subspaces $S_{\lambda} \subset \operatorname{Ker}_{\lambda} W, \lambda \in \Lambda$, is admissible if there is a $k$-isotypic block $S$ such that $S_{\lambda}=S \cap \operatorname{Ker}_{\lambda} W$ for any $\lambda \in \Lambda$.

Call $\left\{S_{\lambda}\right\}_{\lambda \in \Lambda} l$-admissible if there is a $k$-isotypic block $S$ such that $S_{\lambda} \equiv S \cap \operatorname{Ker}_{\lambda} W$ $\bmod F_{l} \operatorname{Ker}_{\lambda} W$ for any $\lambda \in \Lambda$.

In particular, a collection $\left\{S_{\lambda}\right\}_{\lambda \in \Lambda}$ is $k$-1-admissible iff $S_{\lambda}$ is a complement to $F_{k-1} \operatorname{Ker}_{\lambda} W$ in $\operatorname{Ker}_{\lambda} W$.

Definition 15.11. A sequence $v_{1}, \ldots, v_{l}$ of elements of $V$ forms a $W$-chain if for any two consecutive elements $v, \widetilde{v}$ of the sequence $0, v_{1}, \ldots, v_{l}, 0$ the pair $(v, \widetilde{v}) \in W$.

Consider the pencil $\mathcal{P}_{1}, \mathcal{P}_{2}: V \rightarrow V^{\prime}$ which corresponds to $W$ as in Section 2. It is clear that $v_{1}, \ldots, v_{l}$ forms a $W$-chain iff $v_{1}+\lambda v_{2}+\cdots+\lambda^{l-1} v_{l}$ is in the kernel of $\lambda \mathcal{P}_{1}-\mathcal{P}_{2}$ (here we consider $\lambda$ as a new variable, thus the relation holds over $\mathbb{K}[\lambda]$ ). Each Kronecker block $S_{i}$ of dimension $l$ in $V$ with a basis $\boldsymbol{f}_{1}^{(i)}, \ldots, \boldsymbol{f}_{l}^{(i)}$ (as in Example 3.3) gives a $W$-chain $\boldsymbol{f}_{1}^{(i)}, \ldots, \boldsymbol{f}_{l}^{(i)}$.

Definition 15.12. Given two $W$-chains $v_{1}, \ldots, v_{l}$ and $v_{1}^{\prime}, \ldots, v_{m}^{\prime}, m<l$, define the $n$-th elementary operation as a change of $v_{i}$ to $v_{i}+C v_{i-n}^{\prime}$. Here $C \in \mathbb{K}, 0 \leq n \leq l-m$, and we extend the sequence $v_{i}^{\prime}$ to $i \leq 0$ and $i>m$ by 0 . The elementary operation of the first kind is the 0 -th elementary operation, elementary operation of the second kind is the $l-m$-th elementary operation. 
Note that an elementary operation transforms a $W$-chain into a $W$-chain, and that the operations of the first kind do not change $v_{l}$, while operations of the second kind do not change $v_{1}$.

Remark 15.13. Consider a $k$-isotypic block $S$ of $V$. Taking a $W$-chain $v_{i}$ corresponding to a Kronecker block of $S$, and a $W$-chain $v_{i}^{\prime}$ corresponding to a Kronecker block of $F_{k-1} V$, one can perform elementary operations using these chains. These operations will change the chain $v_{i}$. The following lemma implies that this change corresponds to a change of the $k$-isotypic block $S$ into another $k$-isotypic block:

Lemma 15.14. Suppose that vectors $v_{i j} \in L, 1 \leq i \leq k, j \in J$, are linearly independent, span a complement $S$ to $F_{k-1} V$ in $V$, and for any $j \in J$ the sequence $v_{i j}, 1 \leq i \leq k$, is a $W$-chain in $V$. Then $S$ is a $k$-isotypic block in $V$.

Proof. Consider again the pencil $\mathcal{P}_{1}, \mathcal{P}_{2}: V \rightarrow V^{\prime}$. It is clear that $\mathcal{P}_{1} S=\mathcal{P}_{2} S$ as subspaces in $V^{\prime}$. If we prove that $\mathcal{P}_{1}\left(F_{k-1} V\right)$ (which coincides with $\mathcal{P}_{2}\left(F_{k-1} V\right)$ ) does not intersect $\mathcal{P}_{1} S$, then one can split $S$ and $\mathcal{P}_{1} S$ into direct summands (in $V$ and $V^{\prime}$ correspondingly), which will prove the lemma.

Suppose that $\mathcal{P}_{1}\left(F_{k-1} V\right)$ does intersect $\mathcal{P}_{1} S$. Consider an arbitrary $k$-isotypic block $S^{\prime}$ in $V$. Conditions of the lemma imply that $S \equiv S^{\prime} \bmod F_{k-1} V$. This implies that $\mathcal{P}_{1}\left(F_{k-1} V\right)$ intersects $\mathcal{P}_{1} S^{\prime}$, which is a contradiction.

Definition 15.15. Given a $k$-isotypic block $S$ of $V$, call the modifications of $S$ resulting from elementary operations of Remark 15.13 the elementary operations over $k$-isotypic blocks.

Obviously:

Lemma 15.16. Using elementary operations of the first kind one can change $S \cap$ $\mathrm{Ker}_{0: 1} W$ to become an arbitrary complement to $F_{k-1} \operatorname{Ker}_{0: 1} W$ in $\operatorname{Ker}_{0: 1} W$ without changing $S \cap \operatorname{Ker}_{1: 0} W$. Similarly, the operations of the second kind will do the same with $S \cap \operatorname{Ker}_{1: 0} W$.

This implies

Lemma 15.17. Fix $\lambda^{\prime}, \lambda^{\prime \prime} \in \mathbb{K P}^{1}, \lambda^{\prime} \neq \lambda^{\prime \prime}$. Suppose that $V=F_{k} V$. Then any $k-1$-admissible pair of subspaces $\left(S_{\lambda^{\prime}}, S_{\lambda^{\prime \prime}}\right)$ is admissible.

Moreover, one can improve this statement by considering subsets $\Lambda \subset \mathbb{K P}^{1}$ with more than two elements. Also, one can describe the degree of arbitrariness in the choice of an isotypic block $S$ with given intersections $S \cap \operatorname{Ker}_{\lambda} W$ for $\lambda \in \Lambda$. Start with the following

Proposition 15.18. Consider two ${ }^{41} k$-isotypic blocks $S$ and $S^{\prime}$ in $V$. There is a sequence of elementary operations which transforms $S$ into $S^{\prime}$.

\footnotetext{
${ }^{41}$ These blocks would correspond to different decomposition of $V$ into direct sums of Kronecker blocks.
} 
Proof. By Lemma 15.16, one may suppose that $S \cap \mathrm{Ker}_{0: 1} W=S^{\prime} \cap \mathrm{Ker}_{0: 1} W$. Consider $W$-chains which form bases in $S$ and $S^{\prime}$. Denote these chains in $S$ by $v_{i, j}$, in $S^{\prime}$ by $v_{i, j}^{\prime}$ (here $j$ enumerates chains, and $i$ vectors inside a chain). By Proposition 15.5 we may assume that $v_{1, j}=v_{1, j}^{\prime}$. Let $p_{j}=\sum_{i} v_{i j} \lambda^{i-1}$ be the polynomial in $\lambda$ which corresponds to the $W$-chain $v_{\bullet}, j$, similarly introduce $p_{j}^{\prime}$. Fix $j$. Obviously, $p_{j}^{\prime}-p_{j}$ can be written as $\lambda q$, and the polynomial $q$ corresponds to an appropriate $W$-chain $\widetilde{v}_{i}$. Moreover, all the vectors $\widetilde{v}_{i}$ are in $F_{k-1} V$.

Decompose $F_{k-1} V$ into direct sum of Kronecker components, consider projections of $\widetilde{v}_{i}$ to these components. Clearly, for any such projection $\pi_{\alpha}$ the vectors $\pi_{\alpha} \widetilde{v}_{i}$ form a $W$-chain. Now the proposition follows from the following

Lemma 15.19. Consider a Kronecker block $\widetilde{W}$ in $\widetilde{V}$ and a $\widetilde{W}$-chain $v_{i}$ which is a basis of $\widetilde{V}$. Let $v_{i}^{\prime}$ be an arbitrary $\widetilde{W}$-chain in $\widetilde{V}$. Let $p=\sum_{i} v_{i} \lambda^{i-1}, p^{\prime}=\sum_{i} v_{i}^{\prime} \lambda^{i-1}$. Then there is a polynomial $q \in \mathbb{K}[\lambda]$ such that $p^{\prime}=q p$.

Proof. Write $v_{j}^{\prime}$ in the basis $v_{i}$ and compare the coefficients using the definition of a $W$-chain.

This finishes proof of the proposition.

From this proposition one can immediately deduce

Theorem 15.20. Let $S$ be a k-isotypic block in $V$, and $\Lambda \subset \mathbb{K P}^{1}$.

1. Let $S^{\prime}$ be another $k$-isotypic block in $V$. Suppose that $l<k$,

$$
S \cap \operatorname{Ker}_{\lambda} W \equiv S^{\prime} \cap \operatorname{Ker}_{\lambda} W \bmod F_{l} \operatorname{Ker}_{\lambda} W \text { for } \lambda \in \Lambda .
$$

If $\operatorname{card}(\Lambda)=k-l$, then

$$
S \cap \operatorname{Ker}_{\lambda} W \equiv S^{\prime} \cap \operatorname{Ker}_{\lambda} W \bmod F_{l} \operatorname{Ker}_{\lambda} W \text { for any } \lambda .
$$

2. Suppose that for $\lambda \in \Lambda$ a vector subspace $S_{\lambda}^{\prime} \subset \operatorname{Ker}_{\lambda} W$ is fixed, and $S_{\lambda}^{\prime} \equiv$ $S \cap \operatorname{Ker}_{\lambda} W \bmod F_{l+1} \operatorname{Ker}_{\lambda} W$ for $\lambda \in \Lambda$. Then if card $(\Lambda)=k-l$, then there exists another $k$-isotypic block $S^{\prime}$ in $V$ such that

$$
S_{\lambda}^{\prime} \equiv S^{\prime} \cap \operatorname{Ker}_{\lambda} W \bmod F_{l} \operatorname{Ker}_{\lambda} W
$$

This theorem gives a complete description of the arbitrariness in the choice of the $k$-isotypic block in $V$. For example, consider a subset $\left\{\lambda_{i}\right\}$ of $\mathbb{K} \mathbb{P}^{1}$. Subspaces $S \cap \operatorname{Ker}_{\lambda_{1}} W$ and $S \cap \operatorname{Ker}_{\lambda_{2}} W$ (which may be arbitrary complements to $F_{k-1} \operatorname{Ker}_{\lambda_{i}} W$ in $\left.\operatorname{Ker}_{\lambda_{i}} W, i=1,2\right)$ completely determine $S \bmod F_{k-2}$. In particular, they determine $S \cap \operatorname{Ker}_{\lambda_{3}} W \bmod F_{k-2} \operatorname{Ker}_{\lambda_{3}} W$. A choice of an arbitrary subspace $S_{\lambda_{3}}$ of $\operatorname{Ker}_{\lambda_{3}} W$ with the same reduction $\bmod F_{k-2} \operatorname{Ker}_{\lambda_{3}} W$ completely determines $S \bmod F_{k-3}$ by requiring $S \cap \operatorname{Ker}_{\lambda_{2}} W=S_{\lambda_{3}}$, etc., etc., etc. Together with Proposition 15.5 this describes all possible decompositions of $V$ into Kronecker blocks. 


\section{REFERENCES}

1. J. Frank Adams, Lectures on Lie groups, W. A. Benjamin, Inc., New York-Amsterdam, 1969.

2. V. I. Arnol'd, Mathematical methods of classical mechanics, Springer-Verlag, New York, 199?, Translated from the 1974 Russian original by K. Vogtmann and A. Weinstein, Corrected reprint of the second (1989) edition.

3. V. I. Arnol'd and A. B. Givental', Symplectic geometry, Current problems in mathematics. Fundamental directions, Vol. 4, Akad. Nauk SSSR Vsesoyuz. Inst. Nauchn. i Tekhn. Inform., Moscow, 1985, pp. 5-139, 291.

4. A. V. Bolsinov, Compatible Poisson brackets on Lie algebras and the completeness of families of functions in involution, Izv. Akad. Nauk SSSR Ser. Mat. 55 (1991), no. 1, 68-92.

5. N. Bourbaki, Groupes et algebres de Lie, Herman, Paris, 1975, Ch7-8.

6. E. B. Dynkin, Maximal subgroups of the classical groups, Trudy Moskov. Mat. Obšč. 1 (1952), 39-166.

7. L. D. Faddeev and L. A. Takhtajan, Hamiltonian methods in the theory of solitons, SpringerVerlag, Berlin, 1987, Translated from the Russian by A. G. Reyman [A. G. Reimman].

8. A. S. Fokas and B. Fuchssteiner, On the structure of symplectic operators and hereditary symmetries, Lett. Nuovo Cimento (2) 28 (1980), no. 8, 299-303.

9. F. R. Gantmacher, The theory of matrices. Vols. 1, 2, Chelsea Publishing Co., New York, 1959, Translated by K. A. Hirsch.

10. I. M. Gel'fand and I. Ja. Dorfman, Hamiltonian operators and algebraic structures associated with them, Funktsional. Anal. i Prilozhen. 13 (1979), no. 4, 13-30, 96.

11. Israel M. Gelfand and Ilya Zakharevich, Webs, Lenard schemes, and the local geometry of bihamiltonian Toda and Lax structures, Archived as math.DG/9903080. To appear in Selecta Math.

12. (1989), no. 1, 85-93.

13. _ Webs, Veronese curves, and bihamiltonian systems, J. of Func. Anal. 99 (1991), 150178.

14. 1990-1992 (Boston), Birkhäuser, 1993, pp. 51-112.

15. _ The spectral theory for a pencil of skewsymmetrical differential operators of the third order, Comm. Pure Appl. Math. 47 (1994), no. 8, 1031-1041.

16. Victor Guillemin and Shlomo Sternberg, Geometric asymptotics, American Mathematical Society, Providence, R.I., 1977, Mathematical Surveys, No. 14.

17. A. A. Kirillov, Local Lie algebras, Uspehi Mat. Nauk 31 (1976), no. 4(190), 57-76.

18. Y. Kosmann-Schwarzbach and F. Magri, Lax-Nijenhuis operators for integrable systems, J. Math. Phys. 37 (1996), no. 12, 6173-6197.

19. Bertram Kostant, The solution to a generalized Toda lattice and representation theory, Adv. in Math. 34 (1979), no. 3, 195-338.

20. Peter D. Lax, Almost periodic solutions of the KdV equation, SIAM Rev. 18 (1976), no. 3, $351-375$.

21. Franco Magri, A simple model of the integrable Hamiltonian equation, Journal of Mathematical Physics 19 (1978), no. 5, 1156-1162.

22. - On the geometry of soliton equations, Preprint, 1988.

23. $\ldots$ On the geometry of soliton equations, Acta Appl. Math. 41 (1995), no. 1-3, 247-270, Geometric and algebraic structures in differential equations.

24. Henri McKean, private communication, 1990. 
25. A. S. Miščenko and A. T. Fomenko, Euler equation on finite-dimensional Lie groups, Izv. Akad. Nauk SSSR Ser. Mat. 42 (1978), no. 2, 396-415, 471.

26. Carlo Morosi and Livio Pizzocchero, On the Euler equation: bi-Hamiltonian structure and integrals in involution, Lett. Math. Phys. 37 (1996), no. 2, 117-135.

27. Andriy Panasyuk, Symplectic realizations of bihamiltonian structures, preprint, 1998.

28. _ Veronese webs for bihamiltonian structures of higher corank, Banach Center Publications, Poisson geometry, Proceedings of the conference dedicated to the memory of Stanislaw Zakrzewski, Warsaw 1998 (Warszawa) (Pawel Urbański and Janusz Grabowski, eds.), Instytut Matematyczny PAN, 1999, to appear.

29. Marie-Hélène Rigal, Géométrie globale des systèmes bihamiltoniens réguliers de rang maximum en dimension 5, C. R. Acad. Sci. Paris Sér. I Math. 321 (1995), no. 11, 1479-1484.

30. Tissus de Véronèse en dimension 3, Séminaire Gaston Darboux de Géométrie et Topologie Différentielle, 1994-1995 (Montpellier), Univ. Montpellier II, Montpellier, 1995, pp. iv, 63-68.

31. _ Systèmes bihamiltoniens en dimension impaire, Ann. Sci. École Norm. Sup. (4) 31 (1998), no. 3, 345-359.

32. Robert C. Thompson, Pencils of complex and real symmetric and skew matrices, Linear Algebra Appl. 147 (1991), 323-371.

33. Francisco-Javier Turiel, Classification locale d'un couple de formes symplectiques Poissoncompatibles, Comptes Rendus des Seances de l'Academie des Sciences. Serie I. Mathematique 308 (1989), no. 20, 575-578.

34. $\_, C^{\infty}$-équivalence entre tissus de Veronese et structures bihamiltoniennes, C. R. Acad. Sci. Paris Sér. I Math. 328 (1999), no. 10, 891-894.

35. L_ Mémoire â l'appui du projet de Note: $C^{\infty}$-équivalence entre tissus de Veronese et structures bihamiltoniennes, Preprint, 1999.

36. H. W. Turnbull and A. C. Aitken, An introduction to the theory of canonical matrices, Dover Publications Inc., New York, 1961.

37. Alan Weinstein, The local structure of Poisson manifolds, J. Differential Geom. 18 (1983), no. 3, $523-557$.

Department of Mathematics, Ohio State University, 231 W. 18 Ave, Columbus, $\mathrm{OH}, 43210$

E-mail address: ilya@math.ohio-state.edu 